\title{
DEMOGRAPHIC AND CLINICAL CHARACTERISTICS OF INDIVIDUALS' INTENTIONS TO ATTEND CARDIAC REHABILITATION FOLLOWING HOSPITALIZATION FOR HEART FAILURE: A DESCRIPTIVE ANALYSIS
}

By

\author{
Kristen Cunningham \\ BScN, Western University, London, Ontario, 2014 \\ A thesis \\ presented to Ryerson University \\ in partial fulfillment of the \\ requirements for the degree of \\ Master of Nursing \\ In the Program of \\ Nursing \\ Toronto, Ontario, Canada, 2017 \\ (C) Kristen Cunningham 2017
}




\begin{abstract}
AUTHOR'S DECLARATION
I hereby declare that I am the sole author of this thesis. This is a true copy of the thesis, including any required final revisions, as accepted by my examiners.

I authorize Ryerson University to lend this thesis to other institutions or individuals for the purpose of scholarly research.

I further authorize Ryerson University to reproduce this thesis by photocopying or by other means, in total or in part, at the request of other institutions or individuals for the purpose of scholarly research.
\end{abstract}

I understand that my thesis may be made electronically available to the public. 
DEMOGRAPHIC AND CLINICAL CHARACTERSITICS OF INDIVIDUALS' INTENTIONS

TO ATTEND CARDIAC REHABILITATION FOLLOWING HOSPITALIZATION FOR

HEART FAILURE: A DESCRIPTIVE ANALYSIS

\author{
ABSTRACT \\ Kristen Cunningham \\ Master of Nursing Program \\ Ryerson University, Toronto, 2017
}

There is limited literature available that addresses heart failure patient attendance at cardiac rehabilitation centers. This quantitative descriptive pilot study used a convenience sample $(n=30)$ to determine differences in socio-demographic and clinical characteristics and complications among individuals with heart failure who intended or did not intend to attend cardiac rehabilitation six weeks post-discharge. Findings suggest those intending to attend were significantly $(\mathrm{p}<0.05)$ older, unemployed/retired, received an income $>\$ 50,000$, were able to drive, had lower functional classification scores, and experienced fewer complications over six weeks post-discharge. This descriptive pilot study provides an understanding of factors associated with intention to attend cardiac rehabilitation as well as the feasibility of the study design and procedures. Implications focus on strategies to increase potential attendance at cardiac rehabilitation in the heart failure population at the health care provider, organizational and policy levels as well as areas for future research. 


\section{ACKNOWLEDGEMENTS}

I would like to express my sincere thanks to my supervisor, Dr. Suzanne Fredericks for her contributions to this work as well as her expertise and guidance she provided me throughout this process. I would like to extend my gratitude to my thesis committee members Dr. Nancy Walton, Dr. Jennifer Lapum and Helen Kelly whose thoughtful, insightful feedback, and unique perspectives all enhanced my abilities as a writer and to think critically as a researcher.

Special thanks and acknowledgement goes to the patients who willingly participated in this study. Without you, this work would not have been possible and I am grateful for your time.

To my colleagues at Ryerson, you have truly enhanced my graduate experience and I am thankful for the support we were able to provide one another throughout the MN program.

To the Gross family, this journey would not have been the same without you guys and I am so grateful for all of you! Finally, to my family, I cannot thank you enough for providing me with never ending encouragement throughout my academic career, your support in all of my endeavors, and for always keeping me going! I love you dearly. 


\section{DEDICATION}

This thesis is dedicated to my mother for your unwavering love and support over the years.

Thank you for always believing in me and simply being there. I could not have done this without you. Love you lots! 


\section{TABLE OF CONTENTS}

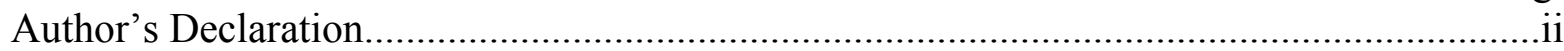

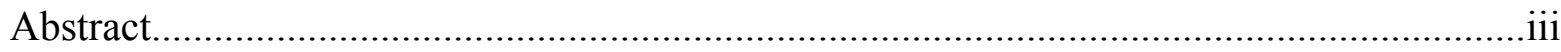

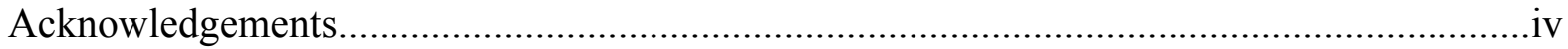

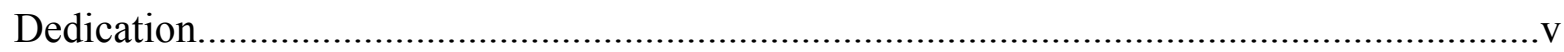

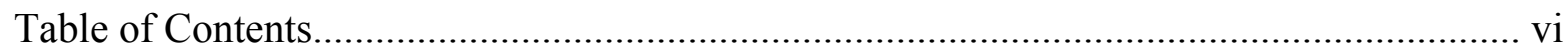

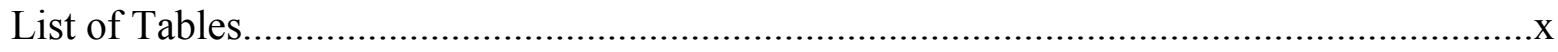

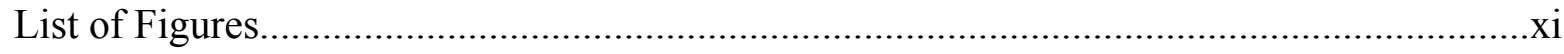

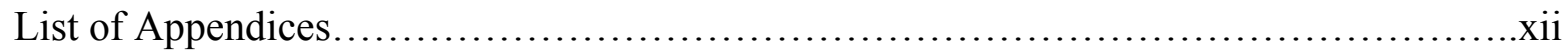

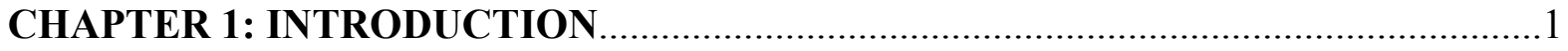

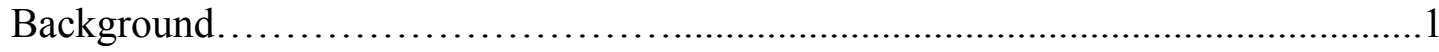

Cardiac Rehabilitation and Heart Failure..................................................................

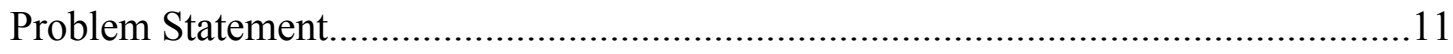

Study Purpose

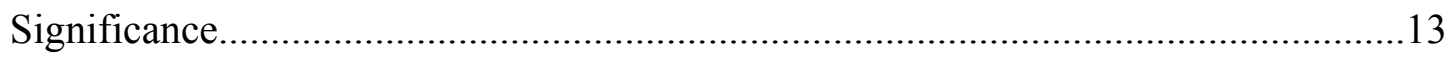

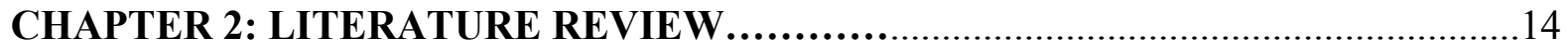

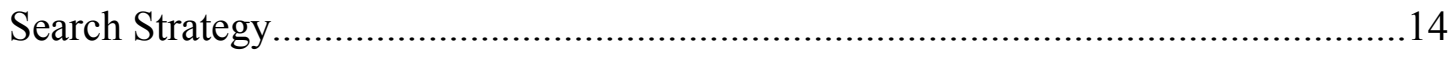

Cardiac Rehabilitation and Heart Failure Overview.................................................16

Cardiac Rehabilitation in Canada...................................................................16

Cardiac Rehabilitation in Heart Failure............................................................17

Individual Determinants of Cardiac Rehabilitation Participation.................................19

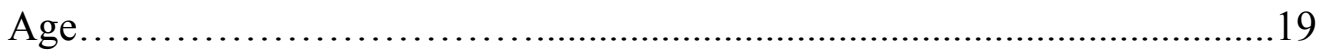

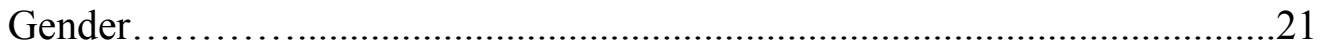


Ethnicity .23

Health Status: Co-Morbidities and Complications........................................24

Perceptions of Illness..............................................................26

Negative Perceptions of Cardiac Rehabilitation...........................................27

Contextual Factors Influencing Participation in Cardiac Rehabilitation......................28

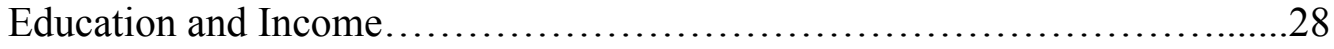

Relationship Status and Support Systems..............................29

Referral, Wait Times, and Health Care Provider Related Factors...............30

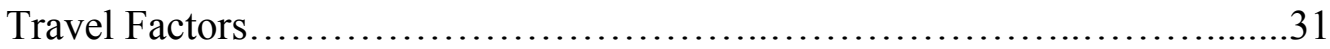

Other Factors........................................................ 31

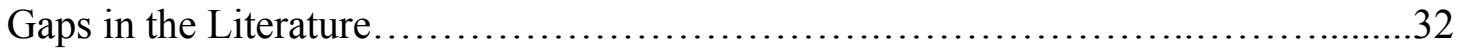

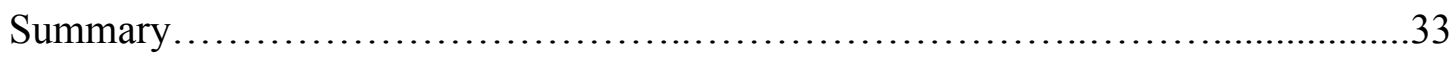

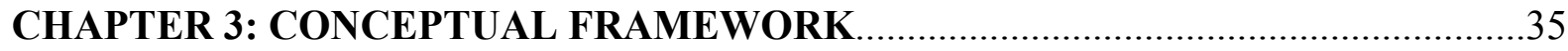

Andersen and Newman's Model of Health Care Service Utilization...........................35

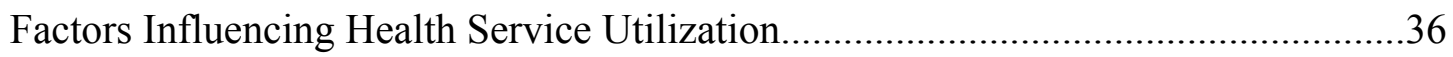

Individual Determinants of Health Service Utilization..........................................

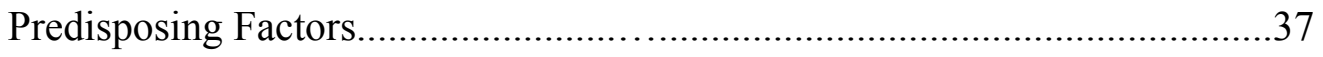

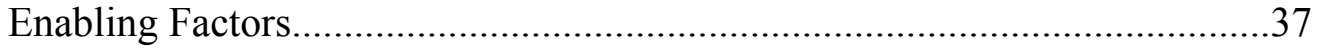

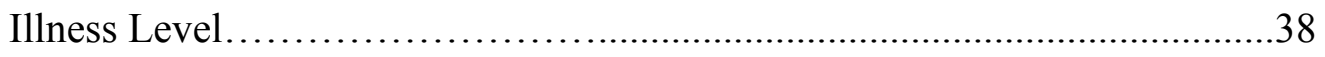

Application to Cardiac Rehabilitation Participation................................... 38

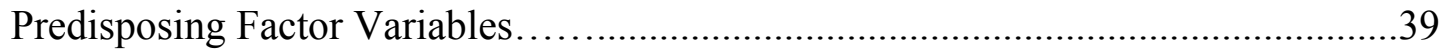

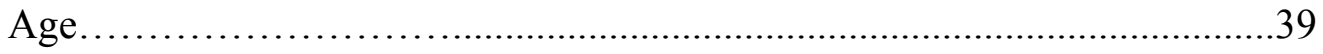

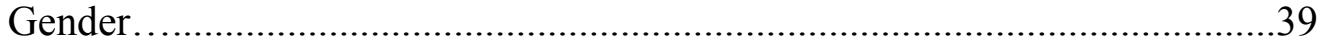




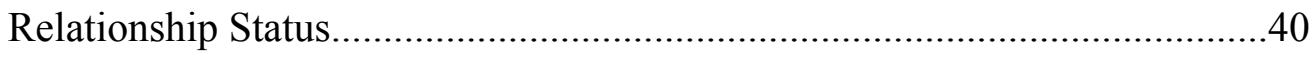

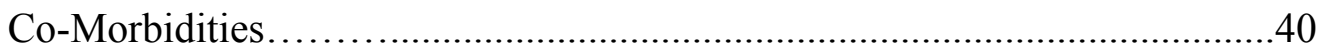

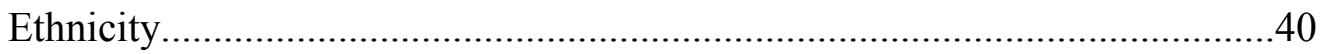

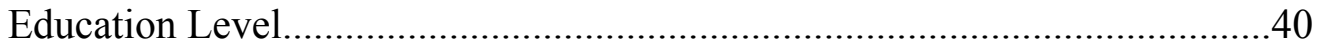

Employment Status..........................................................................4 41

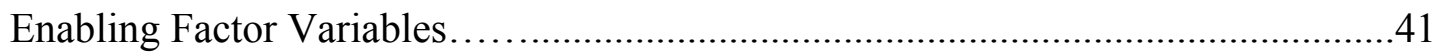

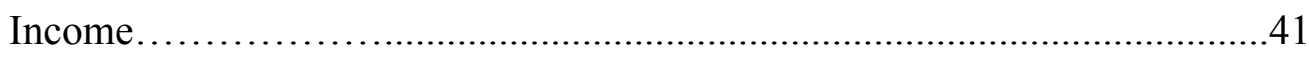

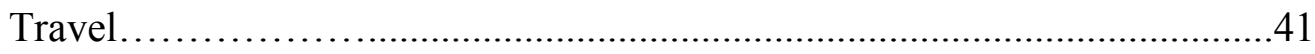

Social Supports................................................................. 41

Living Arrangement..................................................................... 42

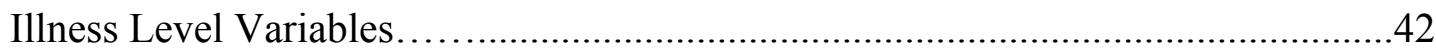

New York Heart Association Classification.............................................42

Heart Failure Related Complications................................................42

Research Questions....................................................................... 43

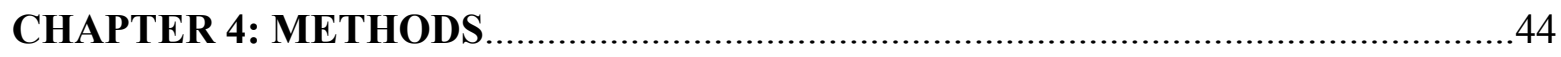

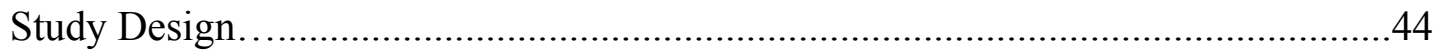

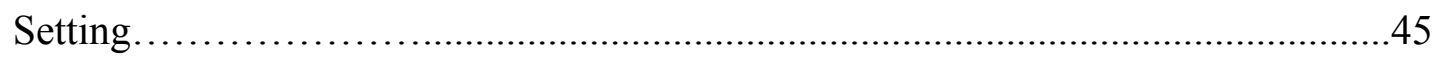

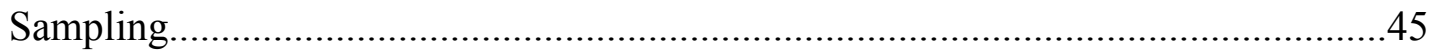

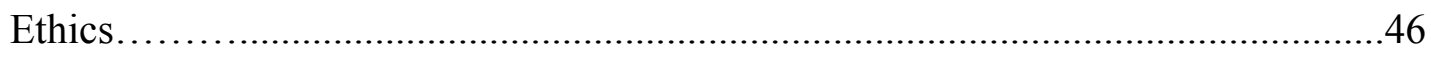

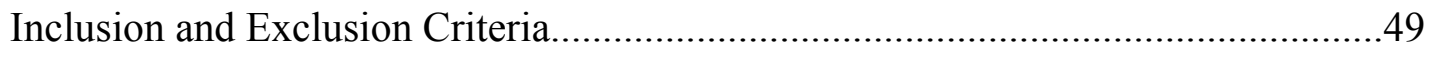

Data Collection Procedures.................................................................................5

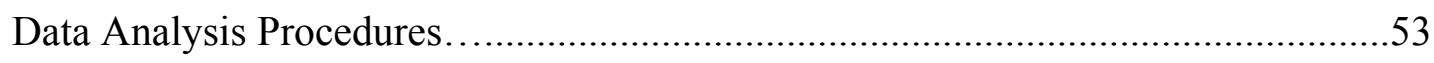

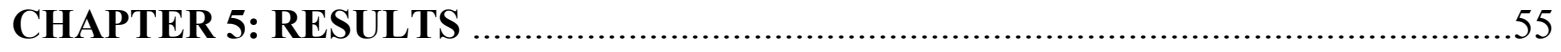


Participation........................................................ 55

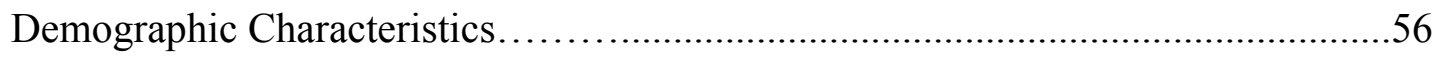

Cardiac Rehabilitation-Related Factors......................................61

Clinical Characteristics................................................61

Total Heart Failure Complications.......................................63

Heart Failure Complications: Weekly and Bi-Weekly Follow-Up.................64

Barriers to Cardiac Rehabilitation Participation.....................................67

CHAPTER 6: DISCUSSION AND IMPLICATIONS ...........................68

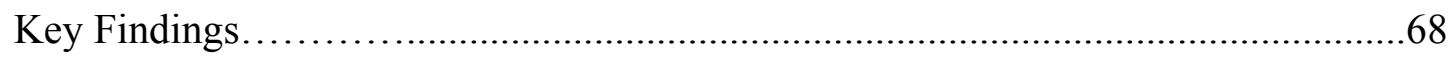

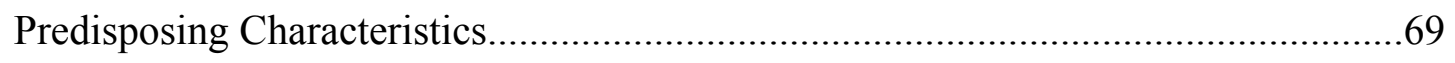

Enabling Factors..................................................... 71

Illness Level........................................................ 73

Rationale for Non Significant Findings.................................... 74

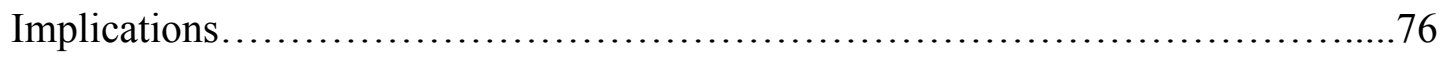

Implications for Practice........................................76

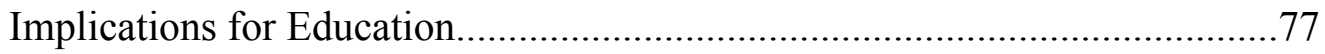

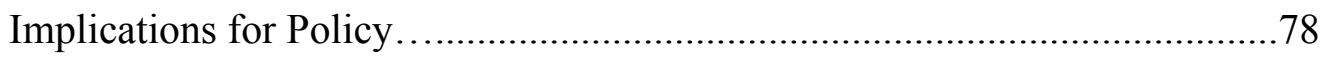

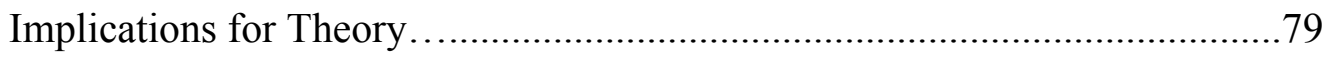

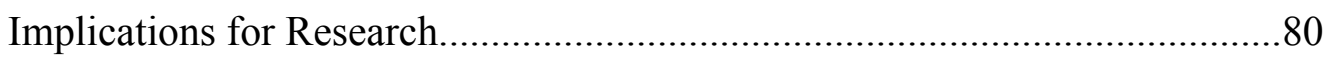

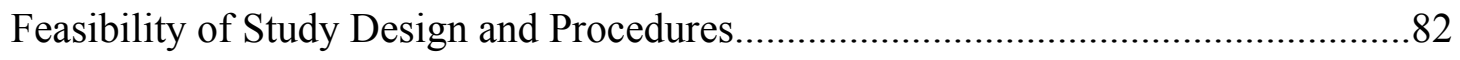

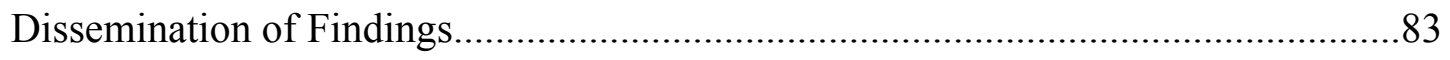

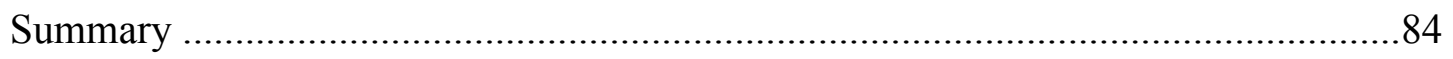

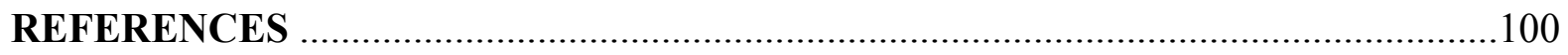




\section{LIST OF TABLES}

Table 1. Baseline Characteristics...........................................58

Table 2. Baseline characteristics (categories collapsed) .........................60

Table 3. Clinical Characteristics and Cardiac Rehabilitation-Related Factors..........62

Table 4. Barriers to Cardiac Rehabilitation Participation...........................67 


\section{LIST OF FIGURES}

Figure 1. Health Service Utilization Model .....................................36

Figure 2. Flow of Participants Through the Study ...............................55

Figure 3. Total Heart Failure-Related Complications $\ldots \ldots \ldots \ldots \ldots \ldots \ldots \ldots \ldots \ldots \ldots \ldots . \ldots 4$

Figure 4. Heart Failure Complications: Week One ..............................65

Figure 5. Heart Failure Complications: Week Three..............................65

Figure 6. Heart Failure Complications: Week Five..............................66

Figure 7. Heart Failure Complications: Week Six...............................66 


\section{LIST OF APPENDICES}

Appendix A: Heart Failure-Related Complications.................................................83

Appendix B: Introduction of Study Script For Circle of Care Members.................84

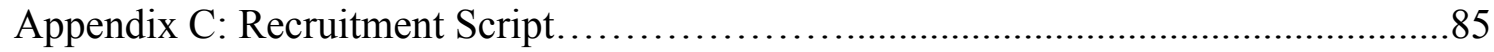

Appendix D: Letter of Information For Patients.................................................... 86

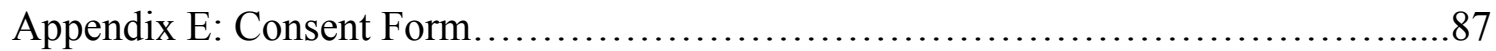

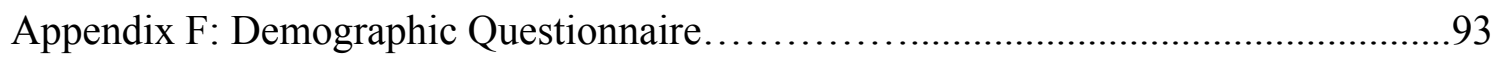

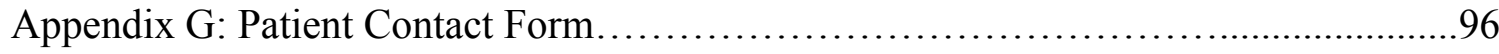

Appendix H: Telephone Call Script: Weeks 1,3,5, 6..............................97 


\section{Chapter One: Introduction}

\section{Background}

Heart failure (HF) is a chronic, progressive condition affecting 23 million individuals globally (Dunlay \& Roger, 2014). It is a severe failure of the heart caused by diseases that damage the structure or function of the heart muscle, thereby reducing the pumping or relaxing action of the heart to meet the body's physiological needs (Heart and Stroke Foundation, 2016). The two leading causes of HF are myocardial infarction and hypertension (Dunlay \& Roger, 2014; Heart and Stroke Foundation, 2016). Significant mortality and morbidity, financial burden, loss of functional capacity and poor quality of life have been linked to HF (Arnold et al., 2006; Bui, Horwich, \& Fonarow, 2011; Johansen, Strauss, Arnold, Moe, \& Liu, 2003; Mozaffarian et al., 2015; Stromberg, 2005).

Over 500,000 Canadians are living with a diagnosis of HF (Ross et al., 2006). On average, approximately 50,000 individuals in Canada and 870,000 patients in the United States (US) are newly diagnosed with HF yearly (Mozaffarian et al., 2015; Ross et al., 2006). These numbers are expected to increase as the population ages and life expectancy continues to increase (Bui et al., 2011). By 2025, HF incidence in Canada is expected to double (Johansen et al., 2003). It is estimated that one in five individuals are at risk for developing this condition over the course of a lifetime (Mozaffarian et al., 2015). The risk of developing HF increases with age (Lee et al., 2004; Mozaffarian et al., 2015). Individuals over the age of 65 account for $85 \%$ of patients hospitalized with HF (Lee et al., 2004). This is of particular significance as the number of Canadians over the age of 65 is projected to double by 2036 (Employment and Social Development Canada, 2015). 
HF is typically characterized by increased shortness of breath (SOB), swelling in peripheral extremities, sudden weight gain of more than five pounds in one week, increased urination at night, lack of appetite, persistent cough or cold symptoms, and decreased physical functioning which may also result in difficulties carrying out activities of daily living (Heart and Stroke Foundation, 2016). Living with HF often requires that patients make significant lifestyle changes in order to manage symptoms after diagnosis including diet, exercise, symptom recognition and management, regular follow-up with health care providers (HCPs) and medication adherence (Heart and Stroke Foundation, 2016).

National strategic plans have been designed to target the following indicators associated with the management of cardiovascular disease (CVD) (including HF): weight loss, heart-healthy diets, smoking cessation, routine health screening for diabetes and cholesterol levels (Smith et al., 2009; Public Health Agency of Canada, 2013). Optimization of drug therapy for controlling hypertension (a primary risk factor for $\mathrm{HF}$ ) has also been shown to reduce the prevalence of $\mathrm{HF}$ (Bui et al., 2011). In addition, outpatient management of CVDs has led to increased multidisciplinary follow-up visits that, in turn, has allowed for earlier assessment and intervention for worsening symptoms (Moser \& Mann, 2002). These advanced treatments have improved patients' rate of survival and life span after a cardiac event (Heart \& Stroke Foundation, 2016). However, individuals are now living longer with damaged heart muscles, increasing their susceptibility for developing HF (Heart and Stroke Foundation, 2016).

Mortality rates for patients with HF remain high, as 50\% of individuals do not survive within the first five years after initial diagnosis (Ross et al., 2006; Parajuli, Ramprasath, Zhabyeyev, Patel, \& Oudit, 2015). HF has been classified as the leading cause of hospitalization, with the highest 30-day readmission rates for patients over the age of 65 compared to all other 
medical conditions (Canadian Institute for Health Information, 2012; Canadian Institute for Health Information, 2015; Desai \& Stevenson, 2012). Approximately 21-24\% of HF patients will be re-admitted within 30 days of discharge and $50 \%$ will be readmitted within six months to one year (Canadian Institute for Health Information, 2012; Desai \& Stevenson, 2012; Johansen et al., 2003; Krumholz et al., 2009). In addition, the average length of stay for HF patients in Canada is twice the length of stay for all-cause admissions (Dai, Walsh, Wielgosz, Gurevich, \& Bancei, 2012). Dai et al., suggest this increased length of stay may be related to the greater number of comorbidities associated with HF compared with other conditions, placing a greater burden on the health care system.

The costs associated with an increasing prevalence of HF constitute a significant burden on the health care system (Bui et al., 2011). The annual direct and indirect costs of HF are estimated to be $\$ 4$ billion in Canada and $\$ 30.7$ billion in the US (Mozaffarian et al., 2015; Parajuli et al., 2015). While there is a lack of more specific Canadian studies, in 2012 the annual acute in-patient cost for HF was Cdn $\$ 166$ million, and an additional Cdn $\$ 37$ million for readmission costs across Ontario (Health Quality Ontario \& Ministry of Health and Long Term Care, 2013). By 2030, costs related to HF are projected to increase by $127 \%$ to US $\$ 69$ billion in the US (Mozaffarian et al., 2015). There are also intangible costs that have a significant impact upon the lives of patients with HF. Loss of functional capacity due to inactivity can contribute to depression in HF patients and decrease overall quality of life (Pina, 2010). Subsequent hospitalizations cause further deconditioning of HF patients due to prolonged periods of bed rest, contributing to morbidity and mortality rates (Pina, 2010).

Despite advances in management and guidelines, adherence to treatment plans remains low following hospital discharge, resulting in avoidable hospital readmissions (Moser et al., 
2012; Paul, 2008; Stromberg, 2005). A number of factors that correspond to individuals experiencing reoccurring HF symptoms and hospital readmissions include: lack of follow-up with primary HCPs after discharge from hospital, insufficient knowledge about self care, and poor adherence to medications and dietary restrictions (Commodore-Mensah \& Dennison Himmelfarb, 2012; Moser et al., 2012; Paul, 2008) compounded by multiple co-morbidities, clinical depression, decreased cognitive functioning, and failure to recognize deterioration in physical functioning (Moser et al., 2012).

One possible reason for the increase in readmission rates may be due to tertiary care institutions not being the ideal setting in which to provide face-to-face HF-related education as many patients experience psychological and emotional stress, physical strain, and anxiety related to the hospitalization and diagnosis experience which may limit their ability to understand and retain education provided to them (Commodore-Mensah \& Dennison Himmelfarb, 2012). Additional limitations of the inpatient setting include lack of time to effectively deliver patient education by HCPs, which may further contribute to ineffective outcomes related to HF patient education interventions. Primary factors contributing to lack of time for HCPs to deliver education in-hospital include system level factors, nursing shortages, faster discharge processes, and lack of reimbursement for additional patient education (Commodore-Mensah \& Dennison Himmelfarb, 2012). Regalbuto et al., (2014) reported 10\% of patients understood the HF-related education instructions prior to discharge. Additional educational support in the outpatient setting, through programs such as cardiac rehabilitation (CR), may be of value to $\mathrm{HF}$ in adhering to their treatment plans once they are discharged from hospital (Dafoe, Arthur, Stokes, Morrin \& Beaton, 2006). 


\section{Cardiac Rehabilitation and Heart Failure}

While there remains no cure for HF, the role of secondary prevention has been one focus for reducing the risk of recurrent events (Dafoe et al., 2006). CR is a secondary prevention intervention that has been identified in the literature as effective for improving post-discharge health, with significant improvements in quality of life, skeletal and respiratory muscle function, while also reducing depressive symptoms and cardiovascular risk factors for patients with all cardiac ailments, including HF (Dafoe et al., 2006; Davidson et al., 2010, Pina, 2010). CR has been found to significantly reduce hospital readmission rates, while reducing mortality rates by approximately $25 \%$ after completion of such programs (Davidson et al., 2010; Grace, Bennett, Ardern \& Clark, 2014; Lavie et al., 2009; O’Connor et al., 2009; Taylor et al., 2004).

Davidson et al., (2010), Davies et al., (2010), and Ghanbari-Firoozabadi et al., (2014) findings suggest $\mathrm{CR}$ provides an ideal setting for outpatient management following hospital discharge for HF complications. They reasoned that providing patients with individualized education on self-management and individual exercise programs in a supervised setting can improve physical functioning. Participants are seen on a regular basis, with access to multidisciplinary services including nurses, physiotherapists, occupational therapists, dieticians, social workers, and physicians, all of whom work with the primary HCP to ensure coordination and continuity of care (Ades et al., 2013). Attendance at CR provides patients with access to HCP's who are well positioned to facilitate early recognition of symptom exacerbation and assist with the appropriate interventions, thus helping to reduce the need for hospital admissions or physician visits (Ades et al., 2013).

CR was introduced initially to target patients' post-myocardial infarct and has been recently introduced as an effective strategy for the HF population (Forman, Sanderson, 
Josephson, Raikhelkar, \& Bittner, 2015). Supervised exercise, a main component of CR, was previously contraindicated in HF due to concerns about exercise being a detriment to the disease process, and as a result HF patients were not referred to CR (Forman et al., 2015; O'Connor, 2006). However, the largest randomized controlled trial (RCT) investigating the safety of exercise in HF, A Controlled Trial Investigating Outcomes of Exercise TraiNing (HF-ACTION) (2009), confirmed the efficacy and safety of exercise while having a statistically significant improvement in patient self-reported health status and reduction in incidence of all-cause mortality and hospitalizations (O'Connor et al., 2009). Findings from the HF-ACTION trial are further supported by meta-analyses demonstrating the safety of structured exercise programs for the HF population, reduced readmission rates, improvements in health-related quality of life (HrQOL), and functional outcomes compared to usual care (Davies et al., 2010; Theou et al., 2011).

CR has evolved from an exercise-only program to a secondary prevention initiative that can identify disease progression and provide necessary interventions to reduce HF exacerbations (Forman et al., 2015). While the actual CR programs for HF vary by institution, key elements in most programs, in addition to exercise training now include: follow-up assessments by multidisciplinary staff, pharmacological therapy, psychological support, and tailored HF education on sodium and fluid restriction, weight monitoring, nutritional counseling, and recognition of signs and symptoms indicating disease progression (Corra et al., 2005; Forman et al., 2015).

The most recent guidelines from the American Heart Association/American College of Cardiology (AHA/ACC) (Yancy et al., 2013), Canadian Cardiovascular Society (CCS) (Moe et al., 2013), and European Society of Cardiology (ESC) (McMurray et al., 2012) recommend 
exercise training in HF should occur through supervised, centre-based rehabilitation programs. It has also been recommended that exercise should be routinely incorporated into care plans for the majority of stable HF patients regardless of age (New York Heart Association functional class IIII) (McMurray et al., 2012; Moe et al, 2013; Yancy et al, 2013). Canada also developed a national strategy, which was released in 2009, to address the growing burden of CVD (Smith et al., 2009). The Canadian Heart Health Strategy and Action Plan (CHHS-AP) identifies CR as an integral component of cardiac care and places emphasis on integrating it into CVD management programs, with early enrollment in such programs for optimal patient outcomes (Smith et al., 2009). Enrollment in structured CR programs is crucial to incorporate into future treatment plans across the continuum of care to address the HF epidemic (Corra et al., 2005).

Despite recommendations outlined in international clinical guidelines (Moe et al., 2013; Yancy et al., 2013; McMurray et al., 2012), the number of individuals who attend CR programs remains low with a participation rate of approximately $30 \%$ among eligible CVD patients in Canada (Grace et al., 2011). Major reasons for these low attendance levels relate to lack of endorsement for $\mathrm{CR}$ by HCPs and lack of understanding by the patient on the role and benefits of CR (Grace et al., 2009). In addition, a lack of public awareness of the benefits of exercise in HF is also a contributing factor to low enrollment (Pina, 2010). A European public awareness survey of exercise in HF found $60 \%$ of the population believed physical activity should be avoided (Pina, 2010). In addition, prior to 2014 the Centers for Medicare \& Medicaid Services (CMS) in the US did not cover CR for HF unless patients had another cardiac diagnosis where coverage existed (Ades, 2015). As a result of lack of coverage, few referrals for HF patients to CR were made prior to 2014 in the US (Ades, 2015). 
Lack of referral is also a contributing factor for the low CR participation rate, as the most recent statistics indicate that, among those who are eligible to attend CR in Canada, only $34 \%$ are referred (Grace et al., 2011). These statistics are inclusive of patients with various cardiovascular conditions, mainly coronary artery bypass surgery, percutaneous coronary intervention, and acute coronary syndrome as these are the top three conditions cited that receive referrals to $\mathrm{CR}$ (Alter, 2012; Grace et al., 2012; Grace et al., 2015). In Ontario, a study examining temporal trends in CR from 1996 to 2010, found referral of inpatient HF patients was low and remained low over the course of the study period (Grace et al., 2015). A recent study assessing temporal trends in CR referral for HF patients in the US, found only one-tenth of eligible HF patients were referred to CR upon hospital discharge (Golwala, 2015). Golwala et al., found patients who were referred to CR were younger, more likely to be male, and had fewer comorbid conditions. Buttery et al., (2014) found $72 \%$ of HF patients aged 65 years and older preferred to attend CR, yet only $21 \%$ were referred. However, individuals over the age of 65 are the largest cohort of individuals living with $\mathrm{HF}$ and represent the population that may benefit most from $\mathrm{CR}$ with access to multidisciplinary services to assist with the increased co-morbidities associated with this age group (Golwala et al., 2015; Grace et al., 2009). Recent meta-analyses of RCTs in CR for HF include studies with significantly younger participants, with the mean age ranging from 43-72 years (Davies et al. 2010). Yet, it is known that many older patients successfully adhere to CR programs after they begin (Grace et al., 2009).

Grace et al., (2009) suggested a lack of referral to CR for the aging population can lead to functional decline and assumptions concerning this age group's ability to engage in CR, lack of physician support or referral and misconceptions around the safety of exercise for patients with HF. In a study exploring barriers to outpatient CR attendance in cardiac outpatients, Grace et al. 
noted older individuals reported experiencing greater barriers than younger individuals. Older patients were less knowledgeable about CR than younger patients, perceived exercise as tiring and painful with uncertainty about the potential health benefits, and felt confident to self-manage their condition at home without CR (Grace et al., 2009).

There are a number of other reasons associated with barriers to CR. Women are also significantly less likely to attend $\mathrm{CR}$, with the most commons barriers identified as lack of transportation, financial difficulties, and lack of emotional or social supports (Dunlay et al., 2009; Mampuya, 2012; Taylor, Wilson, \& Sharp, 2011). In addition, other studies have identified a lack of participation in some racial minorities (Grace, Parsons, Heiss, \& Bacon, 2015; Mampuya, et al., 2012) due to lack of accessibility to programs, referral, and insurance coverage in the US (Mampuya, 2012). Statistically significant differences between socioeconomic status and attendance at CR have also been found (Mampuya, 2012; Taylor et al., 2011). Unemployment status and inability to drive have been cited as factors for non-attendance for CR (Dunlay et al., 2009).

Additionally, long wait times also have a negative impact on attendance in CR programs (Russell et al., 2011). In 2005, the CCS Access to Working Care Group developed recommendations for wait times to CR. The CCS Access to Working Care Group identified 30days as the benchmark and 60 days as an acceptable wait time between referral and attendance in CR programs for the HF patient population, among other cardiovascular conditions (Dafoe et al., 2006). However, research demonstrated this benchmark wait time was not being met in Canada (Grace et al., 2015). In a study using data from the Canadian CR registry, average wait time from referral to intake was 68 days +/- (standard deviation) of 64 days among the 12 out of 154 known CR programs in Canada participating in the registry (Grace et al., 2015). Grace et al. reported 
that younger, white, married males are more likely to be referred and attend Canadian CR programs. At the time of CR program admission: $86 \%$ of patients travelled 30 minutes or less to CR, $62 \%$ attained high school education as the highest level of education, $70 \%$ identified as White/Caucasian, and were 66.3 +/- 11.5 (standard deviation) years of age (Grace et al., 2015). In a smaller study in Ontario, patients reported a median wait time of 42 days from hospital discharge to uptake of CR (Grace et al., 2012). Data from The Canadian Cardiac Network of Ontario CR Pilot Project, undertaken in 2002, found the average wait time from a cardiac event to intake in CR was 99 days, and the median time was 70 days (Dafoe et al., 2006). Average time from actual referral to $\mathrm{CR}$ until intake in $\mathrm{CR}$ was 40 days, and median time from referral to intake was 31 days (Dafoe et al., 2006).

Automatic referral processes have been introduced in Canada to ensure all patients who are eligible for CR are referred, regardless of socio-demographic characteristics (Grace et al., 2015; Smith, Harkness, \& Arthur, 2006). Automatic referrals have been found to be related to a $23-28 \%$ increase in CR enrollment compared to other methods of recruitment reflected in the literature (Grace et al., 2004). In addition, automatic referral processes have been beneficial in both reducing wait times for CR and increasing CR attendance rates (Dunlay et al., 2009; Grace et al., 2004).

The majority of data available on CR attendance wait times and socio-demographic characteristics of patients who attend CR in National databases and the Canadian CR registry were largely based on specific cardiac populations (Grace et al., 2015). The top three cardiac populations discussed in the CR literature are: post myocardial infarction patients, patients post angiogram or cardiac catheterization and coronary artery bypass graft (CABG) surgical patients (Grace et al., 2015). Limited data were available on the demographic and clinical predictors of 
CR attendance specific to the HF population. This lack of data can be attributed, in part, due to the fact that $\mathrm{CR}$ for HF is a fairly new secondary prevention intervention in which the safety and safety and efficacy of exercise has been demonstrated in the HF population (O'Connor, 2009).

In summary, a number of significant changes have been made to incorporate $\mathrm{CR}$ as a necessary component of the outpatient management for HF, including: introduction of the automatic referral process for $\mathrm{CR}$ for cardiac conditions, the development of national guidelines for the safety and efficacy of CR in HF populations, and the uptake in use of evidence supporting the improved clinical outcomes for HF patients after attendance in CR. Despite these changes, the low uptake of such programs by those referred warrants further research.

\section{Problem Statement}

The CR literature as it pertains to HF, has mainly focused on outcomes of patients who enroll and attend such programs, and specifically on patient outcomes related to exercise capacity, readmission rates, all-cause mortality and HrQOL for patients who attend (GhanbariFiroozabadi et al., 2014; Lewinter et al., 2014; Pina, 2010). Presently, there is insufficient data on HF patient attendance. As well, there does not appear to be any study in the current literature examining the demographic and clinical characteristics of HF patients who are referred for CR. In order for CR programs to be relevant to all referred patients, it is important to identify if there are any distinguishing characteristics among patients with $\mathrm{HF}$ who intend to attend $\mathrm{CR}$ programs and those who do not intend to attend these programs.

Timely access to cardiac care in the outpatient setting is necessary to ensure postdischarge health and optimal patient outcomes. There is limited current research exploring health-related complications experienced by individuals with HF awaiting intake into CR and whether timely access decreases health-related complications compared with delayed referral or 
intake (Moe et al., 2013). Patients with HF have a high risk of recurrent events and exacerbations post-discharge from an acute hospitalization (Moser et al., 2012; Paul, 2008; Stromberg, 2005). Even with the recommended benchmark wait time of 30 days for intake into CR not currently being met, there has been very little research into the potential complications experienced by HF patients while awaiting intake into CR programs (Grace et al., 2015). As HF accounts for the highest 30-day admission rates in Canada (Canadian Institute for Health Information, 2015), further research is warranted to explore patient health conditions within this important time frame.

\section{Study Purpose}

The purpose of this descriptive pilot study was:

1. To determine the difference in socio-demographic and clinical characteristics among individuals with $\mathrm{HF}$ who intend to attend $\mathrm{CR}$ six weeks following hospital discharge and those who do not intend to attend CR.

2. To determine the difference in the type and number of HF-complications among individuals who intend to attend CR six weeks following hospital discharge and those who do not intend to attend CR.

3. To examine the feasibility of the study design and procedures.

The specific characteristics that were considered are: age, gender, ethnicity, education level, employment status, income level, relationship status, family supports, living arrangement, distance from CR facility, transportation access, New York Heart Association classification (IIV), and number of co-morbid conditions. 


\section{Significance}

This study contributed to the current body of Canadian and international knowledge related to $\mathrm{CR}$ and the HF population by highlighting the socio-demographic and clinical characteristics of all patients with HF who are referred to CR. As well, a preliminary body of literature that addresses HF-related complications prior to engagement in $\mathrm{CR}$, and a possible relationship between complications and attendance in CR was generated.

The findings from this study may benefit the management of HF in the outpatient setting. Findings may support the development of interventions and strategies targeted towards increasing attendance at CR among individuals with demographic and clinical characteristics associated with participants who do not intend to attend CR. The study findings on the number and type of complications experienced by the HF patient population while waiting for CR intake may contribute relevant information to help think about what types of services should be more readily available to meet these patients' needs while waiting to begin CR programs. For example, it may be the case that access to more home care supports or earlier intake into the CR programs may be recommended, based on the data collected regarding complications of this population while patients await CR intake. Finally this study may help inform the development of strategies that may address inequitable access and improve wait times for CR in the HF patient population. 


\section{Chapter 2: Literature Review}

This chapter provides an overview of the existing literature on socio-demographic characteristics associated with patient enrollment, attendance, and adherence with CR, as well as contextual factors influencing participation in CR programs. Gaps in the existing literature are identified. The outcome of this literature review provides support for the study purpose and research questions.

\section{Search Strategy}

A search of the scholarly literature was conducted to identify studies that included sociodemographic characteristics and contextual factors influencing patient enrollment (intention to enroll or attendance at initial intake visit), attendance (attending at least one session) and adherence (completion of program, attending all sessions which varied across studies) to CR programs. Cumulative Index Nursing and Allied Health Literature (CINAHL), Ovid Medline, EMBASE, and Google scholar were searched for articles relevant to this study. The BOOLEAN operators "AND" and "OR" were used to combine key words and the delimiter '*' was used to search for multiple characters. The following keywords and controlled vocabulary were searched in combination: CVDs, cardiovascular surgical procedures, HF, cardiac, heart, coronary, rehabilitation, rehabilitation nursing, $\mathrm{CR}$, rehabilitation centers, communication barriers, health services accessibility, socioeconomic factors, rural populations, vulnerable populations, barriers, compliance, dropouts, participation, treatment refusal, disparities, health care delivery, health care disparity, health beliefs, patient satisfaction, demographic, epidemiologic factors, sex, age, population groups, residence characteristics, family characteristics, marital, employment, economic, socioeconomic, education. 
The search was intentionally kept broad to include cardiac conditions in addition to HF, due to limited literature specific to HF and participation in CR. Similarly, outcomes of interest were intentionally kept broad and included research related to CR participation from various medical cardiac diagnoses in order to provide insight into what also may be reflective of characteristics of the HF population. Other medical cardiac conditions included myocardial infarction (MI), coronary artery disease (CAD), coronary heart disease (CHD), acute coronary syndrome (ACS), ischemic heart disease (IHD), angina, and percutaneous coronary intervention (PCI), angiography, or coronary artery bypass graft surgery (CABG). Findings pertaining to the key socio-demographic characteristics of interest in this study were extracted from the articles.

Given the breadth of conditions that could be included, the floating subheading for rehabilitation "rh" was used in Medline and EMBASE to focus the search. The search was further limited to English, full-text, peer-reviewed articles from 2009-2016 for demographic characteristics of cardiac patient attendance at CR, as CR safety in the HF population has only been demonstrated in the literature since 2009, and from 2006-2016 for barriers to attendance at CR. Due to limited literature on HF-specific patient attendance at CR, reference lists of articles were also manually searched for additional relevant empirical findings. Both quantitative and qualitative articles were reviewed along with systematic reviews and meta-analyses. Articles were excluded if they were duplicates or articles in which the population of interest was focused solely on post-CABG, patients awaiting heart transplant or with left-ventricular assistive devices as these patient populations have different recovery needs or physical limitations compared to medical cardiac patients, which may influence factors to attendance at $\mathrm{CR}$. A review of the scholarly literature revealed several socio-demographic factors associated with patient attendance at $\mathrm{CR}$ as well as individual and contextual barriers that may impede attendance at 
such programs. A total of 789 articles were accessed from the searches: EMBASE (200 results), MEDLINE (420), CINAHL (159), Google Scholar (10). Through title and abstract screening and scanning references of relevant articles, the search results were narrowed to 47 articles (1 qualitative, 46 quantitative) and 4 systematic reviews (including qualitative, quantitative and mixed-methods findings)

\section{Cardiac Rehabilitation and Heart Failure Overview}

\section{Cardiac Rehabilitation in Canada}

CR is recognized as standard of care in Canada with referral and participation strongly supported by Canadian Cardiovascular Society practice guidelines for the HF population (Moe et al., 2013), as well as internationally supported by the American Heart Association (Mozaffarian, 2013), and European Society of Cardiology (Zeiher, 2012). Although the delivery of CR programs may vary slightly among Canadian centres, all programs are expected to include 10 core components as outlined in the Canadian Guidelines for CR and CVD Prevention: "systematic patient referrals to CR, intake assessments, health behavior interventions and risk factor modification, adaptations of program models to improve accessibility especially for underserved populations, development of self-management techniques based around individualized assessment, problem-solving, goal-setting, and follow-up, exercise training leisure-time activities, outcomes assessment and performance measurement, continuous quality improvement programs, and professional development programs" (Grace et al., 2014, p.531-532).

Approximately $70 \%$ of CR programs in Canada are site-based in hospitals with an average of 2-3 sessions per week over a median of 5 months (Grace et al., 2014). 


\section{Cardiac Rehabilitation in Heart Failure}

CR in Canada was created during the 1980's for individuals following MI, however limited research was available related to HF patient participation in these programs until recently. The safety and efficacy of exercise in the HF population was confirmed in 2009, prior to which exercise was contraindicated (O'Connor, 2009). Since findings from HF-ACTION trial confirmed the benefits of exercise in the HF population, multiple studies have investigated the impact of CR exercise and education programs on clinical outcomes such as mortality, morbidity, functional capacity, readmission rates and quality of life (Davidson, et al., 2010 Davies et al., 2010; Taylor et al., 2014).

Investigating the benefits of exercise training in the HF patient population, the HFACTION trial found no significant reductions in all-cause mortality or hospitalization rates in unadjusted analyses (O’Connor, 2009). However, after adjustment of prognostic variables, exercise training was found to reduce the incidence of all-cause hospitalizations and mortality by $11 \%$ in patients randomized to the exercise-training group (O’Connor, 2009). Conversely, a recent Cochrane systematic review and meta-analyses of 19 RCT's reported no significant reductions in all cause hospitalizations or mortality when comparing exercised-based rehabilitation to usual care in patients with $\mathrm{HF}$, however a $28 \%$ reduction in HF-related hospitalizations was reported (Davies et al., 2010).

Other studies reported significant reductions in mortality among patients with HF who attended CR compared to those who did not attend (Belardinelli, Gergiou, Cianci, \& Purcaro, 2012; Davidson et al., 2010; Hammill, Curits, Schulman, \& Whellan, 2010) as well as a reduction in all-cause readmissions (Belardinelli et al., 2012; Davidson, 2010). Multiple studies have demonstrated improvements in HRQoL in the HF and general cardiac population after 
attending CR as compared with CR non-attendees (Belardinelli et al., 2012; Davidson et al., 2010; Davies et al., 2010; Leung et al., 2011).

Additional benefits of CR identified in the literature include significant improvements in self-reported health status (Flynn et al., 2009), functional capacity (Davidson et al., 2010; Flynn et al., 2009; Savage et al., 2011; Taylor et al., 2014), New York Heart Association (NYHA) functional classification (Flynn et al., 2009), cardiac function (Alves et al., 2012; Davidson et al., 2010), HF-knowledge and performance of self-care behaviours that includes regular weighing, medication adherence, smoking cessation and sodium restrictions (Baranson et al., 2011). In particular, Davidson et al., conducted a RCT that included 105 patients diagnosed and living with HF who were randomized to receive 12 weeks of formal exercise training and selfmanagement in CR. A comparison group was used as the control in which they received usual regular follow-up care from their primary physician. At the 1-year follow-up of patients, Davidson et al., found significant reductions in all-cause mortality and cardiac-related readmissions. Similarly, Belardinelli et al., (2010) conducted a RCT of 123 patients diagnosed with HF who were randomized to either a formal exercise training group or control group that did not receive any formal exercise training. At the 10-year follow up, Belardinelli et al., found significant reductions in HRQoL, all-cause hospital readmission and cardiac mortality in patients randomized to the the exercise-training arm.

Findings from these studies suggest there are significant sustained, long-term benefits of CR both in regard to the formal exercise and self-management education components. The data from the literature supports CR as beneficial not only in improvements in exercise capacity but in the overall management of HF with improved clinical outcomes as compared with usual care. 


\section{Individual Determinants of CR Participation}

Age

Age is a common factor extensively cited in the literature as it relates to CR participation, with older age being a significant predictor of non-attendance (Dunlay et al., 2009; Hutchinson, Meyer, \& Marshall, 2015; Leung et al., 2011; Turk-Adawi, Oldridge, Tarima, Stason, \& Shepard, 2014). Although HF primarily affects individuals over the age of 65 (Bui et al., 2011) studies have found individuals in this age group are least likely to access CR programs (Hutchinson, 2015; Martin et al., 2012; Turk-Adawi et al., 2014).

In a prospective study evaluating CR participation rates after hospitalization for HF, acute coronary syndrome (ACS), or cardiac surgery, Pardaens, Kerckhove, Willems, Moerman and De Sutter (2013) found participants were significantly younger than those who did not attend (mean age of 65 years versus 79 years respectively). Another prospective study examining sociodemographic barriers to CR adherence for individualized hospital for MI also found significant differences between the mean ages of CR participants (62.0 years) versus non-participants (71.1 years) (Dunlay et al., 2009). Several additional studies inclusive of various cardiac populations (post-CABG, valve replacement, CAD, angina, MI, HF or a combination) have also found younger age, specifically less than 65 years of age (Leung et al., 2011; Turk-Adawi et al., 2014) and 75 years of age (Hutchinson, 2015; Martin et al., 2012), to be significantly and positively associated with $\mathrm{CR}$ attendance. In an exploratory descriptive study analyzing demographic and cardiac characteristics of patients diagnosed with CVD who attended or did not attend CR, McDonall, Botti, Redley, and Wood (2013) found increased age was significantly associated with decreased CR participation. For every 1-year age increase, CR participation decreased by 3\% (McDonall et al., 2013). Conversely, Blair et al. (2014) found no differences in age among 
CR attendees versus non-attendees, although the specific cardiac diagnoses of study participants were not included. Similar findings in a systematic review by Murray, Craigs, Hill, Honey and House (2012) exploring influences predicting uptake and completion of CR in patients with CVD found no difference in age relating to CR uptake. However older age was associated with higher rates of completion once enrolled (Murray et al., 2012). Another study by Anderson and Emery (2014) also found older cardiac participants (PCI, MI, CAD, or CABG) were significantly more likely to attend a greater number of CR sessions compared with younger participants after CR enrollment, however the number of sessions was not specified (age of participants ranged from 31 to 84 years).

Several reasons for non-participation in CR by older individuals have been identified in the literature including lack of referrals or CR endorsement from HCPs for patients who are greater than 65 years old (Grace et al., 2009). Hutchinson et al., (2015) explored factors influencing outpatient CR attendance in patients with ACS and found that individuals over 75 years of age were significantly less likely to receive a referral to CR by their HCP. Other factors related to non-participation include frailty and increased functional decline associated with aging in general, as well as older individual's personal choice to not attend (Mckee et al., 2014). Factors reported as barriers to $\mathrm{CR}$ by older individuals include the perception of being too old to attend (McDonall et al., 2013), or the belief that CR would not make a difference to one's condition (Jackson, McKinstry, Gregory, \& Amos, 2012). Other barriers identified by Grace et al., (2009) in their study exploring barriers to CR in older individuals included negative perceptions of exercise, having co-morbidities, and misconceptions regarding the appropriateness of CR for older individuals or purpose of the programs. 
In the largest study exploring exercise rehabilitation in the HF population, the HFACTION trial, the average age of study participants was 59 years (O'Connor et al., 2009), with less than $5 \%$ of participants over the age of 70 (Forman et al., 2009). Similarly, in their systematic review Davies et al., (2010) found fewer older individuals with HF were randomized to $\mathrm{CR}$ intervention groups with mean ages ranging from 43 to 72 years.

\section{Gender}

Gender is another key variable found to have a significant effect on CR attendance (Taylor et al., 2011). Multiple studies have demonstrated gender differences in CR attendance rates with females significantly less likely to attend (Armstrong et al., 2014; Doll et al., 2015; Dunlay, 2009; Martin et al., 2015; McKee et al., 2014). Females are also underrepresented in studies exploring attendance at CR programs (Hutchinson et al., 2015; Leung et al., 2011, Piotrowicz et al., 2010). Only one study was found which explored gender differences among CR attendees versus non-attendees in the HF population (Pardaens et al., 2013). Findings from Pardeans et al., revealed females were significantly less likely to attend. One study was found which reported patient characteristics specific to the HF population, in which the researchers sought to evaluate the efficacy of a home-based CR program compared with standard outpatient CR programs by randomizing participants to either program (Piotrowicz et al., 2010).

Descriptive statistics revealed only $5 \%$ of participants in the standard CR program were female. McDonall et al., (2013) found males with CVD were 2.5 times more likely to attend CR than females. Dunlay et al., also found significant differences in attendance rates after referral with $71.3 \%$ of males attending compared with $56.3 \%$ of female patients. Among women who do attend CR, they are more likely to be older and report a higher number of barriers to attendance than men, specifically lower socioeconomic status, difficulties with travel and transportation and 
lack of family or friend supports (Taylor et al., 2011). McKee et al., examined factors influencing patients' intention to attend $\mathrm{CR}$, and found a higher percentage of males (85.9\%) intended to enroll in CR compared with females (78.7\%). Once enrolled, Doll et al., found males were more likely to complete a greater number of CR sessions ( $>26$ sessions) than females (1-25 sessions) in their retrospective review of patient adherence to CR after hospitalization for MI. Similar retrospective findings of patients with documented CAD by Armstrong et al., revealed a greater percentage of females dropped out of CR than males ( $21 \%$ and $31 \%$ respectively). Additional prospective findings by Martin et al., also identified less (38.4\%) women who were diagnosed with $\mathrm{CAD}$ and referred to $\mathrm{CR}$ completed the program compared to men (52.1\%). Yohannes, Yalfani, Doherty, and Bundy (2007) also found women were 5 times more likely to drop out of CR than men. In their study examining the effects of depression and anxiety on completion of $\mathrm{CR}$ in patients with $\mathrm{HF}$, MI, angina, or CABG, McGrady, McGinnis, Badenhop, Bentle and Raiput (2009), reported higher dropout rates in females compared with male participants (55\% of women compared with $48 \%$ of men), although it was not reported whether these findings were statistically significant. Conversely, three studies found no statistically significant differences in gender among CR attendees versus non-attendees (Anderson \& Emery, 2014; Blair et al., 2014; Turk-Adawi et al., 2014) while one study found women with CAD were more likely to complete CR than men (Sarrafzadegan et al., 2007).

In Canada, the most recent statistics suggest females are $36 \%$ less likely to elect to enroll in CR than males (Heart and Stroke Foundation, 2016). However, this may be due to lower CR referral rates for women. Several additional factors have been highlighted in a qualitative metasynthesis by Clark et al., (2013) exploring influences to participation in CR after referral and initial attendance. Clarke et al., found female participants viewed CR as a stressor on their family 
life, had less time to commit to $\mathrm{CR}$, viewed $\mathrm{CR}$ as less suitable for women and were less likely to receive family support or ask family members for transportation assistance. Gender roles were also identified as a barrier to $\mathrm{CR}$ participation as women identified attendance at $\mathrm{CR}$ as interfering with traditional roles of child care, housework and family life (Clarke et al., 2013; Jackson et al., 2012).

\section{Ethnicity}

Limited information related to ethnicity and CR participation was available in the literature, as this variable was not consistently reported in the studies included in this review. Racial minorities appear to be underrepresented in the CR literature compared to Caucasian participants (Leung et al., 2011; McGrady et al., 2009; Murray, Murphy, Clements, Brown, \& Connolly, 2013; Parashar et al., 2012). In a study by Leung et al., comparing HRQoL scores for non-CR participants versus participants in a CR program, over $80 \%$ of participants in all groups were Caucasian. Parashar et al., reported similar sample characteristics in their study examining predictors on enrollment in CR among patients referred after acute MI, with Caucasian participants accounting for $92.1 \%$ of the sample in the CR participant group and $81.0 \%$ of nonparticipants. Parashar et al., reported African American participants were less likely to attend CR at 1 month and 6 month follow-up periods. Anderson and Emery (2014) also found African American patients to be significantly less likely to adhere to CR after enrollment compared to Caucasian participants. Findings from one study revealed ethnicity (White, Arab, Asian, Black and Other) did not influence adherence to CR (Murray et al., 2013). Furthermore, Anderson and Emery also found African American patients scored higher on irrational health belief scores, possibly indicating cultural factors may influence beliefs and attitudes towards CR. Findings from a systematic review by Clarke et al., (2013) support that cultural factors may have an 
influence upon decisions to attend or not attend CR. They found that CR participants who identified as being from ethnic minorities viewed CR as lacking cultural sensitivity, lacking inclusivity in terms of languages and clothing preferences during exercise.

Lack of referrals may also be another reason related to decreased participation among ethnic minorities, despite some populations having higher reported rates of CVD compared to Caucasian populations (Gregory, LaVeist, \& Simpson, 2006; Mochari, Lee, Kligfield, \& Mosca, 2006). In their evaluation of physician referrals to CR, Mochari et al. found Caucasians were more likely to be referred and encouraged to attend CR than African American and Latino women. In assessing racial disparities in access to CR, Gregory, LaVeist, and Simpson (2006) found race to be independently associated with CR participation, and found that Caucasian patients were more likely to be referred to CR than African American patients.

\section{Health Status: Co-Morbidities and Complications}

Health status has also been cited as a key variable in predicting CR participation. Doll et al., (2015) revealed post-MI patients who enrolled in CR after referral were significantly less likely to complete the program if they had a higher prevalence of co-morbidities, of which prior HF, PCI or CABG were significant predictors of earlier dropout. A study by Leung et al., (2011), found patients who reported an increased number of co-morbid conditions were also significantly less likely to attend CR, however CAD patients with a history of HF, CABG or PCI were significantly more likely to attend CR. Other studies have also found a higher prevalence of comorbidities, including HF, to be negatively associated with CR attendance (Martin et al., 2012; Parashar et al., 2012). In examining patients and organizational factors associated with CR enrollment, Turk-Adawi et al., (2012) found patients were significantly less likely to enroll if they had a diagnosis of HF, AMI, or pervious CABG compared with other cardiac conditions. In 
another study examining the effects of depression and anxiety on completion of CR in patients with $\mathrm{HF}$, MI, angina, or CABG findings revealed only $50 \%$ of entering patients completed $\mathrm{CR}$, with the dropout rates highest in the HF population (McGrady et al., 2009). In their qualitative descriptive study exploring barriers to CR participation Jackson et al., (2012) found nonparticipants felt the prevalence of several health conditions limited their ability to regain better health and CR would not make a difference in their health status. One study found intention to attend CR was not influenced by co-morbidities or smoking status (Mckee et al., 2014) and another found no difference in depressive symptoms among participants versus non-participants (Parashar et al., 2014).

Other co-morbidities found to be negatively associated with CR attendance include the prevalence of diabetes mellitus (Armstrong et al., 2014; Dunlay et al., 2009; Turk-Adawi et al., 2014; Weingarten et al., 2014) and being an active smoker (Doll et al., 2015; Parashar et al., 2014; Sarrafzadegan et al., 2007; Taylor et al., 2011; Turk-Adawi et al., 2014; Weingarten et al., 2014). In their retrospective cohort study, Armstrong et al., found diabetes to have a significant impact upon attendance rates in patients enrolled in $\mathrm{CR}$ with a diagnosis of CAD, with $84.9 \%$ of non-diabetics completing the 12 -week program compared with $79.6 \%$ of diabetic patients. Diabetic patients with CAD also had a significantly higher number of co-morbidities, including HF. History of depression (Turk-Adawi et al., 2014) or higher depressive symptoms (Leung et al., 2014; McGrady et al., 2009 McKee et al., 2014; Taylor et al., 2011) have also been cited as factors predicting poor participation in CR. In their study examining the effects of depression and anxiety on completion of CR in patients with HF, MI, angina, or CABG, McGrady et al., found dropouts had significant higher baseline depression and anxiety scores than those who completed the program. 
Conversely three studies found no association between depressive symptoms and CR attendance (Parashar et al., 2014) or adherence to CR once enrolled (Anderson \& Emery, 2014; Murray et al., 2013). It is important to note that two of these studies only examined adherence to CR once enrolled and did not examine attendance versus non-attendance, suggesting that depressive symptoms may have an impact an individual's decision to attend CR, however may not have an influence upon adherence to CR programs once enrolled (Anderson \& Emery, 2014; Murray et al., 2013).

\section{Perceptions of Illness}

Patient beliefs and attitudes towards CR are also important factors to consider when exploring influences on CR attendance. In their study examining knowledge and belief risk factor information among CHD patients (MI, $\mathrm{CABG}$, and $\mathrm{PCI}$ ) who declined participation in $\mathrm{CR}$, Almerud-Osterburg et al., (2011) found non-attendees had lower levels of knowledge about risk factors and management of CHD. Among non-attendees, Almerud-Osteburg et al., found those who reported higher levels of education obtained more information on risk factors and management of CHD. In their study examining socio-demographic factors, illness perception and social isolation on CR attendance, Blair et al., (2014) found non-attendees had more negative perceptions about their cardiac illness than attendees. In addition, non-attendees were more likely to attribute the cause of their illness to non-modifiable risk factors, suggesting they may not view $\mathrm{CR}$ as having any benefit to improving their health. A recent systematic review and metasynthesis by Clark et al., (2013) on CHD patient barriers to participation in CR, found patient identity as a major theme: this refers to the struggle some patients experience as they try to accept their diagnosis as part of their self-identity, subsequently not recognizing the need for CR services. In addition Clark et al., identified that non-participants differentiated themselves from 
those who they considered to be a more typical 'type of patient' they considered to enroll in CR program. They tended to assume that a typical CR attendee would be older, more unwell and more unfit. As they differentiated themselves from their idea of a typical attendee, these individuals viewed $\mathrm{CR}$ as less beneficial and subsequently, tended to choose to not attend.

Illness cognition has also been examined as a predictor of CR participation. This term refers to the way individuals' perceive their current situation as it relates to their illness level and how they cope with it (Reges et al., 2013). Results from a descriptive study by Reges et al., revealed individuals who perceived exercise as beneficial to overall health perceptions and those with a higher sense of personal control were more likely to attend CR. Participants with a greater perceived severity of CHD or those who felt they were likely to have an additional cardiac event, were less likely to attend $\mathrm{CR}$, with a possible explanation as participants perceiving exercise as harmful to their health (Reges et al., 2013). These results are consistent with findings from other systematic reviews, which found low-self efficacy and perceptions of less control over their illness to be predictive of non-attendance at CR (Murray et al., 2012). Anderson and Emery's (2014) study revealed health beliefs predicted CR attendance, with individuals who had higher irrational health belief scores, which were defined in this study as having beliefs not grounded in medical science, less likely to adhere to CR once enrolled.

\section{Negative Perceptions of CR}

Negative experiences or views towards CR programs have also been cited as having an impact upon an individual's decision to attend CR after referral. Among CHD patients, Clark et al., (2013) found that HCPs provide inconsistent information related to CR and that approaches to endorsing $\mathrm{CR}$ are often experienced by participants as being overly aggressive, forceful, and lacking in cultural sensitivity. Similar findings from a study exploring factors influencing 
attendance at CR following hospitalization for ACS revealed a lack of clear information about $\mathrm{CR}$ from HCP played a role in patient attendance with only $61 \%$ of patients comprehending some or all of the information provided to them in hospital (Hutchinson et al., 2015). Other barriers to participation include: the perception of CR as not beneficial for improving their overall health, inconvenient program times, being too standardized to meet individual needs (Clark et al., 2013) and lack of information on how to contact CR programs (Hutchinson, 2015). CR programs have also been viewed as lacking in social support and encouragement to attend from CR personnel for women specifically, and not being targeted towards the needs of women nor being sensitive to cultural norms (Clark et al., 2013).

\section{Contextual Factors Influencing Participation in Cardiac Rehabilitation}

\section{Education and Income}

Limited and varied information is available on the influence of education and income on CR attendance. Among the studies reporting on education level, individuals with a minimum of high school education were significantly more likely to attend CR (Leung et al., 2011; Parashar et al., 2012; Weingarten et al., 2011). In two studies CR participants were more likely to have attained post-secondary education (Dunlay et al., 2009; Sanderson et al., 2010). Mckee et al. (2014) also found higher education to be significantly associated with CR attendance, although

the specific level of education was not indicated. Two studies found no significant differences in education level between attendees and non-attendees at CR (Anderson \& Emery, 2014; Blair et al., 2014).

There is also evidence in the literature indicating that socioeconomic status can be a predictor of $\mathrm{CR}$ attendance. In a retrospective review of attendance and CR completion by level of neighbourhood income in Canada, Lemstra, Alsabbah, Rajakumar, Rogers and Blackburn 
(2013), found cardiac patients hospitalized for IHD, PCI or CABG, from lower-income neighbourhoods were significantly less likely to attend and complete CR. In a prospective study by Anderson and Emery (2014), findings revealed income to be a significant predictor of CR adherence, with lower reported income negatively associated with the number of CR sessions attended, although income parameters were not included in the study. Leung et al., (2011) found non participants reporting a lower family income $(<\$ 50,000)$ were significantly less likely to attend CR. Additional findings from Sanderson et al., (2010) reported higher referral rates as being associated with income reported as greater than $\$ 15,000$. Among ethnic minorities specifically in the cardiac population in one study, lower reported socioeconomic status was found to be an independent predictor of non-attendance at CR (Zhang, Sobolev, Prince \& Taub, 2014).

\section{Relationship Status and Support Systems}

Few studies were identified reporting on the relationship between CR attendance and relationship/marital status or friend/family support systems. Among the articles identified in this review, only six examined relationship status or social support as variables. Two studies found unmarried patients were significantly less likely to attend CR (Parashar et al., 2012; Weingarten et al., 2011). Although the findings were not significant, Blair et al., (2014) found CR attendees reported higher levels of social support systems compared with non-attendees. Findings from a recent systematic review also support the results of Blair et al., which found that lack of family

or friend support was a common barrier to CR attendance (Clark et al, 2013). Other studies found relationship status (Dunlay et al., 2009; McKee et al., 2014) or perceived social support (Parashar et al., 2012) did not influence CR participation. 


\section{Referral, Wait Times, and HCP Related Factors}

Lack of referral of eligible patients has been cited as a barrier to uptake of CR programs, especially among women, older populations, ethnic minorities, and those with lower socioeconomic status (Brown et al., 2009; Grace et al., 2013; Mampuya, 2012). In addition, lack of physician endorsement has been cited as a primary barrier for CR enrollment of HF patients (Grace et al., 2009). Uncertainty about the safety of exercise programs for HF, particularly in older persons, is a contributing factor which has resulted in a fewer eligible attendees being referred to CR (Grace et al., 2009). More recently, Grace et al., (2014) reported on physicianrelated reasons for lack of referrals to $\mathrm{CR}$ in Canada which include: lack of knowledge related to CR locations, physicians' perceptions of low patient motivation, and inconvenience of filling out and sending referral forms. In-hospital referral has been found to be a significant predictor of attendance at CR in Canada (Grace et al., 2011). There is also research suggesting different approaches to CR referral could have a positive impact upon CR enrollment. Grace et al., (2011) found enrollment rates between $44 \%$ and $73 \%$ using different referral strategies which included: automatic referral upon discharge from hospital (45\%), HCP endorsement and discussion about CR with the patient (44\%), a combination of automatic referral and HCP discussion (60\%) and other strategies including the provision of motivational letters and education materials about $\mathrm{CR}$ (73\%). Similar findings from Dunlay et al., (2009) revealed increased CR enrollment referred to CR in-hospital has also been associated with increased CR participation.

Wait times are another factor related to $\mathrm{CR}$ attendance. In a study examining the association between $\mathrm{CR}$ wait time and enrollment rates among various cardiac conditions, including HF, Russell et al., (2011) found longer wait times had a negative effect on CR enrollment. They reported that for each day of waiting, participants were $1 \%$ less likely to enroll. 


\section{Travel Factors}

CR travel time for individuals has been found to have a significant impact upon referral and program enrollment (Brual et al., 2010; Endo, Goto, Suzuki, Matusda, \& Yasumura, 2015). Brual et al., found distances of 60 minutes or greater to travel to CR was negatively associated with referral and patient enrollment, but not with degree of participation in CR once enrolled. Two studies exploring travel factors were inclusive of patients with an admitting diagnosis of HF (Endo et al., 2015; Turk-Adawi et al., 2014). In their study exploring factors associated with outpatient $\mathrm{CR}$ enrollment and adherence in patients with a primary diagnosis of HF, IHD, or post CABG, Endo et al., also found distance lived from CR was a significant predictor of enrollment with attendees reporting shorter driving distances to CR (mean distance of $10.7 \mathrm{~km}$ ) as compared with non-attendees (mean distance of $19.8 \mathrm{~km}$ ). In their systematic review, Murray et al., (2012) found both longer commute times and issues with transportation were independent predictors of non-uptake of CR programs. Conversely, Turk-Adawi et al., found individuals living in a rural location were significantly more likely to enroll in CR. Both Blair et al. (2014) and Dunlay et al., (2009) found no differences in the distance lived from CR programs from attendees versus non attendees.

\section{Other Factors}

Additional modifiable, patient-related barriers influencing CR attendance in Canada were assessed in two studies using the Cardiac Rehabilitation Barriers Scale (de Melo Ghisi, Oh, Benetti, \& Grace, 2013; Shanmugasegaram, Oh, Reid, McCumber, \& Grace, 2013). Predominant reasons cited for non-attendance were patients reporting already existent exercise routines at home or in the community, personal travel factors including distance travelled, time constraints, work responsibilities and other health issues, which are consistent with findings from Jackson et 
al., (2012) and Grace et al., (2009). Mean barrier scores were significantly and negatively

associated with CR adherence among CR participants attending a site-based program

(Shanmugasegaram et al., 2013). Other factors related to lack of CR participation reported by non-attendees include: the belief that $\mathrm{CR}$ would not be beneficial for improving health status or recovery and had confidence in their ability to recover without CR, dislike of groups, and lack of motivational support (Jackson et al., 2012).

\section{Gaps in the Literature}

The reviewed literature highlights the socio-demographic and clinical characteristics of individuals in studies who elected to attend or not-attend a CR program after referral. Several limitations were identified in this review. The primary limitation is the paucity of literature available specific to the HF cohort when exploring various cardiac populations and the sociodemographic, clinical, and contextual factors influencing CR participation. The primary diagnoses included in the articles reviewed included: $\mathrm{CAD}$, post-MI and post-CABG. Minimal studies included participants with HF and among these studies the majority only included HF as co-morbidity, while the primary study populations of interest were among other cardiac populations. Only five studies included HF as a primary diagnosis, however these studies were not representative of the Canadian context. No study was found in this review exploring factors associated with CR attendance solely in a HF patient population.

Due to the varying needs of different cardiac populations, much of the current literature may not be transferable to the HF population specifically. In addition, since exercise has only been recognized as safe and beneficial for patients with HF since 2009, there is limited research on this cohort specifically as compared with other cardiac populations where exercise has been recognized as a beneficial and integral component to heart health. Among studies that identified 
long wait times as a factor associated with non-attendance at CR, they did not explore what occurred during the time between referral and intake in $\mathrm{CR}$ that could have impacted an individual's decision to attend such as disease-specific complications, re-hospitalization, or other factors.

Among the studies included, the majority were retrospective reviews, which by nature may have incomplete information related to socio-demographic data of interest. Among the retrospective study designs reviewed, there was little data was available pertaining to income, education level, ethnicity, and relationship status or social supports limited in the literature reviewed. Few studies were conducted in Canada as the majority of studies reported in this review took place in the US with the inclusion of some international studies from Australia, England, Scotland, Ireland, Sweden, Poland, Belgium, Japan, and Israel. As health care systems and contexts may differ between countries, further research in a Canadian context is arguably necessary. As the majority of studies in this review took place in the United States where patients rely on insurance plans for coverage of CR or pay-out-of-pocket for these programs, the absence of universal health care coverage is a major factor differentiating the health care system, limiting generalizability to the Canadian context. Further prospective research is needed focusing on the socio-demographic and clinical factors associated with $\mathrm{CR}$ attendance versus non-attendance in the HF population in the Canadian health care setting.

\section{Summary}

Based on the current literature, $\mathrm{CR}$ is a safe and effective secondary prevention intervention in the HF population. There are a multitude of both individual and contextual influences associated with CR attendance among various cardiac populations including HF, CAD, MI, CABG, PCI, angiogram, and angina. The most frequently occurring diagnostic 
categories reported in the literature on $\mathrm{CR}$ attendance to date are post-MI, CAD and post-CABG. In this review barriers and facilitators to $\mathrm{CR}$ attendance were presented along with patient characteristics of attendees versus non-attendees. Key variables positively associated with CR attendance among cardiac populations included: younger age ( $<75$ years), male, Caucasian, higher income, higher level of education, living closer to a CR facility, and fewer co-morbidities. The main barriers identified included: lack of referrals by HCPs, distance to travel, lack of transportation, lack of support systems, negative views of CR and misconceptions of the benefits of attendance and illness perceptions.

Furthermore, the lack of representation of the HF population in the CR literature is a concerning finding that needs to be addressed. The limited literature available on HF specifically makes it difficult to draw conclusions related to the factors influencing enrollment, participation and adherence to $\mathrm{CR}$ in this population after referral. Further research reflecting $\mathrm{CR}$ in the Canadian health care system may reveal factors that differ from international studies. Results from this study may address some of the gaps identified in the literature to date which include: being specific to the HF patient population, a prospective study design, being conducted within a Canadian context, and including following up with patients to explore clinical complications experienced by patients with HF in the time period between referral and expected intake time to CR which no study has explored to date. 


\section{Chapter 3: Conceptual Framework}

The conceptual framework guiding this study emerges out of the Andersen and Newman (1973) Model of Health Service Utilization. This chapter provides an overview of the model as well as conceptual and operational definitions for the study key variables of interest. These include socio-demographic and clinical characteristics of HF patients related to those who intend to attend and those who do not intend to attend CR. The socio-demographic and clinical characteristics that were collected in this study include: age, gender, ethnicity, education level, employment status, income level, marital status, family supports, living arrangement, distance lived from CR facility, transportation access, New York Heart Association classification (I-IV), and co-morbidities.

\section{Andersen and Newman's Model of Health Care Service Utilization}

Andersen and Newman's (1973) Model of Health Service Utilization serves as a conceptual framework aimed at predicting and explaining use of health services, such as CR (Figure 1.0). The model was designed to focus on the individual person as the unit of analysis as relating to health service utilization (Andersen \& Newman, 1973). The multilevel model has been cited extensively in empirical literature as the guiding framework for studies (Babitsch, Gohl, \& von Lengerke, 2012). It has been applied to in-hospital, outpatient services, community centers, primary care, mental health, and physician services (Babitsch et al., 2012), as well as the CR setting to explore physician and patient-related factors that contribute to enrollment (Grace et al., 2010; Grace, Evindar, Kung, Scholey, \& Stewart, 2004). The model allows for conceptualization of the variation in utilization rates of health services, such as $\mathrm{CR}$, and because of this, was chosen as the guiding framework for this study. Although the model was developed in the 1970s, Andersen and Newman's model is considered seminal work, which continues to be 
used in the current literature to guide studies, as outlined above, and was chosen as it best fits with the study purpose.

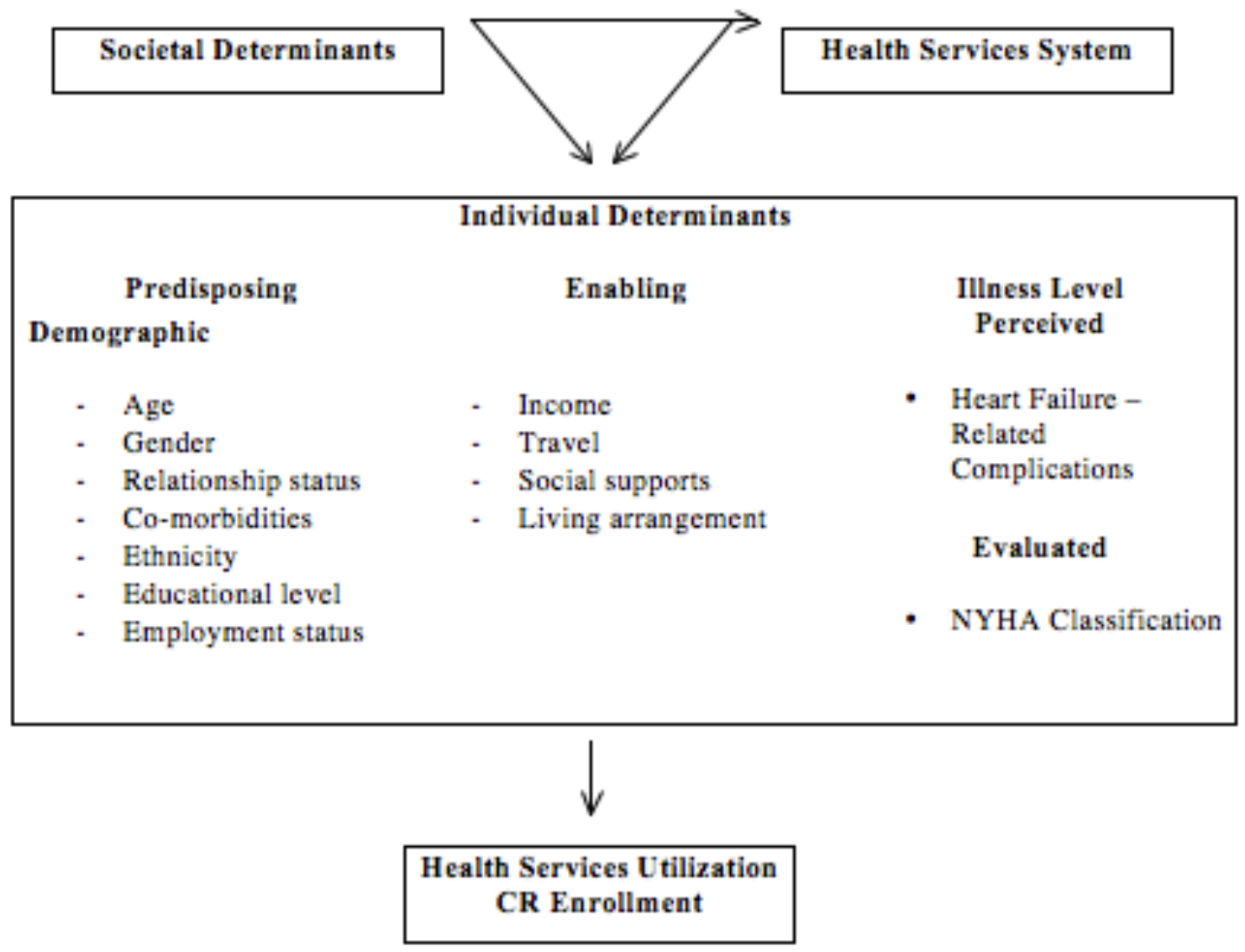

Figure 1.0 Health Care Utilization Framework For Analysis: Factors Associated With CR Enrollment in the Heart Failure Patient Population After Referral. This model was adapted for the purposes of this study from Andersen, R., \& Newman, J.F., 1973, The Milbank Memorial Fund Quarterly Health \& Society, 51(1), p. 14. Copyright 1973 by the Milbank Memorial Fund.

\section{Factors Influencing Health Service Utilization}

According to Andersen and Newman (1973), the use of health services is viewed as an individual behavior. The model is rooted in the behavioural sciences, which explain individual behavior as being a function of individual characteristics, the individual and their interaction with society, and the environment (Andersen \& Newman, 1973). In Andersen and Newman's model, the factors that influence health service utilization can be differentiated as societal 
determinants, those related to the health services system, and individual determinants. Societal determinants are the factors within society that bring about change in health service use such as new technology or policy changes (Andersen \& Newman, 1973). The health services system factors refer to the labour and financial resources allocated to health services and organization of health care delivery (Andersen \& Newman, 1973). Individual determinants are described as being a function of three factors: predisposing characteristics, factors that enable individuals to use the health system and illness level, as perceived by the individual themselves or evaluated by a HCP. This study focuses on the individual determinants of health service utilization as related to CR participation.

\section{Individual Determinants of Health Service Utilization}

\section{Predisposing Factors}

Predisposing factors refer to the socio-demographic and cultural characteristics of an individual that may increase the likelihood of an individual's use of health services, which include demographics, social structure and health beliefs (Andersen \& Newman, 1973). Demographic characteristics included in Andersen and Newman's model are age, gender, marital status and past illnesses, or co-morbidities. Social structure refers to the status of an individual in their community and is typically influenced by education, occupation, and ethnicity (Andersen \& Newman, 1973). Finally, health beliefs are the attitudes held by an individual towards the use of health services (Andersen \& Newman, 1973).

\section{Enabling Factors}

Anderson and Newman (1973) recognize that although individuals may be predisposed to health service use because of individual factors, enablers need to be in place to provide them with a means to do so effectively. Enabling factors that can influence health service use include: 
higher level of income, caregiving or support networks, shorter travel times and wait times for health services.

\section{Illness Level}

Illness level is the third factor of the individual determinants of health care utilization (Andersen \& Newman, 1973). Illness level involves both perceived and evaluated level of illness by the individual (perceived) and by HCPs (evaluated) (Andersen \& Newman, 1973). Individuals' perception of their illness level can have an impact upon their beliefs about their needs for health services. Measures of perceived illness in the model can include an individual's self-evaluated level of illness as well as condition-specific symptoms experienced in a given time period, such as HF-related complications. In addition, the objective evaluation by HCPs, once individuals access the health care system, can also have an impact upon subsequent health service use based on the HCPs' recommendation or referrals for additional care. Measures of evaluated illness include: physical assessments by clinicians as well as clinical judgment related to illness severity, e.g. NYHA classification.

\section{Application of Andersen and Newman's Model of Health Care Service Utilization to CR Participation}

Anderson and Newman's (1973) model provides a framework for understanding patterns and trends in individual health service utilization. The framework combines demographic characteristics with social and environmental factors to predict health service use, which ultimately can influence health outcomes. As the purpose of this study was to explore sociodemographic characteristics related to HF patient intent to attend or not attend CR after referral, the Andersen and Newman model was chosen as the guiding framework. Specifically, the model provided the basis for the investigator-generated socio-demographic variables of interest in this 
study. This study focused specifically on Andersen and Newman's individual determinants of health care utilization, exploring predisposing factors, enabling factors and illness level as per the model.

The variables of interest that were assessed in this study according to Andersen and Newman's (1973) model included: age, sex, relationship status, co-morbidities, ethnicity, education level, employment status, income, transportation access, family supports, living arrangement, distance lived from CR facility, transportation access, New York Heart Association Classification (I-V), and HF-related complications The conceptual and operational definitions of the study key variables are discussed below.

\section{Predisposing Factor Variables}

Age.

Age was conceptually defined as the number of years that have passed since an individual's date of birth. Operationally, age was measured from participant self-report and verified in the participant's legal medical record.

\section{Gender.}

Gender was conceptually defined as the "socially constructed roles, behaviours, expressions and identities of girls, women, boys, men, and gender diverse people. It influences how people perceive themselves and each other, how they act and interact, and the distribution of power and resources in society," (Canadian Institute of Health Research, 2015). Operationally, gender was collected based on participant self-report. 


\section{Relationship status.}

For this study, relationship status was conceptually defined as an individual's involvement with a significant other. Operationally, relationship status was collected based on participant self-report.

\section{Co-morbidities.}

Multiple definitions have been provided in the literature for co-morbidities, which are conceptually defined as the presence of one or more co-occurring clinical conditions (Valderas, Starfield, Sibbald, Salisbury, \& Roland, 2009). According to Valderas et al., the definition of comorbidity is "most often defined in relation to a specific index condition" (p.358), with the index condition varying depending on the research questions of the study. For this study, the specific index condition was a diagnosis of heart failure and all subsequent conditions were identified as co-morbidities. Operationally, co-morbidities were identified from a review of the participant's self-report and verified in participant's legal medical record.

\section{Ethnicity.}

For this study, ethnicity was conceptually defined as a group of individuals that identify with one another based on shared ancestry, language, and culture. Operationally, ethnicity was collected based on participant self-report.

\section{Education level.}

For this study, education level was conceptually defined as the highest level of education the individual has completed. Operationally, education was collected based on participant selfreport. 


\section{Employment status.}

Employment status was conceptually defined by the Statistics Canada (2010) labour force status classification of an individual as employed (working at a job or business that is paid, or unpaid work contributing directly to a family business such as a farm, or employed while off on disability, illness, personal and family factors), unemployed (the unutilized supply of labour, without a job, laid off, or seeking employment), or not in the labour force. Operationally, employment status was collected based on participant self-report.

\section{Enabling Factor Variables}

\section{Income.}

For this study, income was conceptually defined as the annual amount of money received by an individual through work or by other means. Operationally, income was collected based on participant self-report.

\section{Travel.}

For this study, travel was conceptually defined as the individuals ability to access transportation to attend CR, the mode of transportation used and the distance the individual has to travel from his or her place of residence to attend CR. Operationally, travel was collected by participant self-report of access to and mode of transportation.

\section{Social supports.}

For this study social support was defined as having family or friends that a participant identified as being accessible and reliant in a time of need. Operationally, social supports were measured by the participant self-report of having individuals, such as friends, family, caregivers, or neighbours to rely on in times of need. 


\section{Living arrangements.}

For this study living arrangements were defined as the current individual(s) with whom the participant shares a place of residence. Operationally, living arrangement was collected from participant self-report.

\section{Illness Level Variables}

\section{New York Heart Association Classification (NYHA) (I-IV).}

NYHA classification is defined by the American Heart Association (AHA) (2016) as the classification system for HF to classify the severity of HF from I-IV based on functional limitations. Class I HF refers to no limitation during physical activity (AHA, 2016). Class II HF refers to slight limitations during regular physical activity, resulting in fatigue, SOB, or palpitations (AHA, 2016). Class III HF refers to significant limitations during any physical activity resulting in fatigue, SOB, or palpitations (AHA, 2016). Class IV refers to the inability to carry on any physical activity without severe symptoms of HF, and having symptoms of HF at rest (AHA, 2016). Operationally, the NYHA classification was measured based on the classification made by the participant's HCP, collected through review of the patient's legal medical record.

\section{HF-related complications.}

For this study, HF complications were conceptually defined as the physical symptoms experienced by participants indicating the heart is not pumping adequate amounts of blood to meet the needs of the body, or worsening heart failure. Operationally, HF-related complications were measured at weeks one, three, five and six after discharge from hospital using an investigator-generated checklist of HF-complications (Appendix A). The identified complications were based on the most frequently cited HF clinical symptoms and complications 
evaluated in the literature (Health Quality Ontario, 2015; Jurgens et al., 2009; Lee \& Moser, 2012; Albert, Trocheman, Li, \& Lin, 2009).

\section{Research Questions}

The following research questions guided the study. The questions were based on the premise that all patients have been referred to $\mathrm{CR}$.

1) What are the socio-demographic and clinical characteristics of patients with HF who intend to attend CR?

2) What are the socio-demographic and clinical characteristics of patients with HF who do not intend to attend CR?

3) What are the number and type of complications reported in patients with HF who intend to attend CR?

4) What the number and type of complications reported in patients with HF who do not intend to attend CR?

5) What are the barriers to participation for patients who do not intend to attend CR? 


\section{Chapter 4: Methods}

The methodology used in conducting this descriptive pilot study is outlined in this chapter. The following components are detailed: study design, setting, sampling, ethics, inclusion and exclusion criteria, data collection procedures, and data analysis procedures.

In this study, all patients received a referral to $\mathrm{CR}$ at the time of hospital discharge as part of standard discharge practice for the HF patient population at the hospital where the study was conducted.

\section{Study Design}

A descriptive pilot study design was used to address the research questions and examine the feasibility of conducting a larger scale study to describe the socio-demographic and clinical characteristics as well as HF-related complications associated with HF participant's intention to attend or not attend CR after being referred. A descriptive design is most appropriate for exploring and describing a phenomenon of interest and related variables in a naturalistic setting, which is congruent with the purpose of this study that was conducted in a naturally occurring setting (Grove et al., 2013). The purpose of a pilot study is to examine the feasibility of the study design, measures, participant recruitment, data collection methods and analysis, and adequacy of instrumentation and is useful for the generation of new knowledge where limited research has been previously conducted on a phenomenon of interest (Grove et al., 2013; Moore, Carter, Nietert, \& Stewart, 2012). Presently, no study was found in the current literature examining factors influencing HF patient attendance at referred for CR. Thus, pilot data from this study may be used to inform the successful design and execution of a larger scale study. 


\section{Setting}

Participants were recruited from two inpatient cardiology units at two large, urban academic teaching hospitals within the same organization in Ontario, Canada. The majority of patients admitted to these units are commonly diagnosed with heart failure, coronary artery disease, arrhythmias, cardiomyopathy, and congenital heart conditions.

\section{Sampling}

The inpatient cardiology units were chosen as the setting for recruitment as this allowed for timely and convenient access to a large number of potential participants in the same location. Individuals were recruited for the study through a non-probability, convenience-sampling method.

Participant screening for eligibility to participate in this study was established by the researcher in consultation with unit Patient Care Coordinators, who were provided with a copy of the inclusion and exclusion criteria for this study. The primary nurse was informed by the researcher about patients who were eligible to participate and provided with a script to use to inform potential participants of the study (See script in Appendix B). Specifically, the primary nurse informed eligible patients that a study was being conducted and asked patients if they would like to hear more about the study. If a patient expressed interest in learning more about the study or participating, the primary nurse obtained verbal consent from the patient for the researcher to approach the patient. The researcher then approached the patient in person to further explain the study and begin the process of obtaining informed consent utilizing a script (Appendix C). The researcher provided patients with a letter outlining the study purpose (Appendix D), and asked if they had any questions, prior to obtaining informed consent in person. 
This study aimed for a sample size of 30 participants. A power analysis is not applicable for a pilot study, as the primary objective is to determine feasibility and acceptability of design procedures, including sampling methods, to be used for the possible development of a larger scale study (Leon, Davis \& Kramer, 2011; Grove et el., 2013). The data generated from a pilot study is used for determining sample size if there are no other studies available to calculate effect size for the intervention(s) (Grove et al., 2013). The sample size in a pilot study is based on pragmatics, or what is realistic, for recruitment (Leon, Davis \& Kraemer, 2011). The number of participants for this study was chosen based on realistic sampling methods for this particular study and the literature, which recommends between 10 - 30 participants (or greater) for preliminary pilot studies (Browne, 1995; Grove et al., 2013). The possibility of participant attrition (i.e., the number of participants who dropout of the study) may well have an impact upon this study (Grove et al., 2013). The study estimated a 5\% - 10\% dropout rate, which was accounted for in the pilot study by aiming for a sample size of 30 participants (Grove et al., 2013). Therefore, if a 5-10\% dropout rate occurs, the sample size would still be adequate according to the recommendations for sample sizes in pilot studies of $10-30$ participants (Grove et al., 2013).

\section{Ethics}

Ethics approval for the study was obtained from both Ryerson University and the hospital Research Ethics Board. Ethical conduct in this study was upheld in accordance with the three core principles in the Tri-Council Policy on Ethical Conduct for Research Involvement Humans: respect for persons, concern for welfare, and justice (Canadian Institute of Health Research (CIHR), Natural Sciences and Engineering Research Council of Canada (NSERC), \& Social Sciences and Humanities Research Council of Canada (SSHRC), 2014). 
Respect for persons involves a moral obligation of the researcher to respect participant autonomy, which includes obtaining informed consent and ensuring ongoing consent (CIHR, NSERC, SSHRC, 2014). Concern for welfare aims to protect the welfare, or physical, mental, spiritual wellbeing, and physical, economical and social circumstances, of participants (CIHR, NSERC, SSHRC, 2014). This includes maintaining privacy and confidentiality of participant information, minimizing potential risks of harm for participation, and providing participants with information about foreseeable risks and potential benefits of study participation (CIHR, NSERC, SSHRC, 2014). Justice refers to the fair and equitable treatment of persons (CIHR, NSERC, SSHRC, 2014). The recruitment process and inclusion criteria, which are justified in this study, are important components of upholding justice in research conduct. The voluntary nature of participation was ensured by having the study introduced by a member of the potential participant's circle of care (i.e.: the primary nurse) but ensuring that the consent process was carried out by the researcher, who was outside the circle of care.

Informed consent involves ensuring the participants understand the research purpose, study design and procedures, foreseeable risks and benefits to the participants (CIHR, NSERC, SSHRC, 2014). Participants were provided with the opportunity to engage in an informed consent process and given detailed written and verbal information about the study including study purpose, data collection procedures, and duration of participation in the study. Participants were given an opportunity to ask questions, informed of the voluntary nature of participation as well as their right to withdraw from the study at any time without negative consequences (See the study consent form included as Appendix E). Participants were informed by the researcher their decision to attend or not attend CR did not affect their care in-hospital or follow-up care in any way and that their health care practitioners in their circle of care would not be made aware of 
their decision to attend or not. In addition, potential participants were also offered the opportunity to take the consent form home to review further and discuss with a trusted confidante before providing informed consent to attend.

Participants were informed that the results of the study may help to generate knowledge for health care practitioners on the characteristics of patients who intend to attend CR, identify gaps in transitional care planning from hospital to $\mathrm{CR}$, and improve upon referral processes for CR. Participants were also informed there were no direct benefits to participation in the study or foreseeable risks of physical harm, however they may potentially feel uncomfortable, anxious or distressed discussing their diagnosis or current state of health during follow up telephone calls with the researcher.

A designated research phone and phone number was utilized by the researcher for contacting participants during the course of the study at the outlined data collection periods. Participants were informed they could contact the research investigator at any time throughout the study via telephone call or university e-mail, which is only accessible by the researcher.

Participant confidentiality was maintained by assigning a code number to participant names for all data collected during the study. A master list of participants and contact information with the study codes were kept separately from the consent forms and all other study data in a secure area at the hospital. All hard copy data was stored in a secure office, in a locked drawer at the hospital, which only the researcher had access to. Electronic data were stored on a secure, password protected hospital server. Only de-identified participant data were transferred from the hospital to Ryerson University on a secure encrypted flash drive for data analysis by the researcher and thesis supervisor. Study data will be kept in a secure storage area in the hospital for a period of 10 years as per recommendations by the hospital Research Ethics Board. 


\section{Inclusion and Exclusion Criteria}

The target population for this study was any individual living with a diagnosis of HF, New York Heart Association (NYHA) classification I-IV, presenting with or without comorbidities and being referred to $\mathrm{CR}$ at the time of hospital discharge. The primary physician or nurse practitioner automatically refers all stable patients with HF to CR as part of the standard discharge care on the unit. The referral forms are directly sent from the hospital to the CR center closest to the patient's place of residence and once they are reviewed and processed (which will vary depending on the CR facility), the patient will receive a telephone call from the rehab facility to schedule an initial visit. The initial visit may include a physical assessment and education or exercise components depending on the CR facility.

Inclusion criteria for the study were:

Eligible participants were adults, 18 years of age or older, with a new or existing diagnosis of HF with the ability to provide consent for self, without identified cognitive impairments. Participants were discharged from hospital with a referral to $\mathrm{CR}$, aligning with the purpose of the study. Since referrals to CR occur at the time of discharge from hospital and are sent to CR centers directly from hospital, recruitment on the inpatient unit allowed for the most accessible recruitment location to obtain consent and information to follow-up with participants at subsequent data collection periods. Participants were required to comprehend and communicate in English, as all data collection methods and communication was undertaken in English. There was no mechanism for translation services for this study due to the small sample size and lack of funding. Participants were required to have access to a telephone as the data collection procedures involve telephone calls from the researcher to the participant. 
Exclusion criteria were:

Any patients with a diagnosis of HF who were not referred to CR were not eligible for participation in this study. Patients with severe or life-threatening co-morbidities were also excluded from the study, as this may have possibly prevented inclusion in the study due to terminal disease or inability to exercise at CR. Patients were excluded if they were awaiting heart transplant and had or were waiting for left ventricular assistive devices, as these individuals have unique treatment needs and their condition may impact eligibility or ability to attend or complete CR.

\section{Data Collection Procedures}

Data were collected through the use of a demographic survey (See Appendix F for demographic data collection sheet) administered at the enrollment visit, after the participant had provided consent. The content for the demographic survey was based on socio-demographic data collected in the CR literature and identified as factors influencing CR attendance. The thesis committee, four content experts, reviewed the content in the demographic survey for face validity. Participants were contacted by telephone at 4 time points after the enrollment visit, at weeks 1, 3, 5 and 6 to obtain information on HF-related complications post-discharge from hospital (See Appendix A).

During the enrollment visit, participants were asked to provide socio-demographic information to the researcher, who read the questions from the questionnaire to the participant and recorded their response on the demographic questionnaire. The demographic information collected included: age, ethnicity, gender, relationship status, education level, employment status, income level, travel factors (distance lived from CR facility, transportation access), family/friend supports, living arrangement, NYHA classification (I-IV), and co-morbidities. 
Age, ethnicity, and gender were measured based on the individuals self-report and confirmed in participants' legal medical record. Original categories were collapsed and re-coded so the data could be analyzed quantitatively. The following variables were re-coded: ethnicity, relationship status, employment status, level of income, living arrangement, level of education, transportation method, and travel time. The original codes are presented, along with the recoding of the variables of interest in this study. Based on participant responses in two categories, gender was then coded as male (1), female (2). Relationship status was coded as being single (1), married (2), divorced (3), separated (4), widowed (5), common-law (6), and other (7). Relationship status was re-coded as single (1) (inclusive of divorced, separated and widowed) and in a relationship (2) (inclusive of codes married, common-law, and other if participants indicating they were a relationship). Education level was coded as none (1), elementary (2), high school (3), college (4), undergraduate (5), professional degree (6), graduate degree (7). Education level was re-coded as high school or less (1) (inclusive of none, elementary and high school), college/undergraduate (2), and professional/graduate (3). Employment status was coded as employed (1), unemployed (2), retired (3), and volunteer (4). Employment status was re-coded as working (1) (inclusive of code 1) and not working (2) (inclusive of unemployed, retired, and volunteer). Personal income level was coded as less than $\$ 30,000$ (1), $\$ 30,001-50,000$ (2), $\$ 50,001-70,000$ (3), $\$ 70,001$ or greater (4), prefer not to say (5). Income level was re-coded as 50,000 or less (1) and 50,001 or greater (2) and prefers not to say (3). Travel was examined based on individual's having access to transportation to CR which was coded as yes (1) and no (2) with no recoding necessary for this variable. The distance lived from CR, which was coded as 30 minutes or less (1), 45 minutes (2), 1 hour (3), and over 1 hour (4). Distance lived from CR was re-coded as 30 minutes or less (1) and Greater than 30 minutes (2). Type of transportation 
used to access CR was coded as driving self (1), family/friend driving (2), volunteer driver (3), taxi (4), public transit (5), other (6). Type of transportation was re-coded as driving self (1) and reliance on transportation (2) (inclusive of family/friend driving, volunteer driver, taxi, public transit, and other if it included reliance on other types of transportation). Family/friend supports, or having individuals to rely on in times of need was coded as yes (1) and no (2). Living arrangements were coded as living alone (1), with a spouse/common-law (2), with family/friends (3), and other (4). Living arrangements was re-coded as living alone (1) and living with other(s) (2) (inclusive of spouse/common-law, family/friends, and other if the participant stated they lived with another individual).

The researcher collected information on NYHA and co-morbidities from the patient discharge summary in the participant's medical chart completed by the patient's primary physician or nurse practitioner. NYHA classification was coded based on levels I-II (1) and IIIIV (2). No recoding was necessary for this variable.

The socio-demographic and clinical information collected were determined based on the review of the literature, which revealed factors influencing CR participation among various cardiac populations (see Chapter 2). Participants were also asked to provide telephone contact information for the researcher to contact them during the follow-up data collection period (See Patient Contact Form in Appendix G).

The researcher contacted participants via telephone at one week following hospital discharge, and at week 3 , week 5 , and week 6 for a total of 4 follow-up telephone calls postdischarge to collect data on common HF-complications based on the presence or absence of symptoms. HF-related complications adapted from the HF Signs and Symptoms checklist (Albert, Trochelman, Li, \& Lin, 2010) (See HF-related Complications Survey included as 
Appendix A). Albert et al., reported content validity of the HF Signs and Symptoms checklist was demonstrated by an expert HF panel which included three advanced practice nurses and a cardiac patient educator. Internal consistency was not reported.

The researcher utilized a script during the follow-up telephone conversation with participants (See Telephone Call script included as Appendix H). The researcher read aloud the HF- related complications one at a time to the participant. Participants were asked to answer "yes" or "no" to the questions outlined in Appendix H.

During the final data collection telephone call (week 6) participants were asked about HF-related complications and if they had attended or intended to attend the CR program. For the purpose of this study, attendance at CR refers to attending the first day of the rehabilitation program, where activities include exercise and/or educational components, and not the initial intake appointment that takes where baseline assessments are completed to ensure participants are stable prior to starting the program. Participants were asked to answer "yes" or "no" if they had attended the CR program. If participants were not yet enrolled in the CR program at the end of six weeks, participants were asked to answer "yes" or "no" if they were planning to attend CR.

\section{Data Analysis Procedures}

Data were analyzed using descriptive statistics, Fishers exact test, or independent samples t-test. The level of significance for this study was set at $\mathrm{p}<0.05$. Data were analyzed using SPSS (Version 22) software.

Continuous variables were reported as a mean and standard deviation and frequency and percentages were reported for categorical variables. Fishers exact test was used to determine if there were significant differences in nominal demographic data (gender, ethnicity, education 
level, employment status, marital status, social supports, living arrangement, transportation access, NYHA classification) among participants who either intended or did not intend to attend CR. This test was chosen as an alternative to using Chi-Square test of independence due to cell counts having a frequency below five, which violates the assumptions required run Chi-Square analysis in which all cells should have expected frequencies greater than or equal to five. Fishers exact test has the following assumptions: variables are measured at the nominal level, categories are mutually exclusive, that is participation in one category does not allow participation in another category.

The independent samples t-test was used to determine differences in age and number of co-morbidities among participants intending to attend and not intending to attend CR.

Information about barriers to participation in CR among participants who indicated they did not intend to attend CR were explored by asking participants reasons or potential barriers to nonparticipation and frequency of responses were reported. 


\section{Chapter 5: Results}

In this chapter, findings related to demographic and clinical characteristics of intentions to attend $\mathrm{CR}$ in the HF population are presented. HF-related complications experienced up to 6 weeks after hospital discharge and barriers to CR attendance are presented. Results of the data analysis are discussed in sections of this chapter relative to the study research questions.

\section{Participation}

A total of 42 patients were eligible and approached by the patient's primary nurse to participate in this study. Of the 42 patients who were asked about their interest in the study, 34 agreed to meet with the researcher and were consented to participate in the study. The researcher did not explore reasons for non-participation due to the nature of recruitment, with the study initially being presented to patients by their primary nurse. If patients declined to hear more about the study, the researcher did not make contact with the patient. Among the 34 patients who were enrolled in the study, four participants did not complete the study due to withdrawal $(n=1)$ and being lost to follow-up ( $n=3)$. Data from a total of 30 participants who were enrolled and completed this study were used in the final statistical analysis (Figure 2.0).

Figure 2.0: Flow of Participants Through the Study

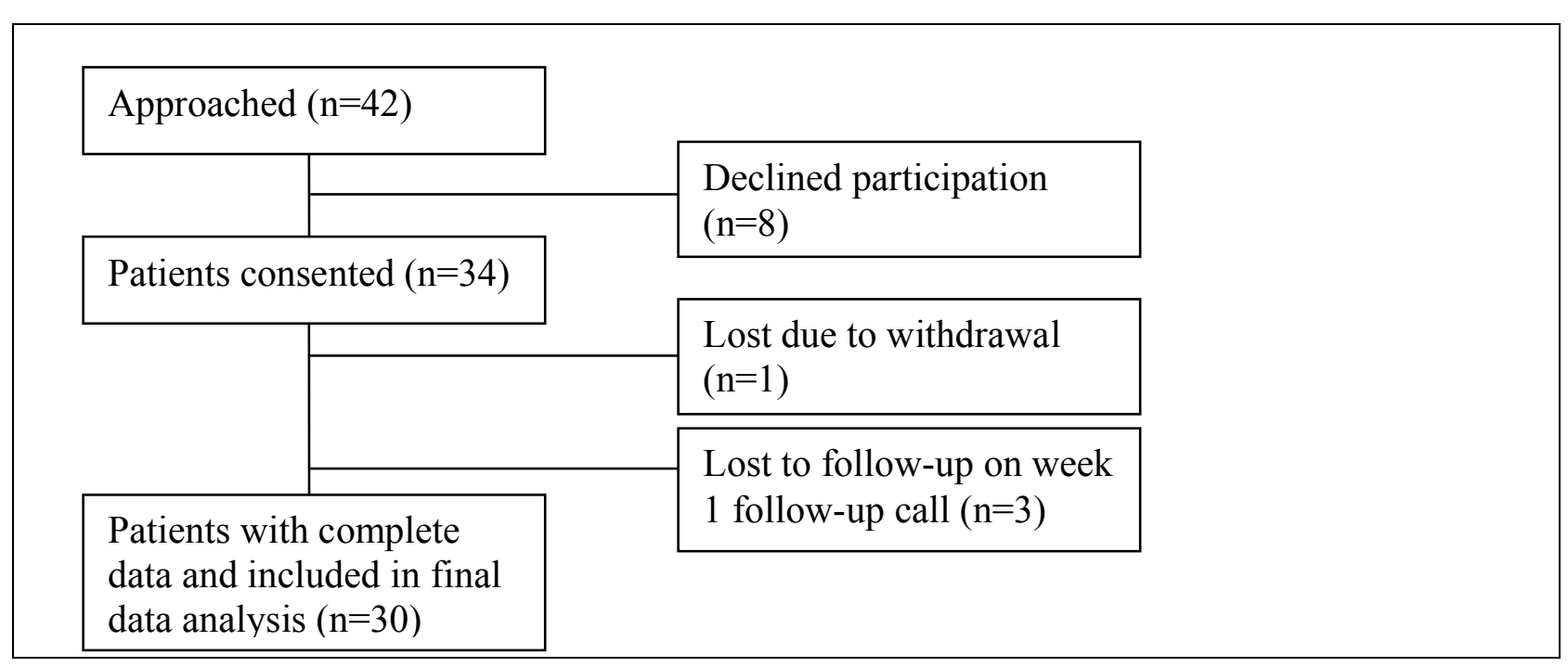


The age of participants in this study ranged from 45 - 86 years, among which $21(70 \%)$ were male and $9(30 \%)$ were female. Participants were predominantly Caucasian $(76.7 \%)$, followed by Asian (10\%), African American (6.7\%), Middle Eastern (3.3\%) and Hispanic (3.3\%). Among the 30 participants in this study, 11 (36.7\%) intended to attend CR (9 males, 2 females) and 19(63.3\%) did not intend to attend CR (12 males, 7 females).

Research Questions 1 and 2: What are the socio-demographic and clinical characteristics of patients with HF who intend to attend CR? What are the socio-demographic and clinical characteristics of patients with HF who do not intend to attend CR?

\section{Demographic Characteristics}

Demographic characteristics of the sample are outlined in Table 1. Significant differences between participants who intended to attend CR and did not intend to attend CR in age, employment status, income level, and transportation method were noted (Table 2). Participants who intended to attend $\mathrm{CR}(\mathrm{n}=11)$ were significantly older $(\mathrm{M}=78.8$ years, $\mathrm{SD}=7.3$, age range of 67-86 years) than those who did not intend to attend $(\mathrm{M}=66.3$ years, $\mathrm{SD}=10.9$, age range $51-80$ years; $\mathrm{t}(28)=3.38, \mathrm{p}=.002$, two-tailed) in CR. Findings suggest individuals who intended on participating in CR were significantly more likely to be unemployed/retired $(90.9 \% \mathrm{vs} .52 .6 \%$, $\mathrm{p}=.049)$ and have an income level $>50,000(81.8 \%$ vs. $21.1 \%, \mathrm{p}=.002)$. Furthermore, participants intending to attend CR were also significantly more likely to be able to drive themselves to CR, without relying on transportation from family/friends or public transit system (81.8\% vs. $21.1 \%, p=.002)$ than those not intending to attend CR.

As shown in Table 2, there were no significant differences found between participants who intended to attend CR and those who did not intend to attend CR in relation to gender $(p=0.419)$, ethnicity $(p=1.00)$, relationship status $(p=0.466)$, living arrangement $(p=0.140)$, 
education level $(p=0.132)$, travel time $(p=1.00)$ and support system $(p=0.611)$. The majority of participants intending to attend $\mathrm{CR}$ or not intending to attend $\mathrm{CR}$ were predominantly male (81.8\% and $62.2 \%$ respectively), and Caucasian ( $72.7 \%$ and $78.9 \%$ respectively). Although, in both CR attendance intended and CR not intended groups, the majority of participants reported being single (72.7\% and 57.9\% respectively) more participants who did not intend to attend CR reported being a relationship (married or common-law) (42.1\%) compared with those intending to attend (23.7\%). As well, the majority of participants intending to attend CR reported living with others (spouse/common-law/family or friends) $(81.8 \%)$ compared to those not intending to attend CR (52.6\%). Although there were no significant findings in relation to education level, the majority of individuals intending to attend $\mathrm{CR}$ reported education at the college/university level (63.6\%) compared with those not intending to attend (31.6\%). Participants intending and not intending to attend CR reported a similar travel time of 30 minutes or less to $\mathrm{CR}$ ( $54.5 \%$ and $47.4 \%$ respectively) and identified having a support system in times of need $(81.8 \%$ and $89.5 \%$ respectively). 
Table 1. Baseline characteristics

\begin{tabular}{|c|c|c|c|c|}
\hline \multirow[t]{2}{*}{ Profile } & \multicolumn{2}{|c|}{$\begin{array}{l}\text { CR Attendance Intended } \\
(\mathrm{n}=11)\end{array}$} & \multicolumn{2}{|c|}{$\begin{array}{l}\text { CR Attendance Not Intended } \\
\qquad(\mathrm{n}=19)\end{array}$} \\
\hline & $\mathrm{n}$ & Mean $( \pm$ SD)(range) & $\mathrm{n}$ & Mean $( \pm \mathrm{SD})($ range) \\
\hline \multirow[t]{2}{*}{ Age } & 11 & $78.8( \pm 7.3)(67-86)$ & 19 & $66.3( \pm 10.9)(51-80)$ \\
\hline & $\mathrm{n}$ & $\%$ of sample & $\mathrm{n}$ & $\%$ of sample \\
\hline \multicolumn{5}{|l|}{ Gender } \\
\hline Male & 9 & 81.8 & 12 & 63.2 \\
\hline Female & 2 & 18.2 & 7 & 36.8 \\
\hline \multicolumn{5}{|l|}{ Ethnicity } \\
\hline Caucasian & 8 & 72.7 & 15 & 78.9 \\
\hline Asian & 2 & 18.2 & 1 & 5.3 \\
\hline African American & - & - & 2 & 10.5 \\
\hline Middle Eastern & - & - & 1 & 5.3 \\
\hline Hispanic & 1 & 9.1 & - & - \\
\hline \multicolumn{5}{|l|}{ Relationship Status } \\
\hline Single & 3 & 27.3 & 3 & 15.8 \\
\hline Married & 3 & 27.3 & 8 & 42.1 \\
\hline Divorced & 2 & 18.2 & 5 & 26.3 \\
\hline Separated & - & - & - & - \\
\hline Widowed & 3 & 27.3 & 3 & 15.8 \\
\hline Common-law & - & - & - & - \\
\hline Other & - & - & - & - \\
\hline \multicolumn{5}{|l|}{ Employment Status } \\
\hline Employed & 1 & 9.1 & 9 & 47.4 \\
\hline Unemployed & 1 & 9.1 & 1 & 5.3 \\
\hline Retired & 9 & 81.8 & 9 & 47.4 \\
\hline Volunteer & - & - & - & - \\
\hline \multicolumn{5}{|l|}{ Level of Income } \\
\hline$<30,000$ & - & - & - & - \\
\hline $30,001-50,000$ & 2 & 18.2 & 15 & 78.9 \\
\hline $50,001-70,000$ & 8 & 72.8 & 4 & 21.1 \\
\hline$>70,001$ & 1 & 9.1 & - & - \\
\hline Prefer not to say & - & - & - & - \\
\hline
\end{tabular}




\begin{tabular}{|c|c|c|c|c|}
\hline \multicolumn{5}{|l|}{ Living Arrangement } \\
\hline Alone & 2 & 18.2 & 9 & 47.4 \\
\hline Spouse/Common-law & 5 & 45.5 & 8 & 42.1 \\
\hline Family/Friends & 4 & 36.4 & 2 & 10.5 \\
\hline Other & - & - & - & - \\
\hline \multicolumn{5}{|l|}{ Level of Education } \\
\hline None & - & - & - & - \\
\hline Elementary & 2 & 18.2 & 2 & 10.5 \\
\hline High school & 2 & 18.2 & 11 & 57.9 \\
\hline College & 5 & 45.5 & 4 & 21.1 \\
\hline Undergraduate & 2 & 18.2 & 2 & 10.5 \\
\hline Graduate Degree & - & - & - & - \\
\hline Professional Degree & - & - & - & - \\
\hline \multicolumn{5}{|l|}{ Support System } \\
\hline Yes & 9 & 81.8 & 17 & 89.5 \\
\hline No & 2 & 18.2 & 2 & 10.5 \\
\hline \multicolumn{5}{|l|}{ Transportation Access } \\
\hline Yes & 11 & 100 & 19 & 100 \\
\hline No & - & - & - & - \\
\hline \multicolumn{5}{|l|}{ Transportation } \\
\hline \multicolumn{5}{|l|}{ Method } \\
\hline Driving self & 9 & 81.8 & 4 & 21.1 \\
\hline Family/friend & 2 & 18.2 & 7 & 36.8 \\
\hline Volunteer & - & & - & - \\
\hline Taxi & - & & 2 & 10.5 \\
\hline Public Transit & - & & 6 & 31.6 \\
\hline Other & - & & - & - \\
\hline \multicolumn{5}{|l|}{ Travel Time to $\mathrm{CR}$} \\
\hline 30 minutes or less & 6 & 54.5 & 9 & 47.4 \\
\hline 45 minutes & 3 & 27.3 & 2 & 10.5 \\
\hline 1 hour & 1 & 9.1 & 6 & 31.6 \\
\hline Over 1 hour & 1 & 9.1 & 2 & 10.5 \\
\hline
\end{tabular}


Table 2. Baseline characteristics (categories collapsed for analysis)

$\begin{array}{ccc}\text { CR Attendance } & \text { CR Attendance Not } & \$ p-V a l u e \\ \text { Intended } & \text { Intended } \\ (\mathrm{N}=11) & (\mathrm{N}=19)\end{array}$

\begin{tabular}{lccc}
\hline \hline n & $\begin{array}{c}\text { Mean } \\
( \pm \text { SD })(\text { range })\end{array}$ & n & $\begin{array}{c}\text { Mean } \\
( \pm \text { SD })(\text { range })\end{array}$ \\
\hline
\end{tabular}

Age $11 \quad \begin{array}{cccc}78.8( \pm 7.3) & 19 & 66.3( \pm 10.9) & (51-80)\end{array} \quad+.002$

\begin{tabular}{lccccc}
\hline & n & \% of sample & n & \% of sample & \\
\hline Gender & & & & & \\
Male & 9 & 81.8 & 12 & 63.2 & .419 \\
Female & 2 & 18.2 & 7 & 36.8 &
\end{tabular}

\begin{tabular}{lccccc}
\hline Ethnicity & & & & \\
Caucasian & 8 & 72.7 & 15 & 78.9 & 1.00 \\
Other Ethnic Groups & 3 & 27.3 & 4 & 21.1 &
\end{tabular}

\section{Relationship Status}

Single

In a relationship

$8 \quad 72.7$

$3 \quad 23.7$

11

8

57.9

42.1

.466

\section{Employment Status}

Working

Not Working

$\begin{array}{cc}1 & 9.1 \\ 10 & 90.9\end{array}$

9

10

47.4

52.6

$\uparrow .049$

\section{Level of Income}

$<50,000$

$>50,001$

$\begin{array}{ll}2 & 18.2 \\ 9 & 81.8\end{array}$

15

4

78.9

21.1

$\uparrow .002$

\section{Living Arrangement}

Alone

Not Alone

2

2
9

18.2

81.8

9

10

47.4

52.6

.140

\section{Level of Education}

None/Public/High school

College/University

Graduate/Professional

$\begin{array}{cccc}4 & 36.4 & 13 & 68.4 \\ 7 & 63.6 & 6 & 31.6 \\ - & - & - & -\end{array}$

68.4

31.6

.132

Transportation Method

Driving self

Reliance on transportation

$\begin{array}{ll}9 & 81.8 \\ 2 & 18.2\end{array}$

4

15

21.1

78.9

$\uparrow .002$ 
Travel Time to $\mathbf{C R}$

$\leq 30$ minutes

$\begin{array}{llll}6 & 54.5 & 9 & 47.4\end{array}$

$>30$ minutes

5

45.5

10

52.6

1.00

\section{Support System}

Yes

No

9

2

81.8

17

89.5

18.2

2

10.5

$\$ \mathrm{p}$ is significant at $\leq 0.05$.

$\uparrow$ significant difference between CR intended and CR not intended groups

\section{CR-Related Factors}

Participants who intended to attend CR were significantly more likely to have had a discussion with their HCP about CR participation prior to discharge from hospital compared with those that did not intend to attend ( $90.9 \%$ vs. $26.3 \%$ respectively, $\mathrm{p}=0.002)$. Results six weeks post-hospital discharge revealed no participants had yet begun a CR program (i.e., none had attended the exercise and/or educational components of the program). Less than half of participants in both the intention to attend $\mathrm{CR}$ and no intention to attend $\mathrm{CR}$ groups had received a phone call from $\mathrm{CR}$ that provided them with a scheduled start date for the program $(45.5 \%$ and $36.5 \%$ respectively).

\section{Clinical Characteristics}

Participants intending to attend CR had significantly $(\mathrm{p}=.009)$ lower NYHA classification of $\mathrm{HF}$, with $72.7 \%$ of these individuals having a functional classification of I-II compared with only $21.1 \%$ of participants who did not intend to attend CR.

Participants who did not intend to attend CR had a higher average number of co-morbid conditions $(\mathrm{M}=5.1, \mathrm{SD}=2.2)$ than those who intended to attend $(\mathrm{M}=3.5, \mathrm{SD}=1.9, \mathrm{t}(28)=1.98$, $\mathrm{p}=.058$, two-tailed) in CR, although these results were not significant. 
Table 3. Clinical characteristics and CR related factors (discussion with HCP, intention to attend $C R$, start date for $C R$ ) between participants who intended to attend $C R$ and those who did not intend to attend $C R$.

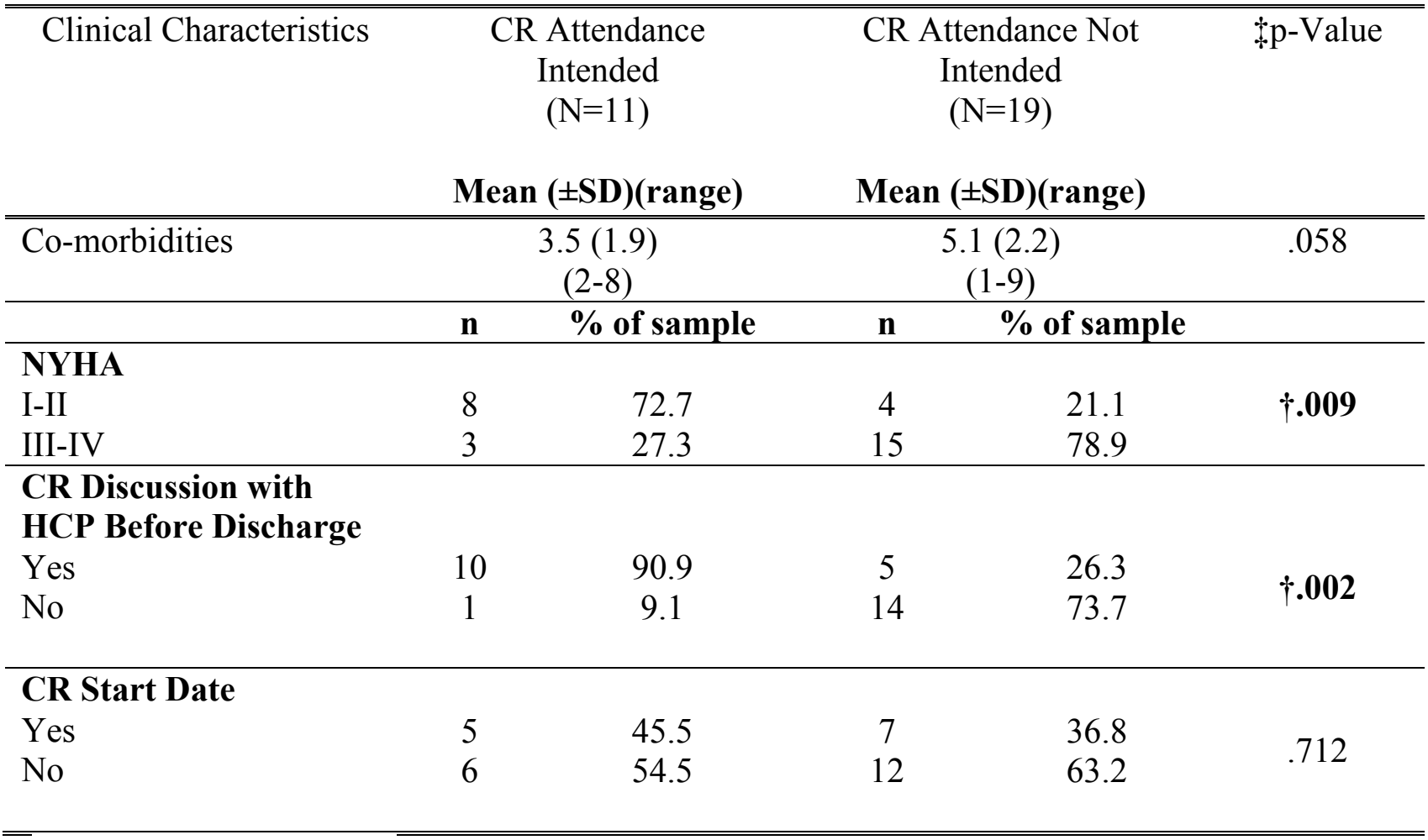

$\$ \mathrm{p}$ is significant at $\leq 0.05$

$\dagger$ significant difference between CR intended and CR not intended groups

Research Questions 3 and 4: What are the number and type of complications reported in patients with HF who intend to attend CR? What the number and type of complications reported in patients with HF who do not intend to attend CR?

To address the research questions, participants were asked to recall their experiences of HF-related complications at follow-up periods of weeks one, three, five and six after discharge from hospital. Results are presented as frequencies based on participants who answered, "yes" to having experienced a HF complication over the six-week follow-up period. Results for the total number of reported experiences of each HF complication are presented in Figure 3. HF complications reported each week are displayed as frequencies in Figures 4, 5, and 6. 


\section{Total HF Complications}

Over the course of six weeks, those intending to attend CR reported experiencing a total of $35 \mathrm{HF}$ complications compared with $80 \mathrm{HF}$ complications experienced by those not intending to attend. Range of symptoms reported per patient was 0-10 among individuals intending and not intending to attend CR. Individuals not intending to attend reported a higher number of total HFrelated complications experienced each week than those who intended to attend CR during each follow-up call: week one (30 vs. 4 respectively), week three (29 vs. 16 respectively), week five (15 vs. 11 respectively), and week six (6 vs. 4 respectively). The most frequently experienced complications for the entire follow-up period were the same for both those intending to attend $\mathrm{CR}$ and not intending to attend CR those which were fatigue ( 8 vs. 24 respectively), followed by sleeping difficulty (6 vs. 12 respectively), and SOB (6 vs. 10 respectively). After fatigue, sleeping difficulty and SOB, those intending to attend CR experienced orthopnea (5), edema (2), dizziness (2) and exercise intolerance (2) as the next highest reported complications. Those not intending to attend CR experienced exercise intolerance (8), weight loss (6) and lack of appetite (6). Symptoms of palpitations, coughing, or changes in urine output were not reported in either group during the study period. 
Figure 3: Total HF Complications

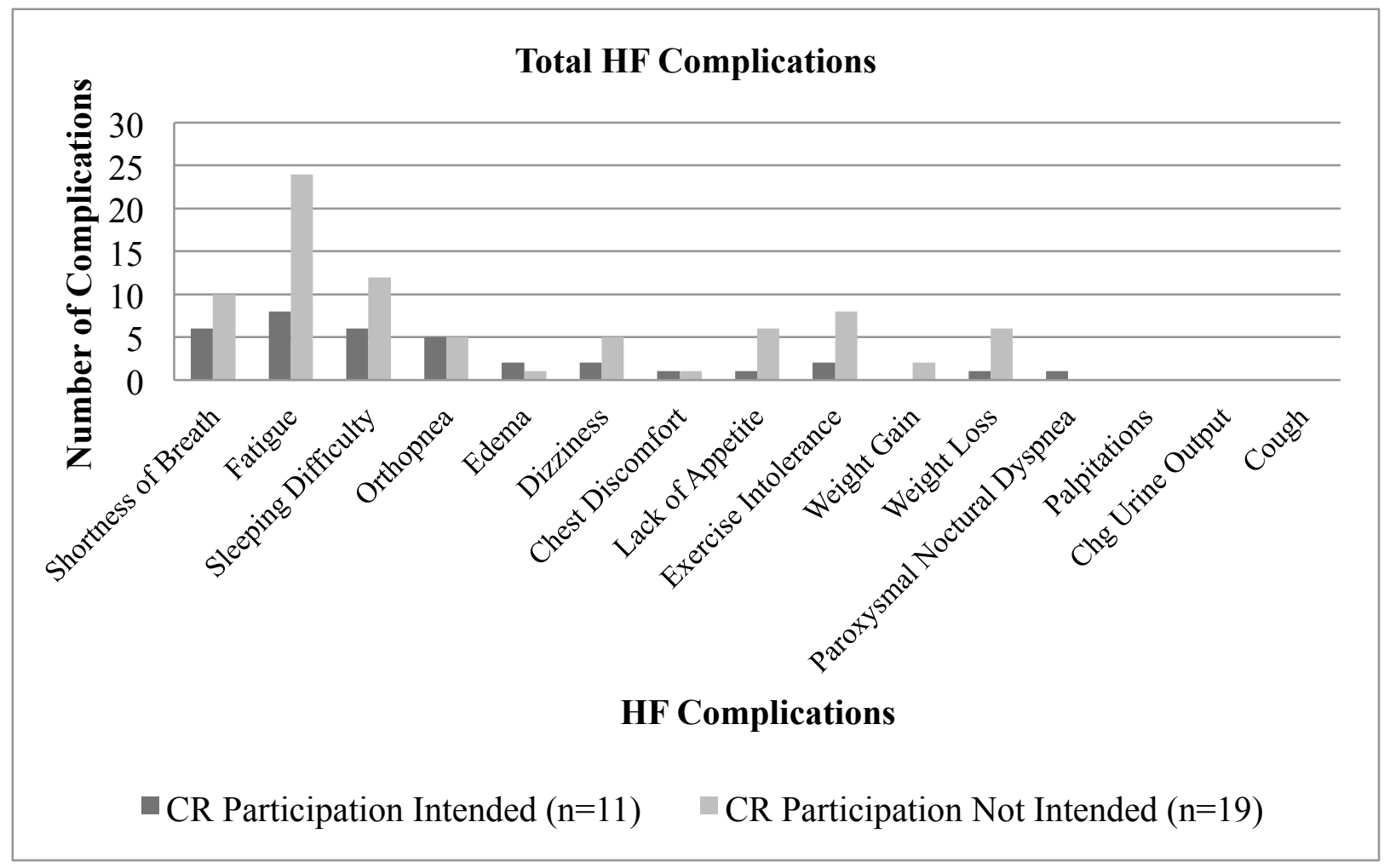

\section{HF Complications: Weekly and Bi-Weekly Follow-up}

Within the follow-up period of this study at weeks one, three, five and six, participants who did not intend to attend CR experienced the highest number of complications during weeks one (30) and three (29), while participants who intended to attend CR had the highest complications in weeks three (16) and five (11). While total complication scores were similar between the two groups at weeks five and six, the most notable differences in reported complications occurred in the first week post-discharge with the CR non-participation group reporting a total of 30 symptoms compared with the CR participation group reporting only 4 complications. 
Figure 4: HF Complications Week One

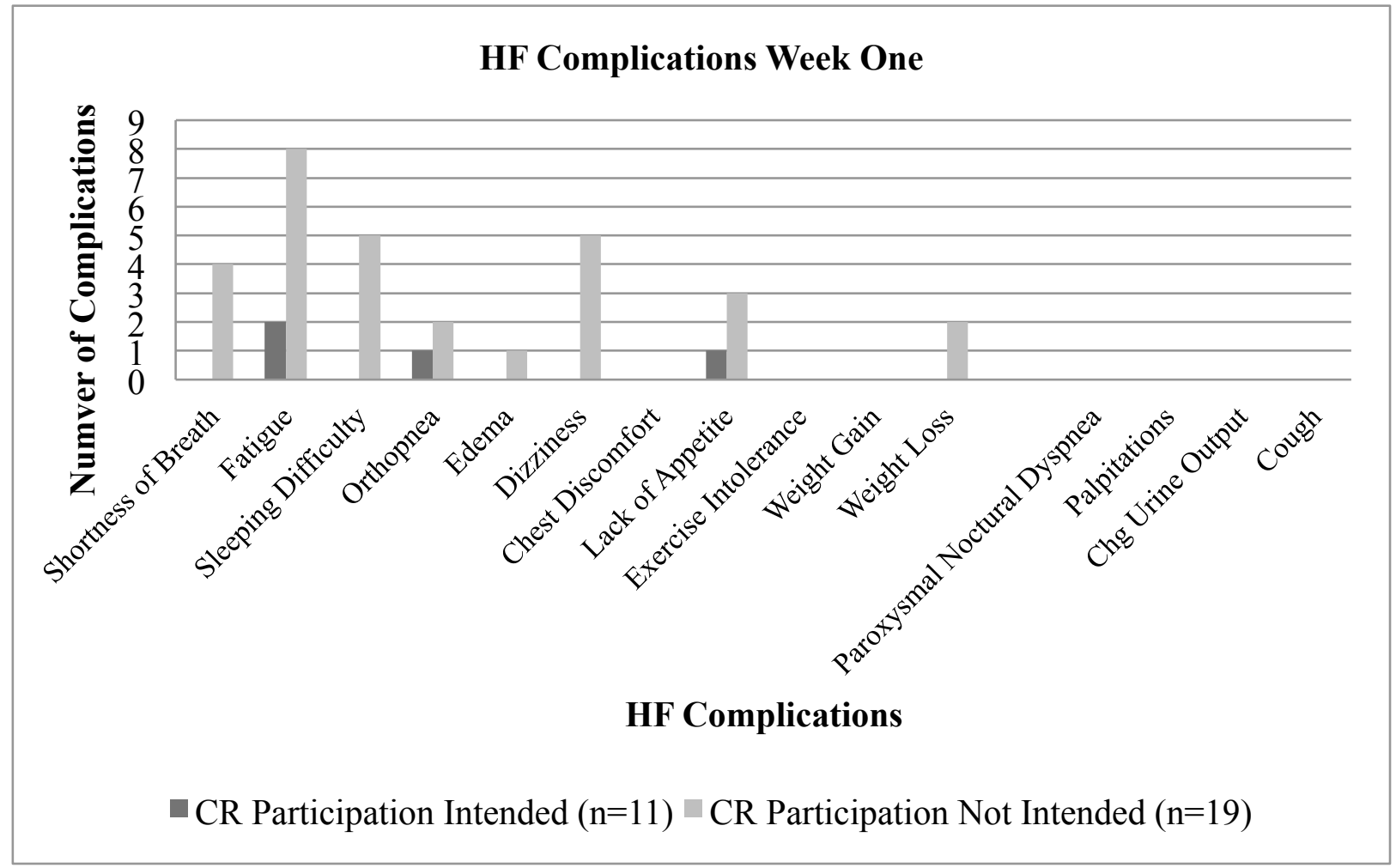

Figure 5: HF Complications Week Three

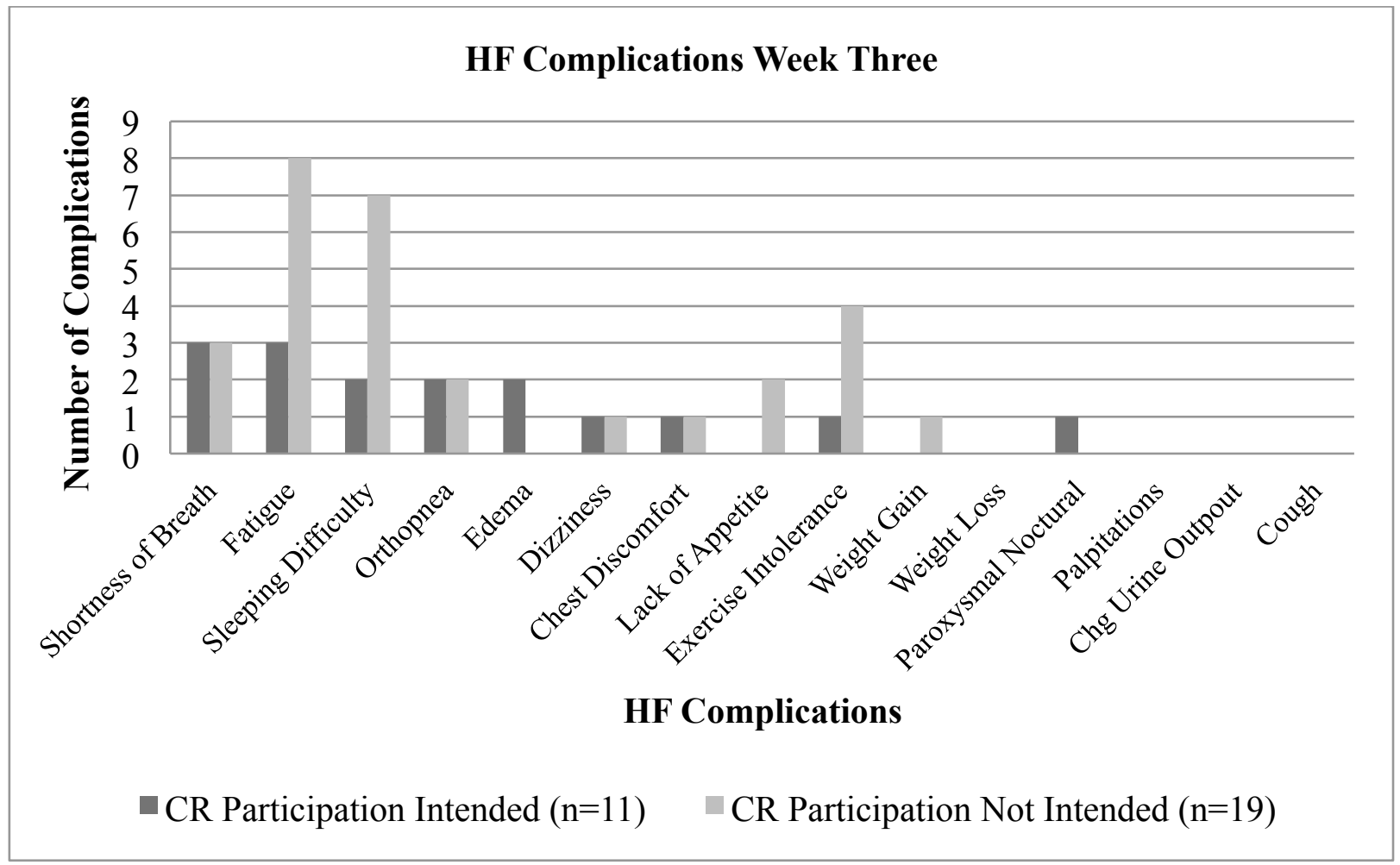


Figure 6: HF Complications Week Five

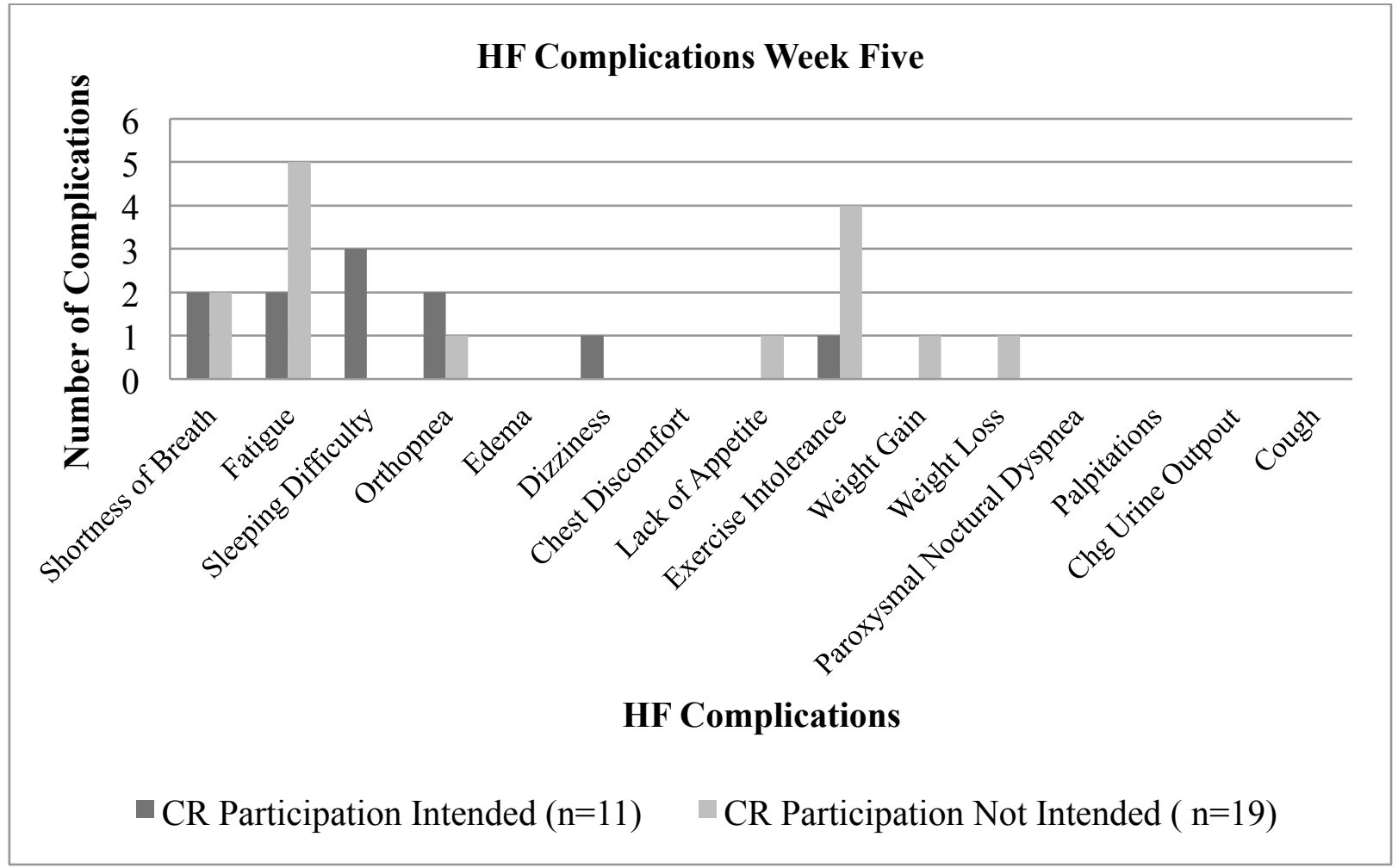

Figure 7: HF Complications Week Six

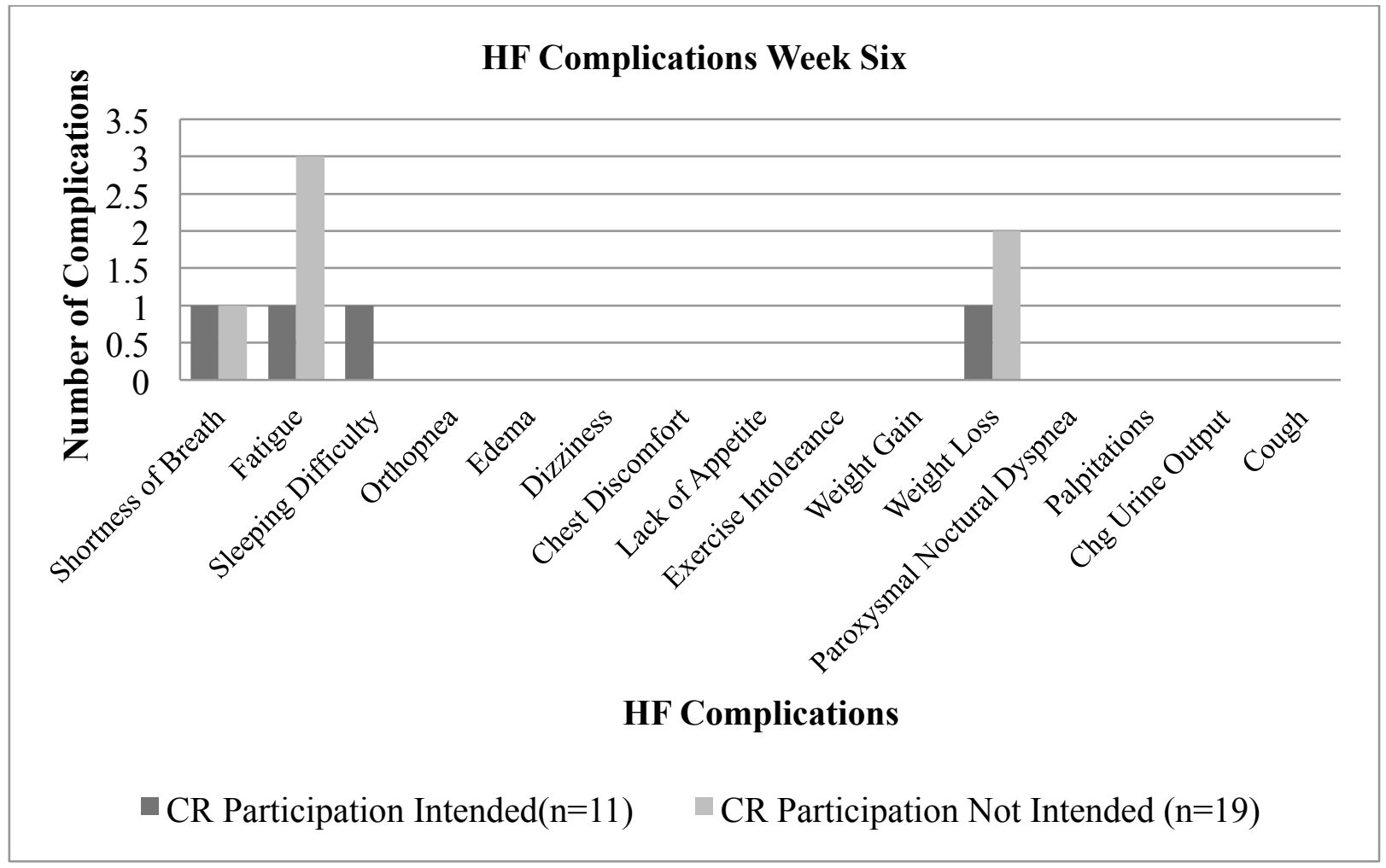


Research Questions 5: What are the barriers to participation for patients who do not intend to attend CR?

\section{Barriers to CR}

The 19 participants who did not intend to attend CR provided information about barriers to attendance, with some participants reporting multiple barriers (Table 4). The most frequent cited barrier by over half (52.6\%) of participants was related to lack of information provided to them about the CR program including why they were referred and the potential health benefits of the program, followed by lack of time to attend due to full-time employment commitments (36.8\%). Participants (21.1\%) also reported they were unable to attend as they relied on family members for transportation, of whom were reported as unable to provide transportation during the day when the program was offered. Other barriers to participation included travel time related to the location of the CR program being too far from home (15.8\%), not feeling physically well enough to attend (15.8\%), and having no interest in participating $(10.5 \%)$.

Table 4. Barriers to CR Participation by Participants Not Intending to Attend CR (n=19)

\begin{tabular}{lcc}
\hline \hline Barriers to CR Participation & Frequency & $\%$ \\
\hline $\begin{array}{l}\text { Lack of information about CR } \\
\text { and benefits to attendance }\end{array}$ & 10 & 52.6 \\
\hline $\begin{array}{l}\text { Employment } \\
\text { Family unable to drive }\end{array}$ & 7 & 36.8 \\
\hline $\begin{array}{l}\text { Travel time to CR } \\
\text { Not feeling well enough to }\end{array}$ & 3 & 21.1 \\
\hline \begin{tabular}{l} 
No intend \\
\hline \hline
\end{tabular} & 2 & 15.8 \\
\hline
\end{tabular}




\section{Chapter 6: Discussion and Implications}

This chapter includes a discussion of the research findings as they relate to the current literature and Andersen and Newman's (1973) conceptual model of Health Service Utilization. Implications for Nursing practice, policy, and education are also presented along with areas for further research.

This quantitative descriptive study was a pilot study to test the feasibility of examining socio-demographic and clinical characteristics of patients with HF who intend or do not intend to attend CR programs after referral, prior to designing a future large-scale study. While multiple studies have examined the role of CR in improving health outcomes, they have all primarily focused on cardiac diagnoses other than HF. No study found to date has specifically explored factors associated with CR attendance in the HF population. The present study explored differences in the demographic and clinical profiles and HF-related complications up to six weeks after hospital discharge of patients intending and not intending to attend CR. Barriers to CR attendance among those who had indicated they did not intend to attend CR were also explored. Identification of characteristics associated with intentions not to attend CR is required in order to explore potential strategies to optimize CR participation by eligible patients and improve clinical outcomes in this patient population.

\section{Key Findings}

Andersen and Newman's (1973) model aligned with the purpose of this study, providing a conceptual framework to further understand which factors may impact participation at CR. This thesis specifically addressed the individual determinants impacting health care utilization which includes three factors: predisposing characteristics, factors that enable individuals to use the health system and illness level, as perceived by the individual themselves or evaluated by 
HCPs. The results of this study are reflective of the three factors that make up the individual determinants of health service utilization, such as $\mathrm{CR}$, and a general discussion of the findings in relation to each of these factors are discussed below.

\section{Predisposing Characteristics}

According to Andersen and Newman (1973), predisposing factors include sociodemographic characteristics of individuals existing prior to their illness and diagnosis as well as health beliefs. This study found a number of differences in predisposing factors (sociodemographic and clinical characteristics), among participants intending to attend CR and those who did not intend to attend CR. Characteristics of the sample did not differ from the literature in terms of gender and ethnicity with females and various ethnicities being underrepresented in this study (Hutchinson et al., 2015; Leung et al., 2011; Piotrowicz et al., 2010). Among the females enrolled in this study $(n=9)$, the majority $(n=7)$ did not intend to attend $C R$, which is also consistent with findings in the literature that men are significantly more likely to enroll in CR (Doll et al., 2015; Martin et al., 2015; McKee et al., 2014).

The study findings of this pilot related to gender may be explained by findings from a recent meta-synthesis exploring qualitative studies of gender and access to CR (Angus et al., 2015). In this study, Angus et al. found the current delivery of CR programs may not meet the needs of potential participants. Specifically, traditional views of gender roles and responsibilities were found to be a main barrier to CR participation as women reported prioritizing homemaking and family caregiving responsibilities over CR (Angus et al., 2015). Gender differences in financial means to afford CR and lack of familial support compared to men also influenced CR attendance as women reported having to buy time to attend $\mathrm{CR}$, such as paying for child care or house cleaning services, and were found to have less familial encouragement to attend CR than 
men. Angus et al. reported that studies found women felt CR programs were similar to a male dominated gym and valued the social connections and emotional aspects of $\mathrm{CR}$, suggesting women's only programs would tailor to these needs. Angus et al., highlight the need for the use of theory in future studies to guide gender analysis and understand the social processes and other contextual factors that influence gender disparities in accessing $\mathrm{CR}$, such as socioeconomic status, age, format of CR programs (i.e.: group based or individualized, availability of programs) and cultural views which have also been implicated in influencing CR attendance. Thus, Angus et al., suggest future research focus less on the 'experiences of women', which may differ among individuals (i.e. not all woman have caregiving or household responsibilities and may be career focused) and more on the social determinants of access to CR.

Participants intending to attend CR were significantly older, and more likely to not be working. In this study, not working included both being unemployed and retired. In this study 20 participants were identified as not working (10 in the CR intended group and 10 in the CR not intended group). Among the 10 participants in each group, $90 \%$ were retired and $10 \%$ were unemployed. These findings are in contrast to the majority of literature reviewed for this study in which older age has been found to be a significant predictor of non-attendance, with patients over 65 least likely to access CR (Hutchinson, 2015; Leung et al., 2011; Martin et al., 2012; Turk-Adawi et al., 2014). However, the study findings are consistent with Mikkelsen, Korsgaard Thomsen, and Tchijevtich (2014) who examined factors related to non-attendance and dropout from $\mathrm{CR}$ and found younger age and employment commitments were significant predictors of non-attendance. A possible explanation for findings in this study is that the older participants were more likely to be retired or unemployed and therefore able to accommodate the times CR programs are typically offered, on weekdays during working hours. Lack of automatic referral 
processes in previous studies may have resulted in physician referral patterns less likely to refer older participants to CR (Grace et al., 2009; Hutchinson et al., 2015). Lack of time due to work commitments among younger participants was the most frequent reported barrier to CR attendance in this study. Multiple studies have also found work commitments to be a primary barrier to CR attendance and adherence (Mikkelsen et al., 2014; Shamnugasegaram et al., 2013).

Although the findings were not statistically significant, results of this study indicate relationship status, living arrangement, and education status are influencing factors of intention to attend $\mathrm{CR}$. Participants intending to attend $\mathrm{CR}$ were more likely to have education at the college/university level (63.6\% versus 31.6\%). This finding corresponds with other studies in which CR participants have a significantly higher level of education (high school or above) than non-participants (Dunlay et al., 2009; Sanderson et al., 2010). Study participants intending to attend CR were more likely to be single ( $72.7 \%$ vs. $57.9 \%)$ and living with others such as a partner, family or friends ( $81.8 \%$ vs. $52.6 \%)$. Few studies are available for comparison in the literature, with inconsistent findings as well as inconsistent categories of measurement for relationship status, with married/non married being the most frequently reported. Dunlay et al., did not find relationship status (married/not married) to be a significant predictor of CR participation, whereas two other studies found being married was a significant predictor of attendance (Parashar et al., 2012; Weingarten et al., 2011). These findings are important variables to consider and may suggest that the presence of social support at home have an influence upon CR participation more than relationship status.

\section{Enabling Factors}

Enabling factors include financial and organizational factors that serve as conditions that enable individuals to use health services. The enabling factors examined in this study were 
having a higher income, shorter travel times, having access to transportation, presence of a support system, and CR endorsement from HCPs prior to discharge. This study found patients with lower income $(<50,000)$ were significantly less likely to intend to attend CR. Similar findings from the literature suggest patients of lower socioeconomic status are less likely to attend CR (Leung et al., 2011) and also experience greater barriers to attendance, such as transportation issues, parking costs, and fewer health benefits to cover time off work for health prevention programs (Shanmugasegaram et al., 2013; Valenica, Savage \& Ades, 2011). Participants intending to attend CR also were significantly more likely to have access to a vehicle to drive to $\mathrm{CR}$, which has been a consistent finding in the literature, reported as a significant predictor of CR attendance (Dunlay et al., 2009; Murray et al., 2012). These findings suggest transportation factors are significant barriers to $\mathrm{CR}$ attendance for individuals who are unable to drive themselves or may not be able to afford transportation costs. Exploration of various models of CR delivery to address these modifiable barriers and increase uptake should be considered (Dalal et al., 2010).

Conflicting findings on the influence of travel time on CR attendance have been reported in the literature with both further distance having a significant negative impact on attendance (Shamnugasegaram et al., 2013) and no impact on CR attendance (Blair et al., 2014). Travel time to $\mathrm{CR}$ was not an influencing factor on $\mathrm{CR}$ attendance in this study, with similar travel times reported in both groups. Approximately half of participants in both groups in this study reported being within 30 minutes of $\mathrm{CR}$ (54.5\% intending to attend CR and $47.4 \%$ not intending to attend), with the majority of the remaining sample being within 30-60 minutes of CR. Only one participant in the CR intended group and two participants in the CR not intended group reported a travel time greater than 1 hour. These findings are consistent with a study that took place in 
Ontario by Brual et al., (2010) who found $88.8 \%$ of participants lived within a 60 minute drive time of a CR program as well as similar findings from another Canadian study that found $86 \%$ travelled 30 minutes or less to CR (Grace et al., 2015). Findings from this study and the literature suggest the majority of patients are within the acceptable threshold of 30 minutes to a CR facility as outlined by the Cardiac Rehab Network of Ontario (Suskin, MacDonald, Swabey, Arthur, Vimr...Tihaliani, 2003).

\section{Illness Level}

Factors that are associated with uptake of health services (e.g., CR) in this study included: patient perceptions of their level of illness through the evaluation of HF complications; patients' evaluated level of illness such as severity of HF based on NYHA classification scores; and number of co-morbidities reported by their HCPs. In this study the number of complications reported by participants in the follow-up time points (weeks one, three, five and six) ranged from 0-10 in both groups (CR attendance intended and not intended). Similarly, utilizing the HF Signs and Symptoms checklist, Albert et al., (2010) found the number of HF-symptoms reported ranged from 1 to 15 among patients in two groups: those who recalled symptoms one week prior to hospital admission and those who were followed up with in the ambulatory care setting one week post-discharge from hospital. The top reported symptoms in this study included fatigue, difficulty sleeping, SOB, orthopnea, and exercise intolerance. Similarly Albert et al., found the top five symptoms reported were fatigue, SOB, orthopnea, edema and dizziness, and decreased ability to exercise, with a higher number of symptoms reported by those with worsening NYHA functional class scores. Participants not intending to attend CR reported a higher number of symptoms experienced during all follow-up weeks of this study and were significantly more likely to have a NYHA functional class score of III-IV than those electing to attend. This finding 
indicates that an individual's intention to attend in CR can be negatively influenced by an increased number and severity of symptoms following hospital discharge. Identification of patients at risk for experiencing a higher number of HF-related complications (e.g. patients with NYHA scores of III-IV) prior to discharge should be considered so that appropriate support strategies for symptom identification and early intervention could be implemented. These support strategies could include referral to telehomecare programs and ensuring patients have a follow-up appointment with their family physician scheduled prior to hospital discharge. Earlier intake into CR programs may also serve to lessen symptoms as patients would have access to HCPs and monitored on a weekly basis.

Although not significant, findings from this study revealed participants with a higher number of co-morbidities were less likely to intend to attend CR, which is consistent with other research (Doll et al., 2015; Grace et al., 2009; Leung et al., 2011). Among reported barriers to attendance in this study, it is not surprising individuals electing not to attend CR reported feeling "too unwell to attend" based on the clinical findings in this study. Jackson et al., (2012) also found worsening clinical status in the presence of multiple health conditions to be a barrier to attendance, suggesting additional support and resources may be necessary to address symptoms of HF while participants await intake to CR or perhaps earlier intake into CR may mitigate symptoms.

\section{Rationale for Non-Significant Findings}

Factors identified as non-significant in influencing participant intention to attend CR in this study included: gender, ethnicity, relationship status, living arrangement, education level, travel time, support system, and co-morbidities. Possible reasons for findings related to gender and ethnicity may be due to the homogenous sample in this study, which lacked a representation 
of women or various ethnicities due to the small sample size. In addition, this may be due to the institution where recruitment took place, which is in an area where a large number of Caucasian/European individuals live in comparison to outside of the city core where more ethnicities are represented. Non-significant travel times found between the two groups may also be due to the study taking place in the downtown core of a densely populated major city where many individuals may live in close proximity to CR centres thereby reducing travel time, or rely on public transportation rather than driving which may increase travel time. Although some participants in both groups (intention to attend CR and no intention to attend CR) had identified receiving a CR start date, no participants had yet begun the program. This non-significant finding may be due to the increased referral rates to $\mathrm{CR}$ with the previous implementation of automatic referral processes that may result in longer processing times of referrals or may be due to program capacity issues. In addition, this study was inclusive of recruiting participants of all NYHA functional classification scores (I-IV). The severity of HF and symptoms experienced by individuals with a functional classification score of I (no limitation of physical activity) compared with functional classification scores of III or IV (marked limitations in physical activity and discomfort with an physical activity respectively) may have had an impact on individual's intention to participate irrespective of other co-morbidities, relationship status or living arrangements, education level or identified support systems which were all found to be non-significant. The small sample size in this study may also be a contributing factor to these non-significant findings. Future studies with a larger sample size may result in significant findings for these variables. 


\section{Implications}

\section{Implications for Practice}

The results of this study based on the examination of socio-demographic and clinical characteristics associated with intention to attend CR suggest HCPs should target selected HFpatients who are at risk for not attending (younger, employed, lower income and education levels, non married, and multiple co-morbidities, and NYHA III-IV functional classification). A very important consideration should be on the HF-related complications participants experienced in the early weeks following hospital discharge. The majority of participants in the study reported the highest number of complications in the first one to three weeks post-discharge which may have contributed to participants not feeling well enough to attend CR, a primary barrier in this study. Earlier intake into CR may facilitate earlier intervention for HF-related complications or reduce the number of complications reported among HF patients.

Results also suggest the importance of endorsing CR in the inpatient setting. A discussion with patients about their CR referral by one of their HCPs was a significant factor associated with participant intention to attend CR in this study. Lack of information about CR and the benefits of the program was the most frequently reported barrier to attendance in this study. In a recent systematic review by de Melo Ghisi, Polyzotis, Oh, Pakosh, and Grace (2013) HCP endorsement prior to discharge was significantly related to $\mathrm{CR}$ enrollment. Further patient education to include reasons for referral, components of CR, HCP endorsement, and additional education resources should be a standard of clinical practice and shared responsibility among interprofessional team members (Arena, Williams, Forman, Cahalin, Coke, Myers,...Lavie, 2012; de Melo Ghisi et al., 2013).

Findings suggest the need for HCPs to explore potential strategies to address patient 
identified barriers such as lack of time due to employment, transportation issues including travel time and method, or not feeling well enough to attend. Referral to CR programs should consider exploring the accessibility and affordability of CR for participants (such as travel costs, taking time off work, accessing transportation) or referral to social workers to coordinate outpatient resources to support and facilitate access to CR. Strategies may include offering patients alternative, newer models of CR delivery such as home-based programs that have been found to be equally as effective for improving clinical outcomes in recent meta-analyses evaluating home versus center based CR (Dalal et al., 2010). However as noted by Valencia et al., (2011) in a recent review, there has been widespread failure to offer and implement home based programs which they attributed to current $\mathrm{CR}$ funding models for inpatient programs and thus may support policy changes at the provincial and national level. Site-based programs may need to consider offering weekend or evening programs to accommodate younger, employed individuals who are not able to attend CR due to employment or family members who are unable to drive participants during weekday hours. Finally, strategies to decrease wait times from patient referral to intake at CR programs are warranted, as findings from this study identified no patients had yet begun exercise or educational elements of CR programs among those intending to attend CR.

\section{Implications for Education}

Implications for education include a need to address gaps in HCP education related to the management of HF in the outpatient setting through secondary prevention programs. This is important in order to ensure all patients receive education on CR prior to discharge from hospital. In this study, only $50 \%$ of participants had a discussion with their HCP about CR prior to discharge. Among the participants who had a discussion with their HCP about CR, 66.7\% intended to attend CR. In addition, although all patients in this study received an automatic 
referral to CR, only $36.7 \%$ of participants in the study intended to attend CR, suggesting additional strategies other than automatic referral may need to be explored such as endorsement of CR by HCPs in the inpatient setting. Another educational implication includes the need to disseminate study findings through interdisciplinary educational in-services to inform HCPs of the current issues surrounding $\mathrm{HF}$ and $\mathrm{CR}$ attendance in terms of barriers to attendance and identification of patients at high risk of non-attendance. This education may increase the capacity of HCP to identify underserved individuals within the HF population. Incorporating these findings into HCP professional development or during clinical rounds may help to foster interprofessional collaboration in developing strategies to increase CR access and ensure all individuals who are referred, elect to attend. However, it is important to recognize resource constraints (cost of providing professional development education or lack of time among HCPs to provide comprehensive education, resources and endorsement for CR in the busy clinical setting) may have an impact on implementation of strategies to increase CR uptake.

\section{Implications for Policy}

Implications for policy include the development of policies at the organizational level to address the current gap in referral and wait times for CR. Although provincial and national strategic initiatives have provided guidelines for acceptable wait times for CR, the development of policies at the organizational level may be beneficial in ensuring patients engage in CR within 30 days of discharge. Furthermore, policies through the Local Health Integration Networks and at the individual organization level could be extended to include guidelines for expectations and communication between acute care hospitals and CR sites in order to facilitate a systematic and integrated approach to patient follow-up in a timely manner (such as creating standards for time for referral processing, to initial patient follow-up, followed by intake assessment and 
coordinating a CR start date). This responsibility should be shared among acute care institutions and CR facilities. More locally driven policies may serve to increase uptake of CR. Development and uptake of organizational policies and clinical tools would require support and endorsement from organizational leaders, unit managers and educators.

\section{Implications for Theory}

Andersen and Newman's (1973) conceptual framework was valuable in developing the research questions guiding this study as well as providing support for determining which variables to collect and explore. Andersen and Newman identify sex as an important demographic characteristic related to health and illness. For the purpose of this study, the model was modified to explore gender rather than sex as dichotomous views about sex, as being biologically male or female, have shifted since the 1970's when this model was developed (Torgrimson and Minson, 2005). Among the variables explored in this study, findings revealed employment status (working versus not working) was not influencing factor on intention to attend CR. Rather, results revealed time and availability were significant factors influencing intention to attend CR. These results provide implications for future studies, which may consider modification of the current framework to take these factors into consideration when exploring health service use. Among participants who were 'not working' $(\mathrm{n}=20)$ only two participants reported being unemployed, while the majority $(\mathrm{n}=18)$ of participants in this category were retired. Among those intending to attend CR, $81.8 \%$ were retired compared with $47.4 \%$ among those not intending to attend and therefore were significantly more likely to be able to attend CR programs during the day when they are offered.

Future research on health service utilization would benefit from the use of the Health

Care Service Utilization framework for the development of research questions, exploration of 
variables, and interpretation of findings. As this study only focused on some of the individual determinants to health service use, other factors that may impact CR attendance outlined in the literature and supported by Andersen and Newman's (1973) framework should be explored, which include: self-management capacity, self-efficacy, health beliefs, depression, and healthrelated quality of life (Andersen and Emery, 2014; Murray et al., 2012; Reges et al., 2013).

\section{Implications for Research}

With data from 30 participants, findings are unlikely to be generalizable to that of the larger population or in other health care systems where CR service expenses are not covered, requiring patients to pay out of pocket to attend programs. The findings of this study could be used to inform the design of a future study, which could address the lack of participant diversity and underrepresentation of individuals identified in Chapter 2. Specifically, females and various ethnicities were underrepresented in this study with $70 \%$ being males and $76.7 \%$ being Caucasian. This may be due to the prospective design that used convenience sampling for consecutive enrollment of patients upon discharge from hospital, which was required due to the time and resource constraints of this graduate student thesis study. Future studies should consider purposeful sampling or oversampling for women and various ethnicities and for an equal number of participants in each group (attending versus non-attending). A larger sample may demonstrate other socio-demographic or clinical variables to be significant.

This study also demonstrated the feasibility of data collection procedures both in hospital (demographic and clinical data) as well as after hospital discharge (follow-up telephone calls to determine frequency and type of HF-related complications). However, further research on the use of valid and reliable research instruments to assess HF-related complication experiences accurately is needed. Although this study collected complications based on the HF Signs and 
Symptoms Checklist, the psychometric properties for this tool have not been well demonstrated. Few HF-related instruments are available in the literature. Instruments available for assessment of HF symptoms have been adapted from use in other populations, specifically oncology (Memorial Symptom Assessment Scale (MSAS)) or palliative care (Edmonton Symptom Assessment System) or from instruments measuring quality of life (Minnesota Living with HF Questionnaire, Kansas City Living with HF Questionnaire). Other instruments designed for measurement of HF complications assess multiple symptom dimensions (severity and frequency) of both psychological and physical symptoms (HF Symptom Survey, M.D. Anderson Symptom Inventory-HF, MSAS-HF).

Although participants' intention to attend or not attend CR was explored in this study, actual attendance in the CR program was not verified and therefore responses related to intention to attend may not reflect actual attendance. However, the literature supports the validity of $\mathrm{CR}$ self-report data and actual enrollment in CR programs (Kayaniyil, Leung, Suskin, Stewart, \& Grace, 2009).

The findings from this study revealed significant differences in demographic and clinical characteristics of study participants' intention to attend or not attend CR. These findings and the lack of literature on $\mathrm{CR}$ as a secondary prevention program in the HF population supports the need for additional research on patient characteristics associated with attendance or nonattendance at CR in this population. This study analyzed data collected over a 16-week period collection period. A prospective study that recruits patients over a longer period of time, such as 12 months, and recruiting from multiple institutions would be valuable in identifying attendance patterns at $\mathrm{CR}$ among individuals with $\mathrm{HF}$ and further validate the findings in this study. Future research into strategies to improve $\mathrm{CR}$ attendance among those who do not intend to attend $\mathrm{CR}$ 
and the effectiveness of these strategies is recommended. Specifically, strategies to mitigate some of the modifiable barriers identified by patients in this study include: improving patient education and HCP education to include the importance of HCP endorsement of CR, providing HF patients with information about the benefits and reason for referral to CR prior to discharge from the hospital setting; assessing patient transportation issues; facilitating CR program planning that responds to patient preferences for the time $\mathrm{CR}$ is offered so that $\mathrm{CR}$ programs can accommodate employed individuals and others who may prefer an evening or weekend program; and developing and evaluating different models of CR delivery such as home-based CR. Additionally, future studies should explore clinical outcomes of attendance versus nonattendance at $\mathrm{CR}$ of patients with $\mathrm{HF}$ due to the limited research available with this population specifically.

\section{Feasibility of Study Design and Procedures}

Implications for feasibility include an evaluation of the methodological components of this study: study design, sample size, participant recruitment and data collection instruments and procedures. The choice of study design was appropriate to describe socio-demographic and clinical characteristics associated with intention to attend CR in the HF population. Based on the results of this study, participants fell into one of two groups: intention to attend CR and no intention to attend CR. A future study using a comparative descriptive design would ensure an equal number of participants in both groups. The aim for a sample size of 30 participants in this

study was appropriate based on recommendations in the literature while accounting for potential participant withdrawal and a single researcher for recruitment. A total of 30 participants were successfully recruited over a 16-week data collection period. Factors associated with successful recruitment in this study included the researcher connecting with the unit managers, patient care 
coordinators and staff nurses prior to beginning the study to introduce the study as well as become familiar with unit-specific procedures to determine how best to recruit patients in consultation with unit staff. The researcher also attended clinical rounds on a daily basis from Monday to Friday to connect with members of the interprofessional team to introduce the study and identify eligible participants. Establishing good rapport and communication with the interprofessional team members and unit patient care coordinators was very important for successful identification of eligible participants and subsequent recruitment by the researcher. In addition, the timing of recruitment was important in this study. Patients were often approached in the late afternoon or early evening, which are quieter times in the hospital setting for patients. The researcher ensured timely collection of socio-demographic data by reading the questionnaire to participants during the initial enrollment visit. The findings of this study suggest the data collection surveys are feasible and useful measures for data collection. Future studies may consider further use and validation of these tools.

\section{Dissemination of Findings}

The study findings will be disseminated through a publication of this thesis in peerreviewed journals. A final report summarizing study findings will also be prepared and disseminated upon request to study participants or other individuals who express interest in study findings. Results of the study will also be disseminated to the institutions at which the study took place through presentations to unit staff and organization wide research conferences. Further dissemination strategies include submission of abstracts to various appropriate conferences or webinar presentations at the national level (e.g. Canadian Council of Cardiovascular Nurses Conference, Canadian Cardiovascular Congress, Canadian Association of Cardiovascular Rehabilitation and Prevention Conference). 


\section{Summary}

While additional research is required, this descriptive pilot study provides empirical evidence of feasibility for the design of a larger study, identified patients with HF who are at risk for CR non-attendance, as well as highlighting the complications experienced by individuals after discharge from hospital while awaiting intake into CR. Findings from the study highlight the need for targeted care based on collection of socio-demographic and clinical differences in patients with HF, and the need for HCPs to develop population-specific strategies aimed at increasing CR attendance by individuals with $\mathrm{HF}$. Improving how we care for patients with $\mathrm{HF}$ requires hospital to examine how they identify, refer and support intention to attend $\mathrm{CR}$ and refer HF patients for CR. Recommendations for CR programs include exploring other models of CR, providing $\mathrm{CR}$ at times and days that meet the needs of all patients who could benefit, and addressing wait times for CR given the clinical complications patients experience while waiting. Study findings add to the existing literature into factors influencing intention to attend CR in an underrepresented cardiac population in the CR literature. 


\section{Appendix A}

Participant \#:

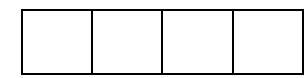

Week \# (circle): $1 \quad 3 \quad 5 \quad 6$

\section{Heart Failure Related Complications Survey Data Collection Form}

Place a check mark in the box if patient answers "yes" to any of the following complications:

$\square$ Shortness of breath (dyspnea)

Lack of energy (fatigue)

Difficulty sleeping

Difficulty breathing when lying flat (orthopnea)

$\square$ Waking up breathless at night (paroxysmal nocturnal dyspnea)

$\square$ Swelling of legs or ankles (edema)

$\square$ Dizziness

$\square$ Chest discomfort

$\square$ Palpitations

$\square$ Lack of appetite

Decreased ability to exercise or carry out activities (exercise intolerance)

$\square$ Change in urine output compared with normal (darker color, voiding less often or in small amounts)

$\square$ Sudden Weight Gain

$\square$ Weight Loss

$\square$ Severe cough that keeps you awake at night or causes chest pain

(Weeks 1 and 6)

Is the participant planning on attending cardiac rehabilitation?

$\square$ Yes $\quad \square$ No

If no, reason(s) why participant has chosen not to attend cardiac rehabilitation:

(Week 6 Only)

Has the participant receive a phone call for a start date for cardiac rehabilitation?

$\square$ Yes $\quad \square$ No

If yes, scheduled start date is: 


\section{Appendix B}

\section{Introduction of Study Script for Patient Circle of Care Member}

"I would like to tell you about a research study that you may be interested in. The study is recruiting patients who have heart failure and who are or will be referred to a cardiac rehabilitation program after they are discharged from the hospital.

If you are interested in hearing more about this study, may I have your permission to have the nurse researcher speak to you about this study? " 


\section{Appendix C}

\section{Recruitment Script For Study Investigator In-Person Contact}

"Hello, my name is Kristen Cunningham and I am working on a research study as part of my Master of Nursing program at Ryerson University. “

"You were informed of this study by one of the health care professionals involved in your care and you indicated you would be interested in learning more about the study. Is it OK if I continue?"

"I am providing you with a study information letter which I can review with you and answer any questions you may have."

"You are being invited to attend in this study because you have a diagnosis of heart failure and your physician or nurse practitioner has referred you to a cardiac rehabilitation program."

"The purpose of this study is to collect information about patients who are referred to cardiac rehabilitation and if they plan to attend or not attend the program. The secondary purpose of this student is to identify any heart failure related complications experienced by patients once they are discharged from hospital and while they await intake into a cardiac rehabilitation program up to 6 weeks after hospital discharge."

"Information learned from this study may help to inform health care professionals of patients who do and do not attend cardiac rehabilitation for heart failure so strategies can be identified to increase patient participation in cardiac rehabilitation programs in the future."

Researcher to review all details of the study outlined in the information letter with patient and will answer any questions the patient may have.

"Would you be interested in participating in this study?"

If patient answers "yes" - move on to consent form

If patient answers "no" - thank patient for their time and do not continue 


\section{Appendix D \\ Letter of Information: Research Study}

Title: Demographic Characteristics of Patient Participation in Cardiac Rehabilitation Following Hospitalization for Heart Failure

\section{Background}

If you have a diagnosis of heart failure and have been referred to cardiac rehabilitation, you are invited to voluntarily attend in a research study directed by Ms. Kristen Cunningham as part of the Master of Nursing thesis requirements for Ryerson University. There are two related purposes for doing this study. The first is to identify characteristics of individuals living with heart failure who are referred to cardiac rehabilitation and plan to attend or not attend the program. This study will also look at the number and type of complications experienced by those living with heart failure over a 6-week time period after discharge from hospital.

\section{Details of the Study}

If you choose to attend, you will be asked to answer a questionnaire while in the hospital. The questionnaire will ask information about: age, sex, race, relationship status, employment status, income level, who you live with, education level, family supports, access to transportation, heart failure classification and other health conditions you may be living with.

Kristen Cunningham, the researcher, will telephone you four times at week 1,3,5, and 6 after you leave the hospital and ask information about:

i. Whether or not you have experienced heart failure complications since leaving the hospital ii. Whether or not you plan to attend cardiac rehabilitation and received a call for a start date

Completing the questionnaire and responding to each telephone call will take about 10-20 minutes.

\section{Other Information}

Participation in this study is voluntary. Your decision to attend or not will not have an effect upon your care. If you are interested in participating in this study, please let the researcher know and the researcher will go over the consent form with you and answer any questions you may have. If you would like to think about participation before making a decision, you may contact Kristen Cunningham at: $\mathrm{xxx}-\mathrm{xxx}-\mathrm{xxxx}$ within one week if you decide to attend. If you would like more information about the study please call Kristen Cunningham at xxx-Xxxxxxx or Helen Kelly at xxx-Xxx-Xxxx ext.xxxx

Sincerely,

Kristen Cunningham, RN, Master of Nursing Thesis Student, Ryerson University 


\section{Appendix E \\ CONSENT FORM TO ATTEND IN A RESEARCH STUDY}

Study Title:

Principal Investigator:

Co-Investigator:
Demographic Characteristics of Participation in Cardiac Rehabilitation Following Hospitalization for Heart Failure

Helen Kelly (xxx-xxx-xxxx xxxx)

Kristen Cunningham, RN (xxx-xxx-xxxx)

E-mail: kcunningham@ryerson.ca

\section{Email Disclaimer}

Please note that the security of email is not guaranteed. Messages may be forged, forwarded or kept indefinitely by others using the internet. Do not use the e-mail to discuss information you think is sensitive. Do not use e-mail in an emergency since email may be delayed.

\section{Introduction:}

You are being invited to take part in a research study. This research study will fulfill the requirements of a Master Thesis in Nursing for Kristen Cunningham at ... institution name.... Please read the information about the study presented in this form. The form includes details on study's risks and benefits that you should know before you decide if you would like to take part. You should take as much time as you need to make your decision. You should ask the principal investigator or study staff to explain anything that you do not understand and make sure that all of your questions have been answered before signing this consent form. Before you make your decision, feel free to talk about this study with anyone you wish including your friends, family, and family doctor. Participation in this study is voluntary.

\section{Background/Purpose:}

You are being invited to voluntarily attend in this study because you have a diagnosis of heart failure and have been referred to a cardiac rehabilitation program.

There are two related purposes to doing this study. First we would like to examine characteristics of people with heart failure who are referred to a cardiac rehabilitation program and plan to attend the program or not attend the program. We will also look at the number and type of complications experienced by those living with heart failure who attend in a cardiac rehabilitation program compared to those who do not attend Up to 30 people at Toronto General Hospital will attend in this study and it will take 6 weeks to complete.

\section{Study Visits and Procedures:}

Your voluntary participation in this study will involve filling out a questionnaire before you leave the hospital and answering questions over the phone at weeks 1, 3, 5, and 6 
after you leave the hospital. Each contact with the researcher will take about $10-20$ minutes.

The questionnaire you will fill out prior to leaving the hospital will include questions about: age, sex, race, education level, employment status, income level, relationship status, family supports, living arrangement, transportation, other health conditions, classification of heart failure, contact information (phone number, city lived in, postal code) in order for the researcher to follow-up with you by telephone and determine the distance you live from a cardiac rehabilitation facility.

The researcher will look at your medical records for the purpose of identifying any other health conditions you may be living with and your New York Heart Association functional classification, which is used to identify how severe your heart failure is based on how much you are limited during physical activity.

The telephone calls at weeks $1,3,5$ and 6 will include questions about any heart failure related complications you have experienced at home, for example, shortness of breath, sudden weight gain or swelling of the legs and ankles. If you do not feel comfortable answering any particular question you simply note that you do not wish to answer.

During the telephone call at 1 week you will be asked if you are planning to attend a cardiac rehabilitation program.

During the telephone call at 6 weeks you will also be asked if you are planning to attend a cardiac rehabilitation program and if you have received a phone call with a start date for the program. If you are planning not to attend the program, the researcher will ask about why you have chosen not to attend.

If we happen to call you at an inconvenient time, please note this and we will find a mutually convenient time to contact you during that week.

\section{Summary of Procedures:}

\begin{tabular}{|l|l|l|}
\hline $\begin{array}{l}\text { Time Points for } \\
\text { Data Collection }\end{array}$ & Information To Be Collected & Method of Data Collection \\
\hline $\begin{array}{l}\text { In-hospital, } \\
\text { following consent } \\
\text { of participant }\end{array}$ & $\begin{array}{l}\text { Demographic Questionnaire: age, } \\
\text { gender, race, education level, } \\
\text { employment status, income level, } \\
\text { relationship status, family supports, } \\
\text { living arrangements, other health } \\
\text { conditions, classification of heart } \\
\text { failure }\end{array}$ & $\begin{array}{l}\text { Questionnaire - a } \\
\text { researcher will ask the } \\
\text { questions and fill out the } \\
\text { questionnaire }\end{array}$ \\
& $\begin{array}{l}\text { Participant contact information } \\
\text { (telephone number, city lived in, }\end{array}$ & \\
\hline
\end{tabular}




\begin{tabular}{|c|c|c|}
\hline & postal code) & \\
\hline \begin{tabular}{|l|} 
Week 1 \\
post- discharge
\end{tabular} & $\begin{array}{l}\text { Heart Failure-related complications } \\
\text { Participants will be asked if they are } \\
\text { planning on attending cardiac } \\
\text { rehabilitation. } \\
\text { If a participant is not planning to } \\
\text { attend cardiac rehabilitation, the } \\
\text { researcher will ask the participant } \\
\text { why they have chosen not to attend. }\end{array}$ & Telephone Call \\
\hline $\begin{array}{l}\text { Week } 3 \\
\text { post-discharge }\end{array}$ & Heart Failure-related complications & Telephone Call \\
\hline $\begin{array}{l}\text { Week } 5 \\
\text { post-discharge }\end{array}$ & Heart Failure-related complications & Telephone Call \\
\hline $\begin{array}{l}\text { Week } 6 \\
\text { post-discharge }\end{array}$ & $\begin{array}{l}\text { Heart Failure-related complications } \\
\text { If a participant has received a phone } \\
\text { call for a start date at cardiac } \\
\text { rehabilitation and is planning to } \\
\text { attend the program. } \\
\text { If participant is not planning to attend } \\
\text { cardiac rehabilitation, the researcher } \\
\text { will ask why they have chosen not to } \\
\text { attend. }\end{array}$ & Telephone Call \\
\hline
\end{tabular}

\section{Risks:}

There are no known physical risks associated with participation in the study.

Please remember you will be asked about any heart failure-related complications you may be experiencing for the purpose of research. If you are experiencing any of these symptoms the researcher will be sure to remind you to follow-up with your health care provider or seek emergency care and report to the principal investigator of this study.

Being in this study may make you feel uncomfortable, anxious, or distressed as you will be talking about your diagnosis of heart failure and your health state. You may refuse to answer questions or stop the interview at any time for any reasons.

\section{Benefits:}

You may not receive any direct benefit from being in this study. We hope that information learned from this study may help health professionals identify strategies to increase heart failure patient participation in cardiac rehabilitation programs in the future. 


\section{Voluntary Participation:}

Your participation in this study is voluntary. You may decide not to be in this study, or to be in the study now and then change your mind later. You may leave the study at any time without affecting your care in any way. Your health care providers will not be informed of your decision to attend or not attend a cardiac rehabilitation program.

\section{Confidentiality:}

If you agree to join this study, the principal investigator and his/her study team will look at your personal health information and collect only the information needed for the purposes of this study. Personal health information is any information that could be used to identify you and includes your:

- Name

- Address

- Postal Code

- Date of birth

- New or existing medical records, that includes types, dates and results of medical tests or procedures

For the purpose of this study, your name and postal code will be collected if you decide to attend. The researcher will ask you to provide your postal code during the first enrollment visit where you will be asked to complete a written questionnaire with the researcher.

All information collected during this study, including your personal health information, will be securely stored at...institution name... in a locked filing cabinet and on an encrypted USB. The information will also be stored on a secure server which is password protected. The information will not be shared with anyone outside the study unless required by law. In addition to the lead researchers, the following people may come to the hospital to look at the study records and at your personal health information to check that the information collected for the study is correct and to make sure the study is following proper laws and guidelines: Representatives of...institution name....including the....institution name... Research Ethics Board and...institution name...Research Ethics Board.

The principal investigator will keep any personal health information about you in a secure and confidential location for 10 years. All information will be shredded after 10 years.

A list linking your study number with your name will be kept by the study investigators in a secure place, separate from your study file. Consent forms will be kept separate from 
all other data forms.

You will not be named in any reports, publications, or presentations that may come from this study.

If you decide to withdraw from the study, the information that was collected before you left the study will still be used in order to help answer the research question. No new information will be collected without your permission.

\section{Costs and Reimbursement:}

You will not have to pay for any of the procedures involved with this study and will not incur any expenses as a result of your participation in the study. You will not be reimbursed for time or inconvenience.

\section{Rights as a Participant:}

If you are harmed as a direct result of taking part in this study, all necessary medical treatment will be made available to you at no cost.

By signing this form you do not give up any of your legal rights against the investigators, sponsor or involved institutions for compensation, nor does this form relieve the investigators, sponsor or involved institutions of their legal and professional responsibilities.

\section{Conflict of Interest:}

The investigators conducting this study have a personal and professional interest in completing this study. Their interests should not influence your decision to attend in this study. You should not feel pressured to join this study.

\section{Questions about the Study:}

If you have any questions, concerns or would like to speak to the study team for any reason, please call: Kristen Cunningham, RN at xxx-xxx-xxxx, Helen Kelly at xxx-xxx$x x x x$ ext. $x x x x$ or Dr. Suzanne Fredericks at $x x x x-x x x x-x x x x$ ext. $x x x x$.

If you have any questions about your rights as a research participant or have concerns about this study, call the Chair of the...institution name... Research Ethics Board (....institution name...REB) or the Research Ethics office number at xxx-xxx-xxxx. You can also contact the Chair of the Human Research Ethics Board at...institution name....at xxx-xxx-xxxx ext. xxxx or by email at rebchair@.ca. The REB is a group of people who oversee the ethical conduct of research studies. The...institution name... and...institution name... REB is not part of the study team. Everything that you discuss will be kept confidential.

You will be given a signed copy of this consent form. 


\section{Dissemination of findings:}

A copy of the final report will be made available to you either electronically or by hard copy upon request. Please contact Kristen Cunningham, RN at XXX-XXX-XXXX.

\section{Consent:}

This study has been explained to me and any questions I had have been answered.

I know that I may leave the study at any time. I agree to the use of my information as described in this form. I agree to take part in this study.

Print Study Participant's Name

Signature

Date

My signature means that I have explained the study to the participant named above. I have answered all questions.

Print Name of Person Obtaining Consent Signature

Date 


\section{Appendix F}

Participant Study \#:

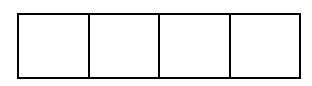

Date of Completion $(\mathrm{mm} / \mathrm{dd} / \mathrm{yy})$ :

Demographic Questionnaire

(1 of 3$)$

The following personal information is being collected for the purpose of this research study. This information is being kept completely confidential and will be stored in a secure area, which only the lead researchers have access to. For each question, choose only ONE answer unless otherwise indicated.

Remember that you can choose not to answer any particular question and still complete the questionnaire.

1. What is your age in years?

2. What is your sex? Male ( ) Female ( ) Other ( )

3. What ethnicity do you consider yourself to be?

4. What is your present relationship status?
( ) 1. Single
( ) 2. Married
( ) 3. Divorced
( ) 4. Separated
( ) 5. Widowed
( ) 6. Common-Law
( ). 7 Other:

5. What is your employment status?
( ) 1. Employed
( ) 2. Unemployed
( ) 3. Retired
( ) 4. Volunteer

5 a. If employed do you work: Full-time ( ) Part-time ( ) Casual ( )

6. What is your average personal annual income?

( ) 1. less than $\$ 30,000$

( ) 2. $\$ 30,001-40,000$

( ) 3. $\$ 40,001-50,000$

( ) 4. $\$ 50,001-60,000$

( ) $5 . \$ 60,001-70,000$

( ) $6 . \$ 70,001-80,000$

( ) 7. Greater than 80,000

( ). Prefer not to say 
Participant Study \#:

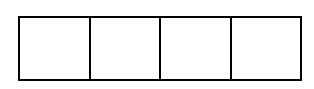

\section{Demographic Questionnaire ( 2 of 3$)$}

7. Who do you live with?
( ) 1 . Alone
( ) 2. Spouse / Common-Law
( ) 3. Family / Friends
( ) 4. Other (please specify:

8. What is the highest level of education you have completed?
( ) 1 . None
( ) 2. Elementary school
( ) 3. High school
( ) 4. College
( ) 5. Undergraduate university
( ) 6. Professional degree
( ) 7. Graduate degree

8. Do you have friends or family you can rely on if you need help or support? ( ) 1. Yes ( ) 2. No

9. Do you have access to transportation to attend cardiac rehabilitation? $\quad($ ) 1. Yes ( ) 2. No

9. A) What type of transportation will you use to get to cardiac rehabilitation?

( ) Driving self

( ) Family member / Friend Driving

( ) Volunteer Driver

( ) Taxi

( ) Public Transit

( ) Other

10. Did someone speak to you about the cardiac rehabilitation facility you are being referred to?

(1) Yes

(2) No

If yes, approximately how long will it take you to get to the cardiac rehabilitation facility?

(1) 30 minutes or less

(2) 45 minutes

(3) 1 hour

(4) Over 1 hour 
Participant Study \#:

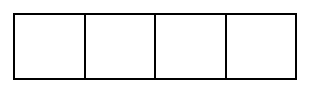

\section{Demographic Questionnaire}

(2 of 3$)$

If no, please provide postal code (to determine the distance the patient lives from the cardiac rehabilitation center closest to their home, which is where they will automatically be referred):

The researcher will refer to the patient discharge summary, located in the patient chart for questions 11 and 12 .

11. New York Heat Association Classification of Heart Failure:
( ) $1 . \mathrm{I}$
( ) 2. II
( ) 3 . III
( ) 4. III

12. Other current health conditions (check all that apply):
( ) Cardiac
( ) Respiratory
( ) Musculoskeletal
( ) Other: 


\section{Appendix G}

Participant Study \#:

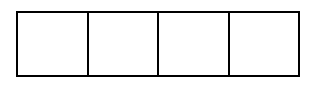

\section{Patient Contact Form}

CONFIDENTIAL: Retain separately from participant file

Date of Participant Consent to Attend (mm/dd/yy):

Patient's Contact Information:

Name (last name, first name):

Telephone \#:

Preferred time and day to contact:

( ) Morning

( ) Afternoon

( ) Evening

( ) Monday

( ) Tuesday

( ) Wedneday

( ) Thursday

( ) Friday

( ) Saturday

( ) Sunday

If the participant is unable to answer the phone call, the researcher may leave a message:

( ) Yes

( ) No 


\section{Appendix H \\ Telephone Call Script: Weeks 1,3,5, 6}

Follow-up telephone calls will be delivered to participants at weeks 1, 3, 5, and 6 postdischarge from hospital for a total of 4 follow-up calls.

\section{Telephone Call Weeks 1, 3, 5, 6}

The researcher will first re-introduce herself and the purpose of the study to the participant.

"Hello ___ (state participant name). My name is Kristen Cunningham. I am calling to followup with you because you consented to attend in the heart failure research study before you were discharged from hospital. I'm calling today to talk with you for about 10 minutes if and first just want to confirm that you are still willing to attend?"

If participant says no, researcher to first ask "Can I call back at a different time that is more convenient for you?"

If no, thank participant for their time and do not continue phone call.

If yes, "is this a good time to talk?"

If no, the researcher will arrange for another mutually convenient time.

If yes, continue with script.

"Today I am going to talk to you about whether or not you have had any complications in the past__ weeks (state number of weeks patient has been discharged from hospital). I am going to list a number of possible complications that some people with heart failure may have. These questions are easy to answer simply with "yes" or "no" however please feel free to discuss your experiences of these symptoms with me if you wish to do so. If you do not feel comfortable answering any question you can ask to skip the question and do not have to answer. If at any time you would like to discontinue the call please just let me know.

Do you have any questions before I continue?”

If yes, then take time to answer the participant's questions.

If no, continue with script. 
"In the past weeks (state number of weeks patient has been discharged from hospital), have you experienced:

Shortness of breath

Lack of energy

Difficulty sleeping

Difficulty breathing when lying flat

Waking up breathless at night

Swelling of legs or ankles

Dizziness

Chest discomfort

Palpitations

Lack of appetite

Decreased ability to exercise or carry out activities

Change in urine output compared with normal, such as darker color, voiding less often or in small amounts

Sudden Weight Gain

Weight Loss

Severe cough that keeps you awake at night or causes chest pain "

\section{Week 1 Additional Question}

"Are you planning to attend a cardiac rehabilitation program?"

$\square$ Yes $\quad \square$ No

\section{Week 6 Additional Questions}

"Are you planning to attend a cardiac rehabilitation program?"

$\square$ Yes $\quad \square$ No

If no, "are there any reasons why you have chosen not to attend?"

"Have you received a phone call for a star date for cardiac rehabilitation over the past 6 weeks?"

$\square$ Yes $\quad \square$ No

If yes, "what is the start date of the cardiac rehabilitation program?" 


\section{Closing Script For Telephone Calls}

"Well___ (state participant's name), those are all of the questions I have for today. Do you have any other questions I can answer for you?"

If yes, answer questions.

If no, continue with script.

"I would like to thank you for your time today and remind you I will be calling you in the next week (state number of weeks) on (state date) at (state time

previously discussed with participant during the enrollment visit, on patient contact form)"

"Does this date and time work for you?"

If yes, thank participant again for their time and finish phone call.

If no, arrange for another mutually convenient time, thank participant again for their time and end phone call.

\section{Script for Voicemail Message}

"Hello, this message is for (state participants name). This is Kristen Cunningham calling. I will try to reach you at another time or you can call me back at 437-350-7673 if you would like. Thank you" 


\section{References}

Ades, P. (2015). Temporal trends and factors associated with cardiac rehabilitation referral among patients hospitalized with heart failure. Journal of the American College of Cardiology, 66(8). doi:10.1016/j.jacc.201506.1090

Ades, P., Keteyian, S., Balady, G., Houston-Miller, N., Kitzman, D., Mancini, D., \& Rich, M. (2013). Cardiac rehabilitation exercise and self-care for chronic heart failure. JACC: Heart Failure, 1(6). doi:10.1016/j.jchf.2013.09.002

Albert, N., Trochelman, K., Li, J., \& Lin, S. (2010). Signs and symptoms of heart failure: are you asking the right questions? American Journal of Critical Care, 19(5), 443-452. doi: 10.4037/ajcc2009314

Albert, N., Fonarow, G., Abraham, W., Chiswell, K., Gattis-Stough, W., Gheorghiade, M.,... Young, J. (2007). Predictors of Delivery of Hospital-Based Heart Failure Patient Education: A Report from OPTIMIZE-HF. Journal of Cardiac Failure, 13(3). doi: http://dx.doi.org/10.1016/j.cardfail.2006.11.008

Almerud-Osterbeg, S., Baigi, A., Bering, C., \& Fridlund, B. (2014). Knowledge and beliefs about risk factor information among non-attendees in cardiac rehabilitation programs. Nordic Journal of Nursing Research, 99(31), 29-33. Retrieved from http://journals.sagepub.com/doi/pdf/10.1177/010740831103100107

Alves, A., Ribeiro, F., Goldhammer, E., Rivlin, Y., Rosenschein, U., Viana, J.,...Oliveria, J.(2012) Exercise training improves diastolic function in heart failure patients. Medicine \& Science in Sports \& Exercise, 44(5), 776-785. doi: 10.1249/MSS.0b013e31823cd16a 
American Heart Association. (2015). Understand your risks for heart failure. Retrieved from

http://www.heart.org/HEARTORG/Conditions/HeartFailure/UnderstandYourRiskforHear tFailure/Understand-Your-Risks-for-Heart-Failure_UCM_477645_Article.jsp

American Heart Association. (2015). Causes and risks for heart failure. Retrieved from http://www.heart.org/HEARTORG/Conditions/HeartFailure/UnderstandYourRiskforHear tFailure/Causes-and-Risks-for-Heart-Failure_UCM_002046_Article.jsp

Andersen, R.M., \& Newman, J.F. (1973). Societal and individual determinants of medical care utilization in the United States. Milbank Memorial Fund Quarterly-Health and Society, 51(1), 95-124.

Andersen, D., \& Emery, C. (2014). Irrational health beliefs predict adherence to cardiac rehabilitation: A pilot study. Health Psychology, 33(12), 1614-1617. doi:10.1037/hea0000017

Angus, J.E., King-Shier, K.M., Spaling, M.A., Duncan, A.S., Jaglal, S.B., Stone, J.A., \& Clark, A.M. (2015). A secondary meta-synthesis of qualitative studies of gender and access to cardiac rehabilitation. Journal of Advanced Nursing, 71(8), 1758-1773.

doi: $10.1111 /$ jan.12620

Arena R., Williams M., Forman D.E., Cahalin, L.P., Coke, L., Myers, J.,... Lavie, C.J. (2012). Increasing referral and participation rates to outpatient cardiac rehabilitation: the valuable role of healthcare professionals in the inpatient and home health settings: a science advisory from the American Heart Association. Circulation,125(10), 1321-1329. 10.1161/CIR.0b013e318246b1e5 
Armstrong, A., Martin, B.J., Arena, R., Hauer, T., Austford, L., Stone, J.,... Signal, R. (2014).

Patients with diabetes in cardiac rehabilitation: Attendance and exercise capacity. Medicine \& Science in Sports \& Exercise, 46(5), 845-850. doi:

10.1249/MSS.0000000000000189

Arnold, J., Liu, P., Demers, C., Dorian, P., Giannetti, N.,...White, M. (2006). Canadian Cardiovascular Society consensus conference recommendations on heart failure 2006: Diagnosis and management. Canadian Journal of Cardiology, 22 (1), 23-45. http://www.ccs.ca/images/Guidelines/Guidelines_POS_Library/HF_CC_2006.pdf

Baranson, S., Zimmerman, L., \& Young, L. (2012). An integrative review of interventions promoting self-care of patients with heart failure. Journal of Clinical Nursing, 21(3-4), 448-475. doi: 10.1111/j.1365-2702.2011.03907.x

Belardinelli, R., Georgiou, D., Cianci, G., \& Purcaro, A. (2012). 10-year exercise training in chronic heart failure: a randomized controlled trial. Journal of the American College of Cardiology, 60(16), 1521-1528. doi: 10.1016/j.jacc.2012.06.036

Blair, J., Angus, N., Lauder, W., Atherton, I., Evans, J., \& Leslie, S. (2014). The influence of non-modifiable illness perceptions on attendance at cardiac rehabilitation. European Journal of Cardiovascular Nursing, 13(1) 55-62. doi: 10.1177/1474515113477272.

Browne, R.H., (1995). On the use of a pilot sample for sample size determination. Statistics in Medicine, 14(17), 1933-1940

Brual, J., Gravely-Witte, S., Suskin, N., Stewart, D., Macpherson, A., \& Grace, L. (2010). Drive time to cardiac rehabilitation: at what point does it affect utilization? International Journal of Health Geographics, 9(27). doi: 10.1186/1476-072X-9-27

Bui, A., Horwich, T., \& Fonarow, G. (2011). Epidemiology and risk profile of heart 
failure. Nature Reviews Cardiology, 8(1), 30-41. doi: 10.1038/nrcardio.2010.165

Buttery, A.K., Carr-White, G., Martin, F.C., Glaser, K., \& Lowton, K. (2014). Cardiac rehabilitation for heart failure: Do older people want to attend and are they referred? European Geriatric Medicine, 5, 246-251. doi:10.1016/j.eurger.2014.04.011

Canadian Institutes of Health Research, Natural Sciences and Engineering Research Council of Canada, and Social Sciences and Humanities Research Council of Canada. (2014). TriCouncil Policy Statement: Ethical Conduct for Research Involving Humans. Retrieved from http://www.pre.ethics.gc.ca/pdf/eng/tcps2-2014/TCPS_2_FINAL_Web.pdf

Canadian Institute for Health Information. (2015). Reasons for inpatient hospitalization and surgery vary across Canada. Retrieved from https://www.cihi.ca/en/summ_inpatient_hosp_en.pdf

Canadian Institute for Health Information. (2012). All-Cause Readmission to Acute Care and Return to the Emergency Department. Retrieved from, https://secure.cihi.ca/free_products/pReadmission_to_acutecare_en.pdf

Clark, A.M., King-Sheir, K.M., Spaling, M.A., Duncan, A.S., Stone, J.A., Jaglal, S.B., ...Angus, J.E. (2013). Factors influencing participation in cardiac rehabilitation programmes after referral and initial attendance: qualitative systematic review and meta-synthesis. Clinical Rehabilitation, 27(10), 948-959. doi: 10.1177/0269215513481046

Commodore-Mensah, Y., \& Dennison Himmelfarb, C. (2012). Patient education strategies for hospitalized cardiovascular patients: A systematic review, Journal of Cardiovascular Nursing, 27(2), 154-174. Retrieved from, http://www.nursingcenter.com/journalarticle?Article_ID=1302796\#P57

Corra, U., Giannuzzi, P., Adamopoulos, S., Bjornstad, H., Bjarnason-Weherns, B., 
Cohen-Solal, A.,...Vanhees, L. (2005). Executive summary of the position paper of the working group of cardiac rehabilitation and exercise physiology of the European society of cardiology (ESC): Core components of cardiac rehabilitation in chronic heart failure. European Society of Cardiology, 12(4), 321-325.

Dai, S., Walsh, P., Wielgosz, A., Gurevich, Y., Bancei, C., \& Morrison, H. (2012).

Comorbidities and mortality associated with hospitalized heart failure in Canada. Canadian Journal of Cardiology, 28(1), 74-79. doi: 10.1016/j.cjca.2011.05.002

Dafoe, W., Arthur, H., Stokes, H., Morrin, L., \& Beaton, L. (2006). Universal access: But when? Treating the right patient at the right time: Access to cardiac rehabilitation. Canadian Journal of Cardiology, 22(11), 905-911. Retrieved from https://www.ncbi.nlm.nih.gov/pmc/articles/PMC2570237/

Dalal, H., Zawada, A., Jolly, K., Moxham, T., \& Taylor, R. (2010). Home based versus centre based cardiac rehabilitation: Cochrane systematic review and meta-analysis. British Medical Journal, 340:b5631. doi: 10.1136/bmj.b5631

Davidson, P., Cockburn, J., Newton, P., Webster, J., Betihavas, V., Howes, L., Owensby, D. (2010). Can a heart failure-specific cardiac rehabilitation program decrease hospitalizations and improve outcomes in high-risk patients? European Journal of Preventive Cardiology, 17(4), 393-402. doi: 10.1097/HJR/0b013e328334ea56

Davies, E., Moxham, T., Rees, K., Singh, S., Coats, A., Ebrahim, S.,...Taylor, R. (2010). Exercise training for systolic heart failure: Cochrane systematic review and metaanalysis. European Journal of Heart Failure, 12(7), 706-715. doi: 10.1093/eurjhf/hfq056 de Melo Ghisi, G.L., Oh, P., Benetti, M., \& Grace, S.L. (2013). Barriers to cardiac rehabilitation 
use in Canada versus Brazil. Journal of Cardiopulmonary Rehabilitation and Prevention, 33(3), 173-179. doi: 10.1097/HCR.0b013e3182930c9f

Desai, A., \& Stevenson, L. (2012). Rehospitalization for heart failure: Predict or prevent? Circulation, 126, 501-506. doi: 10.1161/CIRCULATIONAHA.112.125435

Doll, J., Hellkamp, A., Thomas, L., Ho, M., Kontos, M., Whooley, M.,...Peterson, E. (2015). Effectiveness of cardiac rehabilitation among older patients after acute myocardial infarction. American Heart Journal, 170(5), 855-864. doi: 10.1016/j.ahj.2015.08.001

Dunlay, S., \& Roger, V. (2014). Understanding the epidemic of heart failure: Past, present, and future. Current Heart Failure Reports, 11, 404-415. doi: 10.1007/s11897014-0220-x

Dunlay, S.M., Witt, B.J., Allison, T.G., Hayes, S.N., Weston, S.A., Keopsell, E., \& Roger, V.L. (2009). Barriers to participation in cardiac rehabilitation. American Heart Journal, 148(5), 852-859. doi: 10.1016/j.ahj.2009.08.010

Employment and Social Development Canada. (2015). Canadians in context-Aging population. Retrieved from, http://well-being.esdc.gc.ca/misme-iowb/.3ndic.1t.4r@eng.jsp?iid $=33$

Endo, N., Goto, A., Suzuki, T., Matsuda, S., \& Yasumura, S. (2015). Factors associated with enrollment and adherence in outpatient cardiac rehabilitation in Japan. Journal of Cardiopulmonary Rehabilitation and Prevention, 35(3), 186-192. doi: 10.1097/HCR.0000000000000103

Flynn, K., Pina, I., Whellan, D., Lin, L., Blumenthal, J., Ellis, S.,...Weinfurt, K. (2009). Effects of exercise training on health status in patients with chronic heart failure: Findings from 
the HF-ACTION randomized controlled trial. JAMA, 301(14), 1451-1459.

doi: $10.1001 /$ jama.2009.457

Forman, D.E., Sanderson, B.K., Josephson, R.A., Raikhelkar, J., \& Bittner, V. (2015). Heart failure as a newly approved diagnosis for cardiac rehabilitation: Challenges and opportunities. Journal of the American College of Cardiology, 65(24), 2652-2659. doi: $10.1016 /$ j.jacc.2015.04.052

Ghanbari-Firoozabadi, M., Rahimianfar, A.A.,Nasab, M., Mamayandeh, S.M., Emami, M., Boostani, F.,...Barzegar, K. (2014). A study of the effect of cardiac rehabilitation on heart failure patients' life quality. Journal of Medicine and Life, 7(1), 51-54. Retrieved from, http://www.ncbi.nlm.nih.gov/pmc/articles/PMC3956097/

Golwala, H., Pandey, A., Ju, C., Butler, J., Yancy, C., Bhatt, D.,...Fonarow, G. (2015). Temporal trends and factors associated with cardiac rehabilitation referral among patients hospitalized with heart failure. Journal of the American College of Cardiology, 66(8). doi: 10.1016/I.jacc.2015.06

Grace, S., Oh, P., Marzolini, S., Collela, T., Tan, Y., \& Atler, D. (2015). Observing temporal trends in cardiac rehabilitation from 1996 to 2010 in Ontario: characteristics of referred patients, programme participation and mortality rates. British Medical Journal, 5:e009523. doi:10.1136/bmjopen-2015-009523

Grace, S., Parsons, T., Heise, K., \& Bacon, S. (2015). The Canadian cardiac rehabilitation registry: Inaugural report on the status of cardiac rehabilitation in Canada. Rehabilitation Research and Practice, 2015, doi: 10.1155/2015/278979

Grace, S., Bennett, S., Ardern, C., \& Clark, A. (2014). Cardiac rehabilitation series: Canada. Progress in Cardiovascular Diseases, 56(5), 530-535. doi: 10.1016/j.pcad.2013.09.010 
Grace, S., Leung, Y.W., Reid, R., Oh, P., Wu, G., \& Atler, D. (2012). The role of systematic inpatient cardiac rehabilitation referral in increasing equitable access and utilization. Journal of Cardiopulmonary Rehabilitation, 32(1), 41-47. doi: 10.1097/HCR.0b013e31823be13b

Grace, S., Tan, Y., Marcus, L., Dafoe, W., Simpson, C., Suskin, N., \& Chessex, C. (2012). Perceptions of cardiac rehabilitation patients, specialists and rehabilitation programs regarding cardiac rehabilitation wait times. BMC Health Services Research, 12, 259. doi: $10.1186 / 1472-6963-12-259$

Grace, S.L., Chessex, C., Arthur, H., Chan, S., Cyr, C., Dafoe, W.,...Suskin, N. (2011). Systematizing inpatient referral to cardiac rehabilitation in 2010: Canadian Association of Cardiac Rehabilitation and Canadian Cardiovascular Society joint position paper endorsed by the Cardiac Care Network of Ontario. Canadian Journal of Cardiology, 27(2), 192-199. doi: 10.1016/j.cjca.2010.12.007

Grace, S., Shanmugasegaram, S., Gravely-Witte, S., Brual, J., Suskin, N., \& Stewart, D. (2009). Barriers to cardiac rehabilitation: Does age make a difference? Journal of Cardiopulmonary Rehabilitation and Prevention, 29(3), 183-187.

doi: 10.1097/HCR.0b013e3181a3333c

Gregory, P., LaVeist, T., Simpson, C. (2006). Racial disparities in access to cardiac rehabilitation. American Journal of Physical Medicine \& Rehabilitation, 85(9), 705-710

Grove, S. K., Burns, N., Gray, J. R. (2013). The Practice of Nursing Research: Appraisal, Synthesis, and Generation of Evidence. St. Louis, MI: Elsevier Saunders.

Health Quality Ontario \& Ministry of Health and Long Term Care. (2013). Quality-based 
procedures: Clinical handbook for congestive heart failure. Retrieved from http://www.health.gov.on.ca/en/pro/programs/ecfa/docs/qbp_heart.pdf

Heart and Stroke Foundation. (2016). Heart Failure. Retrieved from, https://www.heartandstroke.ca/heart/conditions/heart-failure

Hutchinson, P., Meyer, A., \& Marshall, B. (2015). Factors influencing outpatient cardiac rehabilitation attendance. Rehabilitation Nursing, 40(6), 360-367. doi: 10.1002/rnj.202

Jackson, M., McKinstry, B., Greogry, S., \& Amos, A. (2012). A qualitative study exploring why people do not attend in cardiac rehabilitation and coronary heart disease self-help groups, and their rehabilitation experience without these resources. Primary Health Care Research \& Development, 13, 30-41. doi:10.1017/S1463423611000284

Johansen, H., Strauss, B., Arnold, J.M., Moe, G., \& Liu, P. (2003). On the rise: The current and projected future burden of congestive heart failure hospitalization in Canada. Canadian Journal of Cardiology, 19(4), 430-435.

Kayaniyil, S., Leung, Y.W., Suskin, N., Stewart, D.E., \& Grace, S.L. (2009). Concordance of self-and program-reported rates of cardiac rehabilitation referral, enrollment and participation. Canadian Journal of Cardiology, 25(4), e96-e99.

Krumholz, H., Merrill, A., Schone, E., Schreiner, G., \& Chen, J., ...Drye, E. (2009).

Patterns of hospital performance in acute myocardial infarction and heart failure 30-day mortality and readmission. Circulation: Cardiovascular Quality and Outcomes, 2, 407413. doi: 10.1161/CIRCOUTCOMES.109.883256 
Lavie, C.J., Thomas, R.J., Squires, R.W., Allision, T.G., \& Milani, R.V. (2009). Exercise training and cardiac rehabilitation in primary and secondary prevention of coronary heart disease. Mayo Clinic Proceedings, 84(4), 373-383. doi: 10.1016/S0025-6196(11)60548X

Lee, D., Johansen, H., Gong, Y., Hall, R., Tu, J., \& Cox, J. (2004). Chapter 14: Regional outcomes of heart failure in Canada. Canadian Journal of Cardiology, 20(6), 599-607. Retrieved from http://www.ccort.ca/Portals/0/atlas/PDF/14_Lee_20_6_04.pdf

Lemstra, M.E., Alsabbagh, W., Rajakumar, R.J., Rogers, M.R., \& Blackburn, D. (2013). Neighbourhood income and cardiac rehabilitation access as determinants of nonattendance and noncompletion. Canadian Journal of Cardiology, 29(12), 1599-1603. doi: 10.1016/j.cjca.2013.08.011

Lewinter, C., Doherty, P., Gale, C.P., Crouch, S., Stirk, L., Lewin, R.J.,...Bland, J.M. (2014). Exercise-based cardiac rehabilitation in patients with heart failure: a metaanalysis of randomized controlled trials between 1999 and 2013. European Journal of Preventative Cardiology. doi:10.1177/2047487314559853

Leon, A.C., Davis, L.L., \& Kraemer, H.C. (2011). The role and interpretation of pilot studies in clinical research. Journal of Psychiatric Research, 45(5), 626-629. . doi: 10.1016/j.jpsychires.2010.10.008

Leung, Y., Grewal, K., Gravely-White, S., Suskin, N, Stewart, D., \& Grace, S. (2011). Quality of life following participation in cardiac rehabilitation programs of longer or shorter than 6 months: Does duration matter? Population Health Management, 14(4). doi: 10.1089/pop. 2010.0048 
Lockwood, E., Hervas-Malo, M., Gutierrez, R., Olson,K., Reddy, M., Shibata, M., Tsuyuki, R. (2004). Patient knowledge of self-care activities in congestive heart failure: the PaKSAC study. Journal of Cardiac Failure, 10(4). doi: http://dx.doi.org/10.1016/j.cardfail.2004.06.421

Mampuya, W. M., (2012). Cardiac rehabilitation past, present and future: an overview. Cardiovascular Diagnosis and Therapy, 2(1), 38-49. doi: 10.3978/j.issn.22233652.2012 .01 .02

Martin, B.J., Hauer, T., Arena, R., Austford, L., Galbraith, D., Lewin, A.,...Aggarwal, S. (2012). Cardiac rehabilitation attendance and outcomes in coronary artery disease patients. Circulation, 126, 677-687. doi: 10.1161/CIRCULATIONAHA.111.066738

McDonall, J., Botti, M., Redley, B., \& Wood, B. (2013). Patient participation in a cardiac rehabilitation program. Journal of Cardiopulmonary Rehabilitation and Prevention, 33, 185-188, doi: 10.1097/HCR.0b013e318282551a

McGrady, A., McGinnish, Badenhop, D., Bentle, M., \& Raiput, M. (2009). Effects of depression and anxiety on adherence to cardiac rehabilitation. Journal of Cardiopulmonary Rehabilitation and Prevention, 29(6), 538-364. doi: 10.1097/HCR.0b013e3181be7a8f

McKee, G., Biddle, M., O’Donnell, Mooney, M., O’Brien, F., \& Moser, D. Cardiac rehabilitation after myocardial infarction: What influences patients'intentions to attend? European Journal of Cardiovascular Nursing,13(14), 329-337. doi: $10.1177 / 1474515113496686$

McMurray, J., Adamopoulos, S., Anker, S., Auricchio, A., Bo“hm, M., Dickstein, K.,... Zeiher, A. (2012). ESC Guidelines for the diagnosis and treatment of acute and chronic heart failure 2012. The Task Force for the Diagnosis and Treatment of Acute and Chronic 
Heart Failure 2012 of the European Society of Cardiology. European Heart Journal, 33, 1787-1847. doi:10.1093/eurheartj/ehs104

Mikkelsen, T., Korsgaard Thomsen, K., \& Tchijevitch, O. (2014). Non-attendance and drop-out in cardiac rehabilitation among patients with ischaemic heart disease.

Danish Medical Journal, 61(10), A4919. Retrieved from

http://www.danmedj.dk/portal/page/portal/danmedj.dk/dmj_forside/PAST_ISSUE/2014/ DMJ_2014_10/A4919

Mochari, H., Lee, J.R., Kligfield, P., \& Mosca, L. (2006). Ethnic differences in barriers and referral to cardiac rehabilitation among women hospitalized with coronary heart disease. Preventive Cardiology, 9(1), 8-13. doi: 10.1111/j.1520-037X.2005.3707.x

Moe, G., Ezekowitz, J., O’Meara, E., Howlett, J., Fremes, S., Al-Hesaven, A.,...White, M. (2013). The 2013 Canadian Cardiovascular Society Heart Failure Management Guidelines Update: Focus on Rehabilitation and Exercise and Surgical Coronary Revascularization. Canadian Journal of Cardiology, 30(3), 249-263. doi: http://dx.doi.org/10.1016/j.cjca.2013.10.010

Moore, C.G., Carter, R.E., Nietert, P.J., \& Stewart, P.W. (2011). Recommendations for planning pilot studies in clinical and translational research. Clinical and Translational Science, 4(5), 332-337. doi: 10.1111/j.1752-8062.2011.00347.x.

Moser, D., Dickson, V., Jaarsma, T., Lee, C., Stromberg, A., \& Riegel, B. (2012). Role of self-care in the patient with heart failure. Current Cardiology Report, 14, 265-265. doi: $10.1007 / \mathrm{s} 11886-012-0267-9$ 
Moser, D.K., Doering, L.V., \& Chung, M.L. (2005). Vulnerabilities of patients recovering from an exacerbation of chronic heart failure. American Heart Journal, 150(5), 984. doi: 10.1016/j.ahj.2005.07.028

Moser, D.K., \& Mann, D.L., (2002). Improving outcomes in heart failure. Circulation, 105, 2810-2812. doi.ord/10.1161/01.CIR.0000021745.45349.BB

Mozaffarian, D., Benjamen, E., Go, A., Arnett, D., Blaha, M., Cushman, M.,...Turner, M. (2015). Heart disease and stroke statistics-2015: a report from the American Heart Association. Circulation, 127, e6-e245. doi: 10.1161/CIR.0000000000000152

Murray, K., Murphy, D., Clements, S.J., Brown, A., \& Connolly, S. (2013). Comparison of uptake and predictors of adherence in primary and secondary prevention of cardiovascular disease in a community-based cardiovascular prevention programme. 36(4), 644-650. doi:10.1093/pubmed/fdt118

Murray, J., Craigs, C., Hill, K., Honey, S., \& House, A. (2012). A systematic review of patient reported factors associated with uptake and completion of cardiovascular lifestyle behaviour change. BMC Cardiovascular Disorders, 12(120). doi: 10.1186/1471-2261-12120

O’Connor, C.M., Whellan, D.J., Kee, K.L., Ketevian, S.J., Cooper, L.S., Ellis, S.J.,...Pina, I.L. (2009). Efficacy and safety of exercise training in patients with chronic heart failure: HF-ACTION randomized controlled trial. JAMA, 301(14), 1429-1450. doi: $10.1001 /$ jama.2009.454

Parajuli, N., Ramprasath, T., Zhabyeyev, P., Patel, V., \& Oudit, G. (2015). The role of neurohumoral activation in cardiac fibrosis and heart failure. In I. Dixon \& J. Wigle (Eds.), Cardiac fibrosis and heart failure: Cause or effect? (pp.347-348). Retrieved from 
https://books.google.ca/books?id=0D0PCgAAQBAJ\&pg=PA347\&lpg=PA347\&dq=caus es + of + hospitalizations + in + Canada + heart + failure \&source $=b l \& o t s=h h O t T 5 U Y d g \& s i g=i B$ E8Q7SffL4D28IqIpjQPPP3x8\&hl=en\&sa=X\&ved=0CD8Q6AEwBWoVChMIrOv61_LtxwIVRzICh3Bbw_z\#v=onepage \&q\&f=false

Parashar, S., Spertus, J.A., Tang, F., Bishop, K.L., Vaccarino, V., Jackson, C.F.,...Sperling, L. (2012). Predictors of early and late enrollment in cardiac rehabilitation, among those referred, after acute myocardial infarction. Circulation, 126(13), 1587-1595.

Pardaens, S., Kerckhove, B., Willems, A., Moerman, V., \& De Sutter, J. (2013). Referral and results of exercise training after hospitalization for heart failure: a comparison with patients after acute coronary syndrome or cardiac syndrome. European Heart Journal, 34(1). doi: 10.1093/eurheartj/eht310.P5785

Paul, S. (2008). Hospital discharge education for patients with heart failure: What really works and what is the evidence? Critical Care Nurse, 28(2), 68=6-82. Retrieved from http://ccn.aacnjournals.org.ezproxy.lib.ryerson.ca/content/28/2/66.full.pdf + html

Pina, I. (2010). Cardiac rehabilitation in heart failure: A brief review and recommendations. Current Cardiology Report, 12, 223-229. doi:10.1007/s11886-010$0100-2$

Piotrowicz, E., Baranowski, R., Bilinska, M., Stepnowska, M., Piotrowska, M., Wo, A.,...Piotrowski, W. (2010). A new model of home-based telemonitored cardiac rehabilitation in patients with heart failure: effectiveness, quality of life, and adherence. European Journal of Heart Failure, 12, 164-171. doi:10.1093/eurjhf/hfp181 dunl 
Phillips, C.O., Wright, S.M., Kern, D.E., Singa, R.M., Shepperd, S, \& Rubin, H.R. (2004). Comprehensive discharge planning with post-discharge support for older patients with congestive heart failure: A meta-analysis. JAMA, 291(11), 1358-1367. Retrieved from

https://www.researchgate.net/publication/8675545_Comprehensive_Discharge_Planning _With_Postdischarge_Support_for_Older_Patients_With_Congestive_Heart_Failure

Public Health Agency of Canada. (2009). Tracking Heart Disease and Stroke in Canada, 2009. Retrieved from http://www.phac-aspc.gc.ca/publicat/2009/cvd-avc/pdf/cvd-avs2009-eng.pdf

Regalbuto, R., Maurer, M., Chapel, D., Mendez, J., \& Snaffer, J. (2014). Joint commission requirements for discharge instructions in patients with heart failure: Is understanding Important for preventing readmission? Journal of Cardiac Failure, 20(9). doi:

10.1016/j.cardfail.2014.06.358

Reges, O., Vilchinsky, N., Leibowitz, M, Khaskia, A., Mosseri, M., \& Kark, J. (2013). Illness cognition as a predictor of exercise habits and participation in cardiac prevention and rehabilitation programs after acute coronary syndrome. BMC Public Health, 13(956). doi: $10.1186 / 1471-2458-13-956$

Ross, H., Howlett, J., Arnold, J.M., Liu, P., O'Neill, B.J., Brophy, J.M... Glasgow K. (2006). Treating the right patient at the right time: Access to heart failure care. Canadian Journal of Cardiology. 22(9), 749-754. Retrieved from http://www.waittimealliance.ca/wpcontent/uploads/2014/05/CCS_Heart_Function_Care.pdf 
Russell, K.L., Holloway, T.M., Brum, M., Caruso, V., Chessex, C., \& Grace, S.L. (2011). Cardiac rehabilitation wait times: effect on enrollment. Journal of Cardiopulmonary Rehabilitation and Prevention. 31(6), 373-377. doi: 10.1097/HCR.0b013e318228a32f

Sanderson, B.K., Shewchuk, R.M., \& Bittner, B. (2010). Cardiac rehabilitation and women: what keeps them away? Journal of Cardiopulmonary Rehabilitation and Prevention, 30(1), 1221. doi: 10.1097/HCR.0b013e3181c85859

Sarrafzadegan, N., Rabiei, K., Shirani, S., Kabir, A., Mohammadifard, N., \& Hamidreza, R. (2007). Drop-out predictors in cardiac rehabilitation programmes and the impact of sex differences among coronary heart disease patients in an Iranian sample: a cohort study. Clinical Rehabilitation, 21, 362-372. Retrieved from http://journals.sagepub.com/doi/pdf/10.1177/0269215507072193

Savage, P., Shaw, A., Miller, M., VanBuren, P., LeWinter, M., Ades, P., \& Toth, M. (2011). Effect of resistance training on physical disability in chronic heart failure. Medicine \& Science in Sports \& Exercise, 43(8), 1379-1386. doi: 10.1249/MSS.0b013e31820eeea1

Shanmugasegaram, S., Oh, P., Reid, R., McCumber, T., \& Grace, S. (2013). Cardiac rehabilitation barriers by rurality and socioeconomic status: a cross-sectional study. International Journal for Equity in Health, doi: 10.1186/1475-9276-12-72

Singa, R., Wright, S., Rubin, H., Phillips, C., Kern, D., \& Shepperd, S. (2004). Comprehensive discharge planning and post-discharge support reduces hospital readmission in older people with congestive heart failure. JAMA, 291(11), 1358-1367. doi: 10.1001/jama.291.11.1358

Smith, E.R., Connolly, C.R., Arthur, H., Boyle, P., Brown, S.,...Campbell, N. (2009). Canadian 
heart health strategy and action plan: Building a healthy heart Canada. Retrieved from http://www.waittimealliance.ca/wp-content/uploads/2014/05/CCS-Building-a-HeartHealthy-Canada.pdf

Smith, K.M., Harkness, K., \& Arthur, H.M. (2006). Predicting cardiac rehabilitation enrollment: the role of automatic physician referral. European Journal of Cardiovascular Prevention and Rehabilitation, 13(1), 60-66. Retrieved from http://journals.sagepub.com/doi/pdf/10.1097/01.hjr.0000186626.06229.7f

Stromberg, A. (2005). The crucial role of patient education in heart failure. The European Journal of Heart Failure, 7, 363-369. doi:10.1016/j.ejheart.2005.01.002

Suskin, N., MacDonald, S., Swabey, T., Arthur, H., Vimr, M.A., \& Tihaliani, R. (2003). Cardiac rehabilitation and secondary prevention services in Ontario: Recommendations from a consensus panel. Canadian Journal of Cardiology, 19(7), 833-888

Taylor, G.H., Wilson, S.L., \& Sharp, J. (2011). Medical, psychological, and sociodemographic factors associated with adherence to cardiac rehabilitation programs: a systematic review. Journal of Cardiovascular Nursing, 26(3), 202-209. doi:

10.1097/JCN.0b013e3181ef6b04

Taylor, R.S., Brown, A., Ebrahim, S., Joliffe, J., Noorani, H., \& Rees, K.,... Oldridge, N. (2004). Exercised-based rehabilitation for patients with coronary heart disease: Systematic review and meta-analysis of randomized controlled trials. American Journal of Medicine, 116(1), 682-692. doi: 10.1016/j.amjmed.2004.01.009

Theou, O., Stathokostas, L., Roland, K.P., Jakobi, J.M., Patterson, C., Vadervoort, A.A., \& Jones, G.R. (2011). The effectiveness of exercise interventions for the management of frailty: a systematic review. Journal of Aging Research, 2011. doi: 10.4061/2011/569194 
Torgrimson, B.N., \& Minson, C.T. (2005). Sex and gender: What is the difference? Journal of Applied Psychology, 99(3), 785-787. doi: 10.1152/japplphysiol.00376.2005

Turk-Adawi, K., Oldridge, N., Tarima, S., Stason, W., \& Shepard, D. (2014). Cardiac rehabilitation enrollment among referred patients. Journal of Cardiopulmonary Rehabilitation and Prevention, 34, 114-122. doi: 10.1097/HCR.0000000000000017

Valenica, H., Savage, P., \& Ades, P. (2011). Cardiac rehabilitation participation in underserved population. Journal of Cardiopulmonary Rehabilitation and Prevention, 31(4), 203-210. doi: 10.1097/HCR.0b013e318220a7da

Weingarten, M., Salz, K., Thomas, R., Squires, R. Rates of enrollment for men and women referred to outpatient cardiac rehabilitation. (2011). Journal of Cardiopulmonary Rehabilitation and Prevention, 31(4), 217-222. doi: 10.1097/HCR.0b013e318207d2fa

Yancy, C.W., Jessup, M., Bozkurt, B., Butler, J., Casey, D.E., Drazner, M.H.,... Wilkoff, B.L. (2013). 2013 ACCF/AHA guideline for the management of heart failure: a report of the American College of Cardiology Foundation/American Heart Association Task Force on practice guidelines. Circulation, 128(16), e240-327. doi:

10.1161/CIR.0b013e31829e8776

Yohannes, A.M., Yalfani, A., Dohert, P., \& Bundy, C. (2007). Predictors of drop-out from an outpatient cardiac rehabilitation programme. Clinical Rehabilitation, 21(3), 229-229

Zhang, L., Sobolev, M., Prince, D., \& Taub, C. (2014). Socioeconomic status, medical insurance and copayment are associated with adherence to cardiac rehabilitation in a multi-racial population. Journal of the American College of Cardiology, 63(12). doi: 10.1016/S07351097(14)61302-4 